\title{
Production, Characterization, and Acceleration of Optical Microbunches
}

\author{
Christopher M.S. Sears
}

SLAC-R-902

Prepared for the Department of Energy

under contract number DE-AC02-76SF00515

Printed in the United States of America. Available from the National Technical Information Service, U.S. Department of Commerce, 5285 Port Royal Road, Springfield, VA 22161. 
This document, and the material and data contained therein, was developed under sponsorship of the United States Government. Neither the United States nor the Department of Energy, nor the Leland Stanford Junior University, nor their employees, nor their respective contractors, subcontractors, or their employees, makes an warranty, express or implied, or assumes any liability of responsibility for accuracy, completeness or usefulness of any information, apparatus, product or process disclosed, or represents that its use will not infringe privately owned rights. Mention of any product, its manufacturer, or suppliers shall not, nor is it intended to, imply approval, disapproval, or fitness of any particular use. A royalty-free, nonexclusive right to use and disseminate same of whatsoever, is expressly reserved to the United States and the University. 


\title{
PRODUCTION, CHARACTERIZATION, AND ACCELERATION OF OPTICAL MICROBUNCHES
}

\author{
A DISSERTATION \\ SUBMITTED TO THE DEPARTMENT OF APPLIED PHYSICS \\ AND THE COMMITTEE ON GRADUATE STUDIES \\ OF STANFORD UNIVERSITY \\ IN PARTIAL FULFILLMENT OF THE REQUIREMENTS \\ FOR THE DEGREE OF \\ DOCTOR OF PHILOSOPHY
}

Christopher M.S. Sears

June 2008 
(C) Copyright by Christopher M.S. Sears 2008

All Rights Reserved 
I certify that I have read this dissertation and that, in my opinion, it is fully adequate in scope and quality as a dissertation for the degree of Doctor of Philosophy.

(Robert H. Siemann) Principal Adviser

I certify that I have read this dissertation and that, in my opinion, it is fully adequate in scope and quality as a dissertation for the degree of Doctor of Philosophy.

(Sami Tantawi)

I certify that I have read this dissertation and that, in my opinion, it is fully adequate in scope and quality as a dissertation for the degree of Doctor of Philosophy.

(Todd Smith)

Approved for the University Committee on Graduate Studies. 


\title{
Production, Characterization, And Acceleration
OF OpticAl Microbunches
}

\author{
Christopher M.S. Sears, Ph.D. \\ Stanford University, 2008
}

\begin{abstract}
Optical microbunches with a spacing of $800 \mathrm{~nm}$ have been produced for laser acceleration research. The microbunches are produced using a inverse Free-ElectronLaser (IFEL) followed by a dispersive chicane. The microbunched electron beam is characterized by coherent optical transition radiation (COTR) with good agreement to the analytic theory for bunch formation. In a second experiment the bunches are accelerated in a second stage to achieve for the first time direct net acceleration of electrons traveling in a vacuum with visible light.

This dissertation presents the theory of microbunch formation and characterization of the microbunches. It also presents the design of the experimental hardware from magnetostatic and particle tracking simulations, to fabrication and measurement of the undulator and chicane magnets. Finally, the dissertation discusses three experiments aimed at demonstrating the IFEL interaction, microbunch production, and the net acceleration of the microbunched beam.

At the close of the dissertation, a separate but related research effort on the tight focusing of electrons for coupling into optical scale, Photonic Bandgap, structures is presented. This includes the design and fabrication of a strong focusing permanent magnet quadrupole triplet and an outline of an initial experiment using the triplet to observe wakefields generated by an electron beam passing through an optical scale accelerator.
\end{abstract}




\section{Acknowledgements}

I would like first to thank Professor Robert Siemann for giving me the opportunity to learn and conduct research these past six years within the Advanced Accelerator Research Department. In my time at SLAC I have learned a great deal, not just the accelerator and laser physics contained here in, but also about the ways and means to conduct research at a large facility like SLAC. Dr. Eric Colby also deserves significant credit for my growth as a graduate student. Dr. Colby's guidance, especially in my first few years as a graduate student, helped me mature quickly as a researcher in this field. I am especially grateful to both Dr. Colby and Professor Siemann for giving me the freedom to guide my own research, determining the make-up of experimental hardware, and the timing and execution of the experiments. I realize the freedom I've had as a graduate student is not often the case on large scale experiments and that I have benefited greatly from it.

In my time at SLAC I have had the pleasure of working alongside a group of intelligent, capable individuals without whose expertise this research could not have been completed. I would like to thank Tomas Plettner, Ben Cowan, Bob Noble, Jim Spencer, Dieter Walz, and Chris McGuinness for all their time, help, wisdom, and support over the years.

Like many large research projects involving particle accelerators, ours requires and benefits from the support of many individuals with a range of expertise. Although the full range of individuals who contributed to the research would require many pages, there are several to whom I owe a special thanks. I would like to thank Roger Carr, Denise Larsen, and Zack Wolf for all there help and advice during the design, fabrication, and measurement of the magnetic hardware. Mike Hennessy at 
Stanford University and Mike Racine gave excellent support for which I am grateful in the installation of the experiments at HEPL and at SLAC. Finally, I would like to thank the NLCTA operations group: Janice Nelson, Doug McCormick, Tonee Smith, Keith Jobe, and Justin May - for contributing their time and expertise in running the accelerator.

Beyond the immediate influences on my career of those at SLAC and Stanford, I owe a great deal of gratitude to many people I have known during my life, from undergraduate professors and grade school teachers, to my friends and family. These influences have defined the person I am today. In May of 2006 I met my new wife Laura, whose love and support these past few years has given me the strength to finish my graduate career.

As I reflect back on the influences that come to bear on my completion of a $\mathrm{PhD}$ program, one stands out more than any other. That is, that of my father, Derek Sears. From childhood projects; before dinner discussions of physics, chemistry, history; to counsel on research group social dynamics, my father has always been there for me as an advisor, mentor and role-model. I dedicate this dissertation to him. Thank you, father. 


\section{Contents}

Acknowledgements vii

1 Introduction 1

1.1 A Case for Laser Acceleration . . . . . . . . . . . . . . . . . . . . 1

1.2 Prior Experiments in Laser Acceleration . . . . . . . . . . . . . . . . 4

1.3 The Laser Electron $\underline{\text { Acceleration }}$ Project at Stanford . . . . . . . . . 6 1.3.1 The ITR Accelerator . . . . . . . . . . . . . . . . . 7

1.4 The Need for Optical Microbunching and the E163 Program . . . . . 9

2 FEL Theory \& Microbunching 13

2.1 IFEL Physics . . . . . . . . . . . . . . . . . . . . . . . . . . 14

2.2 IFEL Interaction Bandwidth . . . . . . . . . . . . . . . . 17

2.3 Higher Harmonic Interactions . . . . . . . . . . . . . . . . . . . . . . 19

2.4 Microbunching in a Chicane . . . . . . . . . . . . . . . 21

2.4.1 Microbunch Washout . . . . . . . . . . . . . . . . . 23

2.5 Simulation of Microbunching Experiment . . . . . . . . . . . 25

3 Hardware Design and Fabrication $\quad 27$

3.1 Field Integrals of Magnets _ . . . . . . . . . . . . . . . . . 27

3.2 Design Considerations for LEAP/E-163 . . . . . . . . . . . . 30

3.3 Simulation Software: Radia and Mafia . . . . . . . . . . . . . . . 32

3.3.1 Particle Tracking through Fields . . . . . . . . . . . . . . . . . 33

3.4 Design Results . . . . . . . . . . . . . . . . . . . . . . 34

3.4.1 The Undulator . . . . . . . . . . . . . . . . . . 34 
3.4 .2 The Chicane . . . . . . . . . . . . . . . . 36

3.5 CAD Design, Fabrication, and Testing $\ldots \ldots \ldots \ldots$

3.5.1 The Undulator Error and Correction _ . . . . . . . . . 40

4 The Higher Harmonics Experiment 43

4.1 Motivation for and description of the experiment . . . . . . . . 43

4.2 Experiment Results . . . . . . . . . . . . . . . . . . . . 46

4.3 Implications for Advanced Accelerator Research . . . . . . . . . . 50

5 The E163/NLCTA Facility 53

5.1 The Laser Room . . . . . . . . . . . . . . . . . . . . . 54

5.2 The NLCTA beamline . . . . . . . . . . . . . . . . . 57

5.2 .1 Beam Characterization . . . . . . . . . . . . . . 62

5.3 The E163 Experimental Hall . . . . . . . . . . . . . . . . . . . . 64

$5.3 .1 \quad$ Laser/Electron Alignment . . . . . . . . . . . . . . . 66

6 Microbunch Formation and Characterization $\quad 69$

6.1 Microbunch Characterization using Transition Radiation . . . . . . 70

6.2 Experiment Layout and Methodology . . . . . . . . . . . . 74

6.3 Results from the Experiment . . . . . . . . . . . . . . . . 79

7 Net Acceleration of Electrons with Light $\quad 83$

7.1 The Second Stage of the Net Acceleration Experiment . . . . . . . 84

7.2 Expected Signals and Monte Carlo Simulations _ . . . . . . . 86

7.3 Experiment Layout . . . . . . . . . . . . . . . . . . . . . . . 89

$7.3 .1 \quad$ IFEL-ITR Phase Stability . . . . . . . . . . . . . . . 92

7.4 Results . . . . . . . . . . . . . . . . . . . . . . . 94

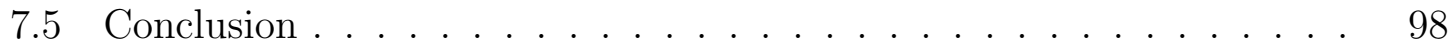

8 Tight Focusing of Electron Microbunches $\quad 99$

8.1 Optical Scale Structures And Tight Focusing . . . . . . . . . . . . 99

8.2 Beam Transport and Focusing . . . . . . . . . . . . . . . . 101

8.2.1 Washout of a Tightly Focused Microbunched Beam . . . . . 102 
8.3 Designing the Permanent Magnet Quadrupole Triplet . . . . . . . . . 104 8.3.1 PMQ Measurement . . . . . . . . . . . . 107

8.4 Optical Wakefields From a Photonic Bandgap Fiber . . . . . . . . . . 114

8.4.1 Resonant Cherenkov Wakefield from a Bragg Fiber . . . . . . 117

8.4.2 The 3-D case: Crystal Fibre HC-1550-02 Fiber[1] . . . . . . . 119

8.4.3 Potential Experimental Arrangement . . . . . . . . . . . . . . 119

9 Conclusion $\quad 123$

9.1 Future of the E163 Program . . . . . . . . . . . . . . . . 124

9.2 Potential Impact on the Broader Research Community . . . . . . . . 127

A Multipole Expansion of Magnetostatic Fields

B Bootstrap Error Analysis 131

Bibliography

133 


\section{List of Tables}

2.1 E163 Experimental Parameters. . . . . . . . . . . . . . . . . 14

3.1 Undulator design specifications . . . . . . . . . . . . . 34

3.2 Chicane design specifications . . . . . . . . . . . . . . 38

4.1 Experiment Parameters . . . . . . . . . . . . . 46

5.1 E163 Laser specifications . . . . . . . . . . . . . . . 56

5.2 NLCTA parameters . . . . . . . . . . . . . . 61

6.1 Parameters of Microbunching Experiment . . . . . . . . . . 77

8.1 PMQ design specifications . . . . . . . . . . . . . . 106

$8.2 \mathrm{PMQ}$ decopole terms . . . . . . . . . . . . . . . . 113 


\section{List of Figures}

1.1 The LEAP cell . . . . . . . . . . . . . . . . . . . . . . . . . . 7

1.2 ITR Results . . . . . . . . . . . . . . . . . . . . . . 8

2.1 Diagram of a Free Electron Laser . . . . . . . . . . . . . . 15

2.2 Microbunching Density . . . . . . . . . . . . . . . . . . . . . . 22

2.3 Microbunch washout due to divergence . . . . . . . . . . . . . . 24

3.1 The $2^{\text {nd }}$ Field Integral $\ldots \ldots \ldots \ldots \ldots \ldots \ldots$

3.2 Radia CAD of the undulator . . . . . . . . . . . . . . . . 35

3.3 Radia CAD of Chicane . . . . . . . . . . . . . . . . . 37

$3.4 \quad$ H-magnet Field Profiles . . . . . . . . . . . . . . . . . . . . 38

3.5 Undulator Design Progression . . . . . . . . . . . . . . . . . 40

3.6 Chicane after Fabrication . . . . . . . . . . . . . . . . . . . . 41

3.7 The Undulator Field Error . . . . . . . . . . . . . . . . . . . . . . 41

4.1 IFEL Harmonic Coupling Coefficients . . . . . . . . . . . . . . . 44

4.2 E-beam trajectory in undulator . . . . . . . . . . . . . 47

4.3 Example Data Run . . . . . . . . . . . . . . . . . . . . . . . . 48

4.4 Higher Harmonic Experiment Transverse Overlaps . . . . . . . . . . . 49

4.5 Higher Harmonic Experiment Gap Scan Data . . . . . . . . . . . 51

5.1 Layout of E163 laser system. . . . . . . . . . . . . . . . . . 54

5.2 Layout of the pulse front tilt. The red lines indicate the progression of the pulse front tilt. . . . . . . . . . . . . . . . . 59

$5.3 \quad$ NLCTA/E163 diagram . . . . . . . . . . . . . . . . . . 60 
5.4 Quad Scans . . . . . . . . . . . . . . . . . . . . . . . 63

5.5 NLCTA Dispersion Scan . . . . . . . . . . . . . . . . . . 64

6.1 COTR angular distribution $\ldots \ldots \ldots \ldots \ldots \ldots$

$6.2 \quad$ COTR yield . . . . . . . . . . . . . . . . . . . . . . . 73

6.3 Microbunch Experiment Layout . . . . . . . . . . . . . 75

6.4 Example run for COTR Experiment . . . . . . . . . . . . 78

6.5 COTR Yield versus IFEL Modulation . . . . . . . . . . . . . 79

6.6 COTR yield versus chicane strength . . . . . . . . . . . . . . . 81

6.7 Drifts during chicane scan $\ldots \ldots \ldots \ldots$

7.1 Illustration of ITR-microbunch Horizontal Phase . . . . . . . . . 85

7.2 Evolution of a microbunch in the net acceleration experiment. . . . . 87

7.3 Monte Carlo of the net acceleration experiment . . . . . . . . . 88

7.4 Net acceleration experiment layout . . . . . . . . . . . . . . . . 89

7.5 Phase Monitor Layout . . . . . . . . . . . . . . . . . . . . 92

7.6 Phase Stability of the Net Acceleration Experiment . . . . . . . . . 94

7.7 Net Acceleration Signal in the Frequency Domain . . . . . . . . 96

7.8 Net acceleration correlation plots . . . . . . . . . . . . . . 97

8.1 Focusing of Two Triplets . . . . . . . . . . . . . . . . . . . . . 102

8.2 Beam Transmission through Optical Scale Structures _. . . . . . . . 103

8.3 Longitudinal Slippage in the PMQ triplet . . . . . . . . . . . . . . 104

8.4 Microbunch attenuation factors due to focusing in the PMQ triplet . 105

8.5 PMQ Comparison . . . . . . . . . . . . . . . . . . . . . 106

8.6 The Permanent Magnet Quadrupole Triplet . . . . . . . . . . . . 107

8.7 Hall probe scans of PMQ triplet . . . . . . . . . . . . . . . . 108

8.8 The pulsed wire scan setup . . . . . . . . . . . . . . . . . . . 109

8.9 Wire scan dispersion compensation . . . . . . . . . . . . . 111

8.10 Image of coil tip . . . . . . . . . . . . . . . . . . . . . . . 112

8.11 PMQ rotating coil spectrum . . . . . . . . . . . . . . . . 113

8.12 PMQ focusing properties with errors . . . . . . . . . . . . 114 
8.13 Accelerating mode in a Photonic Bandgap Fiber . . . . . . . . . . . . 116

8.14 Wakefield simulation of a Bragg fiber . . . . . . . . . . . . . . 117

8.15 Wakefield simulation of Thorlabs HC-1550 fiber . . . . . . . . . . . . 120

8.16 PBG Fiber wakefield experiment layout . . . . . . . . . . . . . . . 121

9.1 Potential Optical Scale Accelerator Structures . . . . . . . . . . . 126 
xviii 


\section{Chapter 1}

\section{Introduction}

\subsection{A Case for Laser Acceleration}

Like many devices, the history and development of particle accelerators have been marked by the introduction of new technologies that advance the field in leaps rather than by a gradual improvement of pre-established technologies over time. The first particle accelerators[2] relied on electrostatic fields much like the Van der Graff generator producing beams of a few $\mathrm{MeV}$ limited by dielectric breakdown of isolating insulator components. The introduction of alternating gradient technology[3] allowed beam energies beyond this limit. A progression of new technologies has eventually led to modern day RF electron accelerators that have acceleration gradients of a few tens of megavolts per meter producing beam energies of tens of $\mathrm{GeV}$ in several kilometer long accelerators. These accelerators have been used to great success in increasing humanity's understanding of fundamental physics at the subatomic scale. Future plans from the high energy physics community seek to probe among other things the nature of gravity and matter by going to still higher center-of-mass energies, upwards of $1 \mathrm{TeV}$. With the current state of the art technology in order to reach this desired center-of-mass energy the accelerator would need to be tens of kilometers in length[4]. Increasing the energy still further would likely be prevented by excessive

civil construction costs and power requirements, and potentially even shortages of raw materials needed to construct the accelerator cells. To avoid this, the machine 
must be made smaller by increasing the accelerating gradient. Unfortunately current technologies are already at their limit in sustaining high electric fields. However, at optical frequencies the ability to create and confine strong electromagnetic fields is much better. With the development of chirped pulse amplification (CPA)[5] in the mid 1980s lasers are readily capable of producing electric fields in excess of $100 \mathrm{GV} / \mathrm{m}$ in pulses less than 1 ps duration. Even more recently, development of diode pumping and low quantum defect materials has led to dramatic increases in total efficiency of laser systems. These laser sources and their continued development make attractive the prospect of accelerating electrons using lasers.

The current mainstream accelerator technology is the disc-loaded RF waveguide. The waveguide is formed from a set of individual copper discs each with a central aperture that are stacked together to form a waveguide. The space between two adjoining discs forms a cavity which resonates at radio or microwave frequencies. The input coupling and cavity geometry are arranged such that the resonant mode has a longitudinal accelerating electric field. The irises serve both as the aperture for the electron beam and as a means for RF field to couple from one cavity to the other. At each end of a stack is attached a modified resonator cell with an additional aperture transverse to the disc that allows input/output coupling of the RF fields. Since the accelerator is in effect a set of resonators with a Q-factor much greater than 1, a long RF pulse is needed to build up the necessary field intensity (typically $\sim 1 \mu s)$.

Current accelerator field strengths are limited by two processes due to the strong RF fields inside the structures: pulsed heating and RF breakdown [6]. The pulsed heating from high power RF causes mechanical stresses in the accelerator structure leading to micro-crack formation, or cavity deformation and de-tuning. RF breakdown on the other hand is a single shot failure in which electrons are emitted from a surface within the accelerator, accelerated by the RF fields, and impact upon another surface within the accelerator. The number of electrons and ions can build up much like in a photomultiplier eventually damaging the accelerator. The physics of pulsed heating and RF breakdown remain an area of active research, though these effects currently limit accelerators to unloaded gradients of $\sim 90 \mathrm{MV} / \mathrm{m}$ with a maximum of 
$140 \mathrm{MV} / \mathrm{m}$ demonstrated in a single test structure[7]. For most linear accelerators currently in operation, the gradient is typically $\sim 30 \mathrm{MV} / \mathrm{m}$.

Optical accelerators manage the damage issue in a couple of different ways. Some acceleration schemes studied so far accelerate using a free-space laser mode, eliminating a electromagnetic guiding structure and thus any issue with its damage. However, in a purely free space interaction between a laser and an electron, to first order in the fields the net energy gain is zero because of the inevitable phase slip between the laser with phase velocity $v_{p h}=c$ and the electron with $v=\beta * c$. This was first noted by J.G. Woodward and J.D. Lawson[8], and is often referred to as the Lawson-Woodward theorem. Acceleration can still be achieved from free space traveling lasers by instead matching the optical and electron phase velocity either via an undulator in the inverse free electron laser (IFEL) experiments $[9,10,11]$ or with a low pressure gas in inverse Cherenkov acceleration $[12,13]$ or by relying on a higher order interaction between the laser and electron (ponderomotive acceleration) which requires very high laser intensity[14]. These experiments however all have the significant disadvantage that the coupling efficiency between laser and electron is very low with the vast majority of the laser pulse energy wasted. To achieve reasonable efficiency, a guiding structure is still necessary. Early proposals simply scaled down existing metallic structures to laser wavelengths[15]. However, at optical frequencies absorption in metals becomes significant. Optical structures would therefore be made of dielectric materials. Dielectric materials also have a much higher damage threshold than metals. For example a $1 \mathrm{ps}, 800 \mathrm{~nm}$ pulse the multi-shot damage threshold of fused silica has been measured to be an incident fluence of $2-4 \mathrm{~J} / \mathrm{cm}^{2}[16]$ compared to 0.1-0.2 J/ $\mathrm{cm}^{2}$ for gold[17]. This translates to a maximum surface field of $3.9 \mathrm{GV} / \mathrm{m}$ giving over an order of magnitude improvement compared to RF. Less well known is the limit due to pulsed heating; however given the very short pulses $(\sim 1 \mathrm{ps})$ and low pulse energies $(<100 \mu \mathrm{J})$ heating should not be a factor in low repetition rate accelerators. For high repetition rates discussed in [18] average heating may become an issue.

Besides the considerable benefit of increased allowable peak surface field, optical accelerators have additional benefits in sharing technology and discovery with 
a large and active research field driven in large part by the telecommunications industry. Photonic bandgap fibers are being pursued for their long-haul, low loss as well as customizable dispersion characteristics, but they may also have applications to laser acceleration as optical vacuum waveguides. Similarly, lithographically made photonic bandgap devices are being studied for all-optical switches and couplers, research which could be adapted to create an accelerator-on-a-chip. Meanwhile, rapid progress is being made in high power, high efficiency mode locked lasers which have reached optical-to-optical efficiencies of $\sim 50 \%[19]$, competitive with the most efficient klystrons used in standard accelerators.

The wealth of research and application of optical devices, both for efficiently producing light and guiding in optical scale structures, could potentially lead to compact, inexpensive electron sources. By applying the manufacturing processes involved in making semiconductor computer chips to accelerator research, one might one-day produce a monolithic accelerator-on-a-chip, complete with power source[20], electron emitter, and accelerator structure.

With these benefits in mind, we seek to demonstrate as a first step optical acceleration of electrons traveling in a vacuum. As with many research endeavors, achieving the final goal of an accelerator-on-a-chip in a single step would be effectively impossible in a single project. Besides the optical accelerator, a full system will require the power source, electron emitter, waveguides, magnets, and a host of other components. Several of these are being actively studied by other researchers. It is the goal of this work to first demonstrate optical acceleration.

\subsection{Prior Experiments in Laser Acceleration}

The idea of using lasers to accelerate particles was first proposed by Shimoda [21] and others in the 1960's, while lasers themselves were still in their infancy. It was not until much later that the first experiments demonstrating acceleration of electrons with light appeared. The first experiment to interact electrons directly with a laser used the inverse of the Cherenkov radiation process[12] (ICA). The initial energy modulation was small, just $37 \mathrm{keV}$ over a $100 \mathrm{~cm}$ helium filled gas cell, due largely to the laser's 
small peak fluence. Later experiments [13] replaced the Q-switched Nd:YAG laser with a sliced $\mathrm{CO}_{2}$ laser to obtain a much higher peak fluence and therefore a stronger interaction. A radial polarizer was also used to better match the incoming laser to the Cherenkov radiation pattern. The acceleration mechanism in ICA is a direct interaction between the laser field and the electrons using the gas to phase match. However, the gas scatters the electron beam and leads to emittance growth, limiting the usefulness of ICA for longer accelerators. This cannot be circumvented with stronger laser fluences as the gas would breakdown under large laser fields leading to strong absorption and electron-plasma interactions.

Later experiments aimed to achieve direct laser acceleration of electrons in a vacuum. Two separate experiments used the inverse of the Free Electron Laser process (IFEL) to both bunch and accelerate electrons $[9,10,11]$. Both the experiments used large, complex $\mathrm{CO}_{2}$ lasers for the driver. In the STELLA experiment[9], the laser pulse was split between two undulators. In the first undulator, a relatively small interaction sinusoidally modulated the electron energy as a function of electron longitudinal position with the bunch. A chicane after the first stage was used to convert the energy modulation into a density modulation forming a train of microbunches. In the second undulator, the microbunches were then accelerated. Although these experiments together represent a significant contribution to advanced accelerator research, demonstrating significant acceleration gradient over a long $(\sim 1 \mathrm{~m})$ distance in vacuum, they did not achieve much reproducibility due in large part to significant spatial and energy jitter of the driving lasers. $\mathrm{CO}_{2}$ lasers are a mature technology having reached wall plug efficiencies of around $20 \%$ which, though good, does not reach the efficiency of current conventional accelerator drivers nor the efficiency of solid state and fiber lasers. There is also relatively little interest in $\mathrm{CO}_{2}$ lasers in the broader academic and technical community making the chance for collaboration or technology sharing less likely. In addition, while the IFEL process has many advantages including relatively easy optical coupling and alignment compared to optical scale structures, the IFEL process is not scalable to high energies due to roll-off in interaction strength and the scaling of the undulator needed to maintain resonance.

In parallel to the efforts to accelerate electrons directly with a laser field, other 
research projects proposed and performed acceleration indirectly by using a wakefield in a plasma[22]. In such experiments, a tightly focused intense laser pulse (fluence $>10^{18} \mathrm{~W} / \mathrm{cm}^{2}$ ) propagates through a plasma expelling plasma electrons due to a ponderomotive force. After the laser pulse the plasma electrons rush back setting up a wakefield in which an electron beam can be trapped and accelerated. This line of research has grown into a large and successful field, recently demonstrating trapping and acceleration of a mono-energetic electron beam[23]. Other experiments used a similar physical mechanism by shining a laser onto a thin foil to ablatively accelerate particles, both electrons and ions, from the surface[24]. Further discussion of these acceleration schemes is outside the scope of this thesis.

\subsection{The Laser Electron Acceleration Project at Stanford}

The Laser Electron Acceleration Project (LEAP experiment) began almost a decade ago at Stanford University as an effort to achieve a first order laser-electron interaction in vacuum. In its beginnings LEAP sought to accelerate electrons in vacuum using a laser field confined by a dielectric structure surrounding the beam. Thus it had a modest laser system ( $1 \mathrm{~mJ} /$ pulse) since larger pulse energies would damage the dielectric. The project initially used a geometry with two crossed laser beams[25] with the electrons traveling in a semi-open space between a pair of reflectors (figure 1.1). Each beam was at an angle providing partial electric field in the longitudinal direction while the transverse components of the fields canceled on-axis providing net acceleration with no transverse kick. Since the laser travels at an angle $\alpha$ relative to the electron trajectory, the electrons see phase fronts traveling at:

$$
v_{p h}=\frac{c}{\cos \alpha}
$$

For a crossing angle of $\alpha=34 \mathrm{mrad}$ the electrons will therefore slip relative to the laser by $\lambda / 2$ after about $1 \mathrm{~mm}$. This slippage distance sets the gap between the two pairs of mirrors. The upstream set of mirrors are also angled by $45^{\circ}$ to bring the laser 
beams in and out of the interaction region.

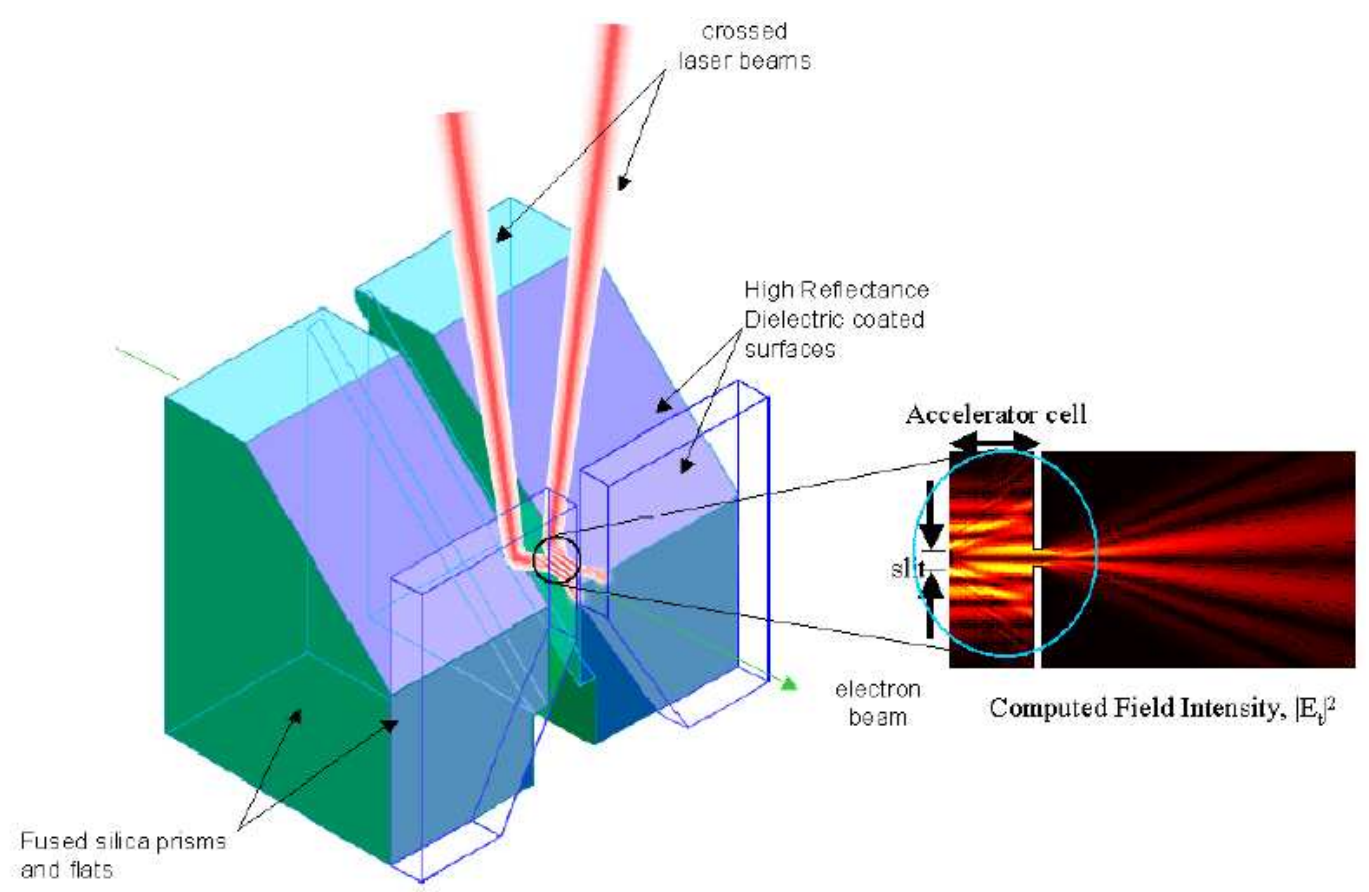

Figure 1.1: The LEAP cell.

\subsubsection{The ITR Accelerator}

Unfortunately LEAP ran into difficulties in its early years owing to considerable experimental challenges and a lack of accelerator time. However, in August of 2004 the experiment succeeded in its goal of interacting 800nm light via an inverse transition radiation (ITR) process[26]. The same experimental run also allowed for testing of an IFEL process at higher harmonics[27]. The IFEL harmonics experiment is described in detail in chapter 4.

The ITR experiment started as a simplification of the initial LEAP structure[25]. The first simplification eliminated the upstream boundary and brought the laser essentially from infinity terminating on a single metallic surface. The second simplification did away with a second laser beam. In the original LEAP structure two beams 

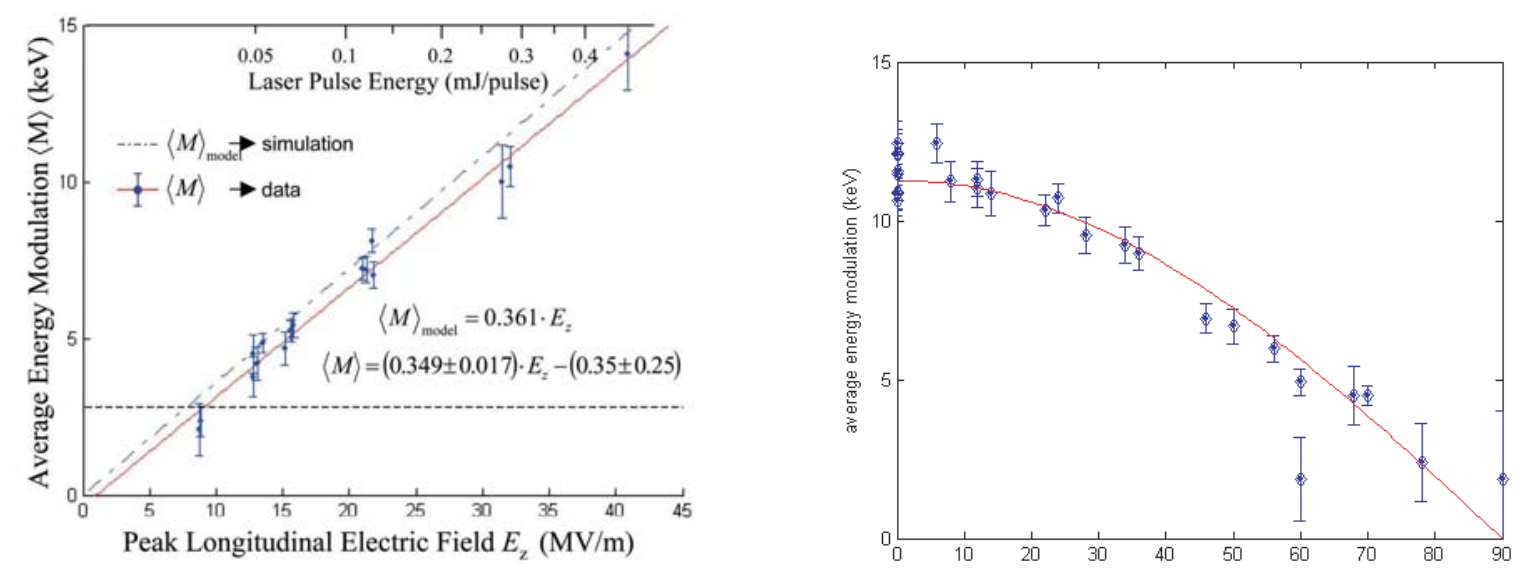

Figure 1.2: Results of the Inverse Transition Radiation Experiment. Left: Plot of electron energy modulation versus laser pulse energy. The interaction is shown to be linear in the electric field. Right: electron energy modulation versus laser polarization angle. Both scans show excellent agreement with theory. Figure reproduced from [26].

are combined such that the transverse fields cancel while the longitudinal accelerating fields add. This simplification would seem to introduce a small transverse kick, however as noted in [28], for an ultra-relativistic beam and a crossing angle $\alpha=1 / \gamma$ there is no kick due to a cancellation between the electric and magnetic Lorentz forces. A third modification that led to the ultimate success of the experiment was the introduction of a tape system to advance the metal foil to a new section between shots. This allowed the laser power to be increased beyond the damage threshold of the boundary allowing for greater laser-electron interaction. Although the surface ultimately damages, during the short time the electrons and laser are present at the tape surface, the tape remains an optically flat surface. This was verified before the experiment by standard pump-probe measurements.

The ITR experiment succeeded in showing, for the first time, a direct, linear acceleration of electrons using visible light. The overall interaction strength was quite modest, just 30-40 keV fwhm. To show that the interaction was due to the inverse transition process, a couple of different scans were done varying the laser intensity and polarization (figure 1.2). The interaction was proven to be linear in the electric field and polarization dependent. The polarization dependence eliminated a possible indirect mechanism due to plasma formation at the boundary. The data agree very 
well with theory.

One can define an acceleration gradient as the coherence length of the ITR interaction $L_{c}$ divided by the acceleration energy. The maximum accelerated particles changed energy by $\sim 20 \mathrm{keV}$ (half the fwhm). The coherence length is the distance over which a single electron remains within a single half-cycle of the laser field. This is given by $L_{c}=0.5 \lambda_{l} \gamma^{2}=1.4 \mathrm{~mm}$. Then the average gradient $G$ is $G=20 \mathrm{keV} / L_{c}=14$ $\mathrm{MeV} / \mathrm{m}$. Thus, for the range of parameters used in this experiment, the acceleration gradient is quite similar to a traditional accelerator.

\subsection{The Need for Optical Microbunching and the E163 Program}

In these initial demonstration experiments at LEAP, both the laser and electron beams were $1 \mathrm{ps}$ in duration. By comparison, the optical period of the laser was just $2.67 \mathrm{fs}$. As a result, there are electron-laser interactions at all phases resulting in an increase in energy spread rather than net acceleration. Indeed, the results of the initial LEAP experiments are all expressed as the increase in energy spread. However, an important stated goal of LEAP was to obtain net acceleration. This could in theory be obtained by starting with sub-fs electron pulses. However this is currently well beyond the abilities of conventional particle accelerators and all-optical injectors do not yet exist. Instead, the picosecond long electron bunch available must be sub-divided, or microbunched, to match the accelerating wavelength.

Shortly after completing the initial demonstration of optical acceleration the research project was moved to a new facility at SLAC. The project was assigned a SLAC experiment number: E163. One of the first goals of the research program at its new locale was the production and characterization of optical microbunches for use in laser acceleration research. This research is the focus of this dissertation.

Optical microbunching is not a new technique. Optical microbunches were first created as a necessary step in Free Electron Laser[29] and Optical Klystron[30] development and operation in the 1970s. These devices used the microbunching process 
to achieve high-gain coherent emission at optical frequencies from electron motion in undulating magnetic fields. In some sense these devices could be considered as an extension of klystrons[31] to relativistic beam energies. One significant difference in the optical devices compared to the klystron though was the lack of a small seed source for the electromagnetic field. Instead, both FELs and optical klystrons start from Schottky noise in the beam itself.

Much like the optical klystrons created for synchrotrons, the E163 program will use a short undulator magnet followed by a dispersive chicane magnet to create optical microbunches. Instead of building up from noise though, the undulator is seeded by a strong laser field with the same wavelength as the acceleration section to follow. This allows for phase synchronicity between the microbunches and the accelerator. It also makes the modulator section much shorter than in an optical klystron. Although the physics remains the same, because of the strong optical field at the start of the undulator and the key interest being the microbunching rather than the radiation, this process is termed an Inverse Free Electron Laser interaction.

Chapter 2 goes over basic FEL theory needed for understanding the IFEL process and resulting energy modulation. The chapter also discusses production of optical microbunches and the characteristics of those bunches. Chapter 3 discusses the design of the undulator and the dispersive chicane including constraints from the experiment, magnetostatic \& CAD designs, and measurement after assembly. Chapter 4 discusses the first experiment using the IFEL alone which explored harmonics of the IFEL interaction. This experiment was done ahead of completion of the E163 facility for laser acceleration research. Chapter 5 gives an overview of the newly completed E163 facility for laser acceleration research. Chapters 6 and 7 present the main experimental effort of this thesis in first producing and characterizing optical microbunches (chap. 6) and then obtaining net acceleration in a staged experiment (chap. 7).

The eighth chapter of this thesis presents an entirely different challenge presented by direct laser acceleration. Laser acceleration schemes presented thus far involve largely free space propagation of the laser with transverse dimensions much larger than the accelerating wavelength. The eighth chapter introduces laser accelerators with dimensions at the optical scale, and discusses the steps necessary to focus the 
electron beam to such small transverse dimensions. This includes an explanation of tight focusing of electrons, as well as the design and fabrication of a strong quadrupole triplet needed to produce the focus. Finally, the chapter ends with an outline of the initial experiments planned using the tight focus which will explore wakefields produced from optical scale accelerators. 


\section{Chapter 2}

\section{FEL Theory \& Microbunching}

Free Electron Lasers (FEL) are at this point well developed and understood devices. An FEL relies on radiation from a 'free' electron as opposed to electrons bound in atomic states. Typically, the term FEL refers to the production of radiation from an electron passing through an undulating magnetic field, though strictly speaking the radiating process can be one of a number of processes including Cherenkov radiation or the Smith-Purcell effect. For the purposes of this thesis, the term FEL will refer to undulator radiation based devices. The first FEL produced was a device called the 'ubitron' which used a non-relativistic high current beam to produce microwave power with $10 \%$ efficiency [32]. However, cavity based klystrons and gyrotrons were already producing similar wavelength power at much greater efficiency, and the technology was not pursued further until the mid 70's when an FEL was installed with a 24 $\mathrm{MeV}$ beam to produce mid-infrared radiation [33]. FEL development then progressed quickly, and FELs are now found throughout the world.

The theory of FELs is well developed. A good reference used for much of the theoretical backing of this thesis is Charles Brau's book [34]. The section below goes over only the small portion of theory needed to understand the inverse FEL interaction. Section 2.3 goes into further detail to describe coupling of electrons to higher harmonics in an undulator. Section 2.4 describes microbunching formation within the chicane and discusses sources of microbunch washout. Section 2.5 introduces the simulation methods used throughout the thesis. 
Although the E163 program and the magnetic hardware are described in thorough detail in later chapters, it is useful to have a few important parameters listed in this chapter for reference. Table 2.1 gives some of the experimental parameters.

\begin{tabular}{|l|l|c|}
\hline & Parameter & Value \\
\hline The Beam & & \\
& Energy & $60 \mathrm{MeV}$ \\
& Energy Spread & $0.1 \%$ \\
& Charge & $50 \mathrm{pC}$ \\
The Undulator & & \\
& & \\
& Period & $1.8 \mathrm{~cm}$ \\
& Number of Periods & 3 \\
& $K_{w}$ & $0.7-1.2$ \\
\hline
\end{tabular}

Table 2.1: E163 Experimental Parameters.

Figure 2.1 shows a diagram of an FEL. The alternating vertical magnetic field causes the electron beam to oscillate in the horizontal plane. The electrons radiate due to the transverse acceleration. The radiation cone overlaps with that of prior periods leading to coherence at certain frequencies as subsequent lasing. In the inverse case, the process begins with a strong radiation field in the form of a laser pulse already present at the start of the undulator. The laser polarization is in the same direction as the electron motion in the undulator. The laser propagates at the speed of light while the electrons are slowed due to their transverse motion in the undulator. The undulator period is chosen such that for each oscillation in the undulator field, the electron slips relative to the laser phase by one optical cycle. This condition, known as the FEL resonance condition, is the same criteria that leads to coherence at a single frequency in the forward, FEL, case.

\subsection{IFEL Physics}

The physics necessary to describe and understand an IFEL are much simpler than the forward, FEL, case. Given the large initial field, any changes to the field from the electrons are very small, and emission/absorption can be ignored. Also, the 


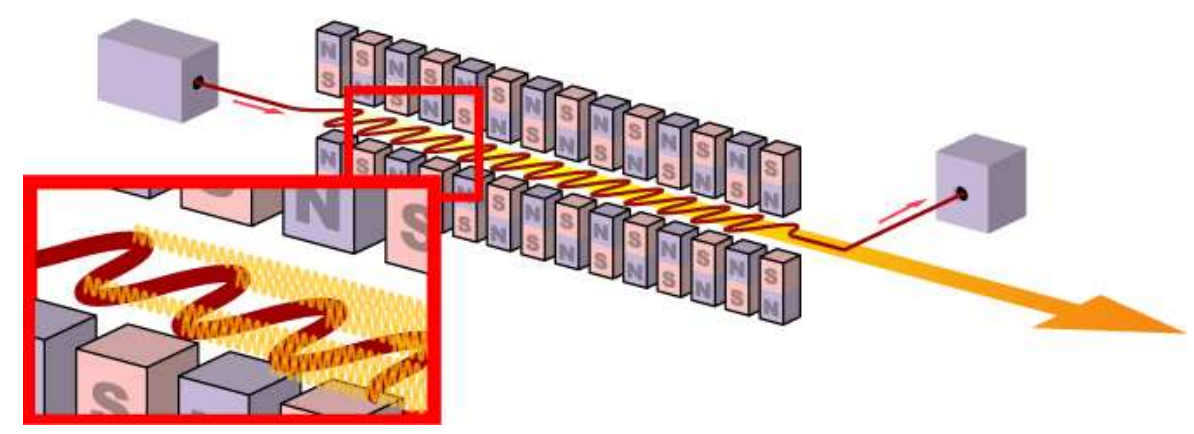

Figure 2.1: Diagram of a Free Electron Laser showing the electron oscillation and subsequent radiation.

short length of the undulator means that very little phase slippage within the bunch can occur; all the bunching occurs within a downstream chicane. The energy gain and motion of the electrons can be understood purely from examining the Lorentz equations of the electron in the combined undulator and laser fields. To illustrate, we approximate the laser by a plane wave polarized in the direction of oscillation of the electrons, and ignore roll-off and gap variation of the undulator field. Under these simplifications we have the following field components:

$$
\begin{array}{r}
\vec{E}_{\text {laser }}=E_{l} \hat{x} \sin \left[k_{l}(z-c t)\right] \\
\vec{B}_{\text {laser }}=\left(\frac{E_{l}}{c}\right) \hat{y} \sin \left[k_{l}(z-c t)\right] \\
\vec{B}_{w i g}=B_{w} \hat{y} \cos \left(k_{w} z\right)
\end{array}
$$

Here, $k_{l}=2 \pi / \lambda_{l}$ is the laser wavenumber and $k_{w}=2 \pi / \lambda_{w}$ is the undulator wavenumber; $\lambda_{w}$ is the undulator period. Putting these into the Lorentz force equations gives the motion of the electron in the IFEL. Assuming the electron initially has $\dot{v}_{x}=\dot{v}_{y}=0$ we have:

$$
\frac{d v_{x}}{d t}=\frac{q}{\gamma m_{e}}\left(E_{l} \sin \left[k_{l}(z-c t)\right]-v_{z}\left\{B_{w} \cos \left(k_{w} z\right)+\frac{E_{l}}{c} \sin \left[k_{l}(z-c t)\right]\right\}\right)
$$




$$
\frac{d \gamma}{d t}=\frac{q}{m_{e} c^{2}} v_{x} E_{l} \sin \left[k_{l}(z-c t)\right]
$$

To find the energy gain in the FEL we must first solve for $v_{x}$. To do this we note that $E_{l}<<v_{z} B_{w}$ for FEL interactions. The transverse motion is dominated by the undulator field. We also take the approximation $v_{z} \simeq c$. We then have:

$$
v_{x}=-\frac{c K_{w}}{\gamma} \sin \left(k_{w} z\right)
$$

where $K_{w}=\frac{q B_{w} \lambda_{w}}{2 \pi m_{e} c}$ is the normalized undulator strength. We substitute this into equation 2.5 and similarly define the normalized laser field strength (also known as the normalized vector potential) $K_{r}=\frac{q E_{l} \lambda_{r}}{2 \pi m_{e} c^{2}}$. We then have:

$$
\frac{d \gamma}{d t}=-\frac{K_{r} K_{w} c k_{l}}{\gamma} \sin \left(k_{w} z\right) \sin \left[k_{l}(z-c t)\right]
$$

The electron motion satisfies $z(t)=v_{z} t+z_{0}$ and $v_{z}=c \sqrt{1-\frac{1}{\gamma^{2}}-\left(\frac{v_{x}}{c}\right)^{2}}$. Noting that $\gamma \gg 1$ and $v_{x} \ll c$ we can write:

$$
z-c t \cong z_{0}-\frac{z}{2}\left[\frac{1}{\gamma^{2}}+\left(\frac{v_{x}}{c}\right)^{2}\right]
$$

Equation 2.7 becomes:

$$
\frac{d \gamma}{d z}=\frac{K_{r} K_{w} k_{l}}{\gamma} \sin \left(k_{w} z\right) \sin \left(\frac{k_{l} z}{2}\left[\frac{1}{\gamma^{2}}+\left(\frac{v_{x}}{c}\right)^{2}\right]\right) \cos \left(k_{l} z_{0}\right)
$$

Here, we have taken $d / d t \approx c d / d z$. Notice in equation 2.9 we have a product of two sinusoidal oscillations. Integrated over many oscillations, this will average to zero unless:

$$
k_{w}=\frac{k_{l}}{2}\left[\frac{1}{\gamma^{2}}+\left(\frac{v_{x}}{c}\right)^{2}\right]
$$

We thus have a resonance condition for FELs and IFELs between the particle and laser field. Physically, this resonance occurs when the particle slips with respect to the laser field one optical cycle for every oscillation in the undulator field. When this occurs, for a particle in phase $\left(z_{0}=2 \pi n / k_{l}\right)$, the particle's transverse motion is 
always in the same direction as the laser electric field and thus significant acceleration occurs.

To find the energy gain over one period of the undulator we take the average value of $v_{x}^{2}$. From equation 2.6 we see that $\overline{v_{x}^{2}}=\left(c K_{w} / \gamma\right)^{2} / 2$ where the factor of 2 is to account for averaging the sine squared over one period ${ }^{1}$. The resonance equation becomes:

$$
k_{w}=\frac{k_{l}}{2 \gamma^{2}}\left(1+\frac{K_{w}^{2}}{2}\right)
$$

Substituting this resonant condition into 2.9 and integrating over one period gives us the energy gain.

$$
\Delta \gamma=\frac{2 \pi K_{r} K_{w}}{\gamma} \frac{k_{l}}{k_{w}} \cos \left(k_{l} z_{0}\right)
$$

Alternatively an effective gradient can be defined as:

$$
G_{e f f}=0.511 M V / m * \frac{\Delta \gamma}{\lambda_{w}}=0.511 \frac{k_{l} K_{r} K_{w}}{\gamma}[M V / m]
$$

Equations 2.11 and 2.12 combine to set the undulator strength, period, and number of periods for a given beam energy, desired modulation strength, and laser power available.

\section{$2.2 \quad$ IFEL Interaction Bandwidth}

In the prior sections we assumed a simple sinusoidally varying undulator field. In reality the magnetic field is more complex, having variations due to its finite length, differing periodicity in the end sections, and variations across the gap. In addition, the electron beam itself may have departures from the resonance condition, either due to the finite energy spread of the beam, shifting of the energy centroid, or position jitter that will sample different parts of the undulator field shot-to-shot. It therefore

\footnotetext{
ics.

${ }^{1}$ We will show in section 2.3 that removing this approximation yields coupling to higher harmon-
} 
becomes important to consider the tolerances of the IFEL interaction due to field variations and off-energy particles.

First, let us consider an undulator having an $N$ period sinusoidal field with a hard cut-off. To obtain equation 2.12 we assumed the particle is on-resonance. If we drop this assumption we have the energy gain defined by the integral:

$$
\Delta \gamma=\frac{q E_{0}}{m c^{2}} \frac{K_{w}}{\gamma} \int_{0}^{N \lambda_{w}} \cos \left[\frac{k_{l} z}{2 \gamma^{2}}\left(1+\frac{K_{w}^{2}}{2}\right)\right] \cos \left(k_{w} z\right) d z
$$

The product of the two cosines is decomposed into a rapidly varying part that integrates to zero and a slow oscillating term:

$$
\begin{array}{r}
\Delta \gamma=\frac{K_{r} K_{w}}{\gamma} k_{l} \int_{0}^{N \lambda_{w}} \cos \left[\frac{k_{l} z}{2 \gamma^{2}}\left(1+\frac{K_{w}^{2}}{2}\right)-k_{w} z\right] d z \\
\Delta \gamma=\frac{2 \pi N K_{r} K_{w}}{\gamma} \frac{k_{l}}{k_{w}} \operatorname{sinc}\left\{2 \pi N\left[\frac{k_{l}}{2 k_{w} \gamma^{2}}\left(1+\frac{K_{w}^{2}}{2}\right)-1\right]\right\}
\end{array}
$$

We see then that we have a sinc function about the resonance with a width determined by the number of oscillations. For a short undulator used for energy modulation, the energy acceptance is quite large. Using this simple argument, we can estimate that the three period E163 undulator resonant at $60 \mathrm{MeV}$ will modulate electrons within $3 \mathrm{MeV}$ of resonance. In actual fact, the resonance is further broadened by the undulator's departures from a simple sinusoid at the ends of the undulator.

In addition to the finite length, the undulator field varies in strength across the gap. It can be shown[34] that this variation goes as:

$$
B_{y}(y, z)=B_{0} \sin \left(k_{w} z\right) \cosh \left(k_{w} y\right)
$$

We can now estimate our tolerance to mis-steering away from the center of the gap. By substituting $K_{w}=K_{w 0} \cosh \left(k_{w} y\right)$ into 2.16 we see that for our three period undulator, the interaction will fall off by $\sim 10 \%$ when the beam is off center by 
$1.2 \mathrm{~mm}$. This is also much greater than the nominal beam transverse size, typically $<200 \mu \mathrm{m}$. Therefore, all electrons will experience roughly the same undulator field.

\subsection{Higher Harmonic Interactions}

To get the resonance equation 2.11 we period-averaged the transverse motion. This was then substituted into eqn. 2.9 to find the energy modulation at resonance, eqn. 2.12. If instead we keep the $\mathrm{z}$ dependent transverse velocity we can find a more precise expression for the laser interaction. Recall that the horizontal and longitudinal particle velocities are given by:

$$
\begin{aligned}
& v_{x}=-\frac{c K_{w}}{\gamma} \sin \left(k_{w} z\right) \text { and } \\
& v_{z}=c \sqrt{1-\frac{1}{\gamma^{2}}-\left(\frac{v_{x}}{c}\right)^{2}} .
\end{aligned}
$$

We combine these together, noting that $\gamma \gg 1$ and $v_{x} \ll c$, to obtain:

$$
\begin{aligned}
& v_{z}=c \sqrt{1-\frac{1}{\gamma^{2}}-\left(\frac{K_{w}}{\gamma}\right)^{2} \sin ^{2}\left(k_{w} z\right)} \\
& v_{z}=c \sqrt{1-\frac{1}{\gamma^{2}}\left(1+\frac{K_{w}^{2}}{2}\right)+\frac{K_{w}^{2}}{2 \gamma^{2}} \cos \left(2 k_{l} z\right)} \\
& v_{z} \approx c\left[1-\frac{1}{2 \gamma^{2}}\left(1+\frac{K_{w}^{2}}{2}\right)\right]+\frac{K_{w}^{2}}{4 \gamma^{2}} \cos \left(2 k_{l} z\right)
\end{aligned}
$$

Here, we have used the double angle formula. The next integration to get the particle position produces elliptic integrals in $z(t)$ [35]. However, since $K / \gamma \ll 1$ we can simplify this to:

$$
z-c t \approx z_{0}-c t\left[\frac{1}{2 \gamma^{2}}\left(1+\frac{K_{w}^{2}}{2}\right)\right]+\frac{K_{w}^{2}}{4 k_{w} \gamma^{2}} \cos \left(2 k_{w} c t\right)
$$


We can then substitute this and Eqn. 2.6 into equation 2.5 to obtain:

$$
\frac{d \gamma}{d t}=-\frac{K_{w} K_{r} k_{l} c}{\gamma} \sin \left(k_{w} c t\right) \operatorname{Im}\left\{\exp \left[i \frac{k_{l} c t}{2 \gamma^{2}}\left(1+\frac{K_{w}^{2}}{2}\right)-i \frac{K_{w}^{2}}{4 \gamma^{2}} \frac{k_{l}}{k_{w}} \cos \left(2 k_{w} c t\right)+i \phi\right]\right\}
$$

In Eqn. 2.6 we have taken the approximation $z(t) \approx$ ct. Equation 2.24 can be solved with a Bessel function expansion[36] to obtain the period averaged energy gain:

$$
\Delta \gamma=-\frac{2 \pi K_{w} K_{r}}{\gamma} \frac{k_{l}}{k_{w}}\left[J_{n+1}\left((2 n+1) \xi_{0}\right)+J_{n}\left((2 n+1) \xi_{0}\right)\right] \cos (\phi+n \pi),
$$

where $n$ satisfies:

$$
2 n+1=\frac{k_{l}}{2 \gamma^{2} k_{w}}\left(1+\frac{K_{w}^{2}}{2}\right)
$$

and,

$$
\xi_{0}=\frac{K_{w}^{2}}{2\left(1+K_{w}^{2} / 2\right)}
$$

Thus there are multiple arrangements of $k_{l}, k_{w}, \gamma$, and $K_{w}$ that give significant energy modulation, however only in odd multiples of the fundamental resonance condition. This is well known from free electron lasers where radiation is observed at the odd harmonics of the fundamental lasing wavenumber $k_{l}$. This effect can be thought of physically as coming from the symmetric figure-eight motion of the electrons in the undulator field.

FELs (and conversely IFELs) can also couple to even harmonics when the symmetry of the figure-eight motion is broken. This is done simply by adding a net transverse motion to the electrons through the undulator. The theory for the even coupling was first derived by Colson, Dattoli, and Ciocci[35] for FELs and later applied to IFELs by P. Musumeci[37]. The derivation follows similar steps used for the symmetric, odd harmonics only case. It will not be re-derived here, but the equation for the energy gain per period is given as it will be used in later sections. 


$$
\Delta \gamma=\frac{2 \pi K_{r} K_{w}}{\gamma} \frac{k_{l}}{k_{w}} \cos \phi \sum_{m=-\infty}^{\infty} J_{m}(n \xi)\left[J_{2 m+n+1}(n Z)+J_{2 m+n-1}(n Z)\right]
$$

where $\xi=K_{w}^{2} / 2\left(1+K_{w}^{2} / 2+\gamma^{2} \theta^{2}\right)$ and $Z=2 K_{w} \gamma \theta /\left(1+K_{w}^{2} / 2+\gamma^{2} \theta^{2}\right)$. Here, $\theta$ is the additional angle of the electrons. Note for $\theta=0$ we regain equation 2.25. The resonance equation now includes all harmonics:

$$
k_{w}=\frac{k_{l}}{2 n \gamma^{2}}\left(1+\frac{K_{w}^{2}}{2}\right)
$$

\subsection{Microbunching in a Chicane}

After the IFEL the electrons pass through a dispersive chicane to transform the energy modulation into a longitudinal density modulation. The equations for bunching of electron beams were first formulated for non-relativistic beams in klystrons[38]. In klystrons, bunching is from velocity modulation and time-of-flight. Here, the chicane replaces the velocity modulation and instead varies the path-length of electrons based on their modulated energy. Since both processes are linear, however, the same equations apply. The longitudinal density modulation $\rho$ can be written as a cosine expansion with Bessel function coefficients.

$$
\rho(z)=\rho_{0}\left[1+2 \sum_{n=1}^{\infty} J_{n}(n \beta) \cos \left(n k_{l} z\right)\right]
$$

Here $\beta=k_{l} R_{56} \eta / \gamma_{0}$ describes the degree of bunching where $\eta$ is the normalized amplitude of IFEL modulation, $\gamma_{0}$ is the normalized average beam energy, and $\rho_{0}$ is the initial density of the beam. $R_{56}$ is the dispersion of the chicane, which can be defined through magnetic measurements and is described in greater detail in §3.1. Equation 2.30 can be further refined[39, 40] to include an initial energy spread, $\sigma_{\gamma}$. 


$$
\begin{aligned}
\rho(z) & =\rho_{0}\left[1+2 \sum_{n=1}^{\infty} b_{n} \cos \left(n k_{l} z\right)\right] \\
b_{n} & =J_{n}\left(n k_{l} R_{56} \frac{\eta /}{\gamma_{0}}\right) \exp \left[-\frac{1}{2}\left(\frac{n k_{l} R_{56} \sigma_{\gamma}}{\gamma_{0}}\right)^{2}\right]
\end{aligned}
$$

Here, we have separated out the Fourier series coefficients, $b_{n}$. These are termed the 'bunching coefficients', and are often quoted as a figure-of-merit for bunching in FELs and IFELs. Optimal bunching occurs when the bunching coefficients are maximized, which in turn occurs for $\beta=1-1.5$ depending on the harmonic number. For $\beta=1$, we can write the expression in the exponent as: $n k_{l} R_{56} / \gamma_{0}=n / \eta$. We then see that when the IFEL modulation $\eta$ is less than initial energy spread $\sigma_{\gamma}$ the microbunching begins to wash out. Further attenuation terms can be included to account for electron beam divergence and laser wavefront curvature. These, however, tend to be much smaller corrections compared to the initial energy spread term. Figure 2.2 shows a plot of the longitudinal distribution for maximum bunching and $\eta / \sigma_{\gamma}=2.5$.

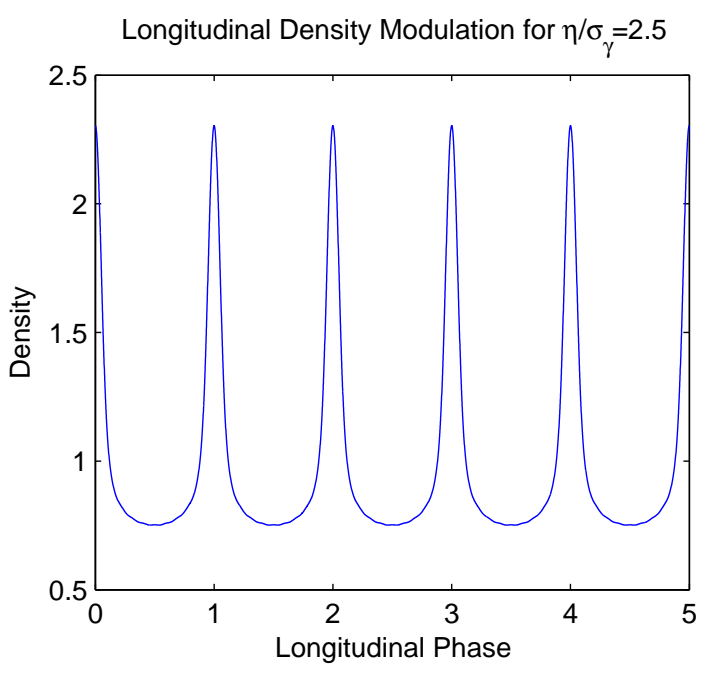

Figure 2.2: Plot of 2.31 with $\eta / \sigma_{\gamma}=2.5$ and $\beta=1$. Density is relative to the initial density $\left(\rho_{0}=1\right)$. 


\subsubsection{Microbunch Washout}

To make use of the microbunches for acceleration they must survive a drift section between the chicane and accelerator sections. There is the possibility that in this drift the microbunches may washout.

There are two effects that can contribute to microbunch washout. The first is space charge forces that drive a density oscillation at the plasma frequency [41]. Longitudinal space charge becomes a concern when the drift distance $L$ approaches a quarter of the plasma oscillation period $c / \omega_{p}$. Equation 2.33 gives the relativistically corrected plasma frequency $\omega_{p}$ where $e, m_{e}$, and $n$ are the electron charge, mass, and density and $\epsilon_{0}$ is the free-space permittivity.

$$
\omega_{p}=\sqrt{\frac{e^{2} n}{m_{e} \epsilon_{0} \gamma^{3}}}
$$

For a $50 \mathrm{pC}, 1 \mathrm{ps}$ long electron pulse with a spot size of $\sim 100 \mu \mathrm{m}$ we obtain a plasma wavelength of $67 \mathrm{~cm}$. This compares to a drift distance used in the experiment of $\leq 10 \mathrm{~cm}$. In the actual experiments charge was actually decreased due to energy collimation 5.2 to $\sim 5 \mathrm{pC}$ making space charge in the experiment even less of an issue.

The second cause of microbunch washout is a second order phase slippage due to beam divergence $\left(T_{522}\right.$ and $\left.T_{544}\right)$. The electron pulse is focused through the microbunching hardware before reaching the imaging point of a spectrometer. A particle that has some divergence angle relative to the nominal beam trajectory, either due to the beam emittance or an explicit focusing, will slip relative to the nominal beam trajectory (figure 2.3).

The effect of this slippage can quantified by deriving an attenuation term analogous to washout term due to the initial energy spread (equation 2.31). We consider the beam as a set of $i$ beamlets, each propagating at an angle $\theta_{i}$. Each beamlet propagates a distance $L$ from the undulator, through the chicane forming microbunches with the longitudinal density given by:

$$
\rho_{i}=\rho_{0, i}\left\{1+2 \sum_{n=1}^{\infty} J_{n}(n \beta) \cos \left[n k_{l}\left(z-1 / 2 L \theta_{i}^{2}\right)\right]\right\}
$$




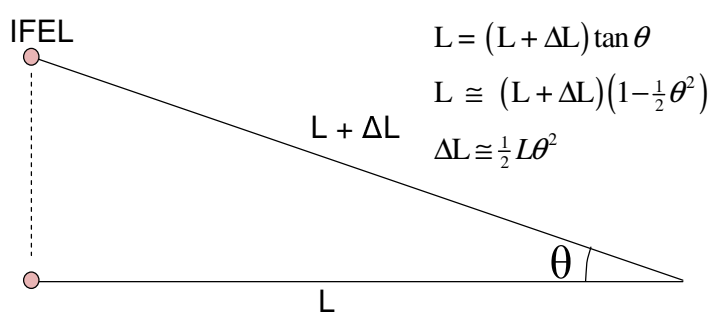

Figure 2.3: Microbunch washout due to divergence. A particle traveling at some angle $\theta$ relative to the nominal beam direction will slip behind relative to the nominal trajectory.

Here, $z$ is the longitudinal position within the bunch. Each beamlet is delayed relative to the nominal beam center due to its angle $\theta_{i}$. To find the total longitudinal charge density we convert the finite sum into an integral. The charge of each beamlet $\rho_{i}$ is defined by the rms spread of divergence angles within the beam, $\sigma_{\theta}$. Thus, $\rho_{i} \Rightarrow \rho_{0} d \theta /\left(\sqrt{2 \pi} \sigma_{\theta}\right) \exp \left[-\theta^{2} / 2 \sigma_{\theta}^{2}\right]$. We can now write the total longitudinal charge density.

$$
\begin{aligned}
\rho & =\rho_{0}\left\{1+2 \sum_{n=1}^{\infty} J_{n}(n \beta) \cos \left(n k_{l} z\right) \int_{-\infty}^{\infty} \frac{d \theta}{\sqrt{2 \pi} \sigma_{\theta}} e^{-\frac{\theta^{2}}{2 \sigma_{\theta}^{2}}} \cos \left(n k_{l} \frac{L \theta^{2}}{2}\right)\right\} \\
\rho & =\rho_{0}\left\{1+2 \sum_{n=1}^{\infty} J_{n}(n \beta) \cos \left(n k_{l} z\right) \sqrt{\frac{1+b_{n}}{2 b_{n}^{2}}}\right\} \\
b_{n} & =\sqrt{1+n k_{l} L \sigma_{\theta}^{2}}
\end{aligned}
$$

From equation 2.34 the cos term is split via the difference rule with the resulting sin term integrating to zero yielding equation 2.35. We therefore see that much like the initial energy spread, beam divergence causes a washing-out of the microbunch 
structure and more strongly effects higher harmonics of the longitudinal bunch density.

In the experiment, the beam is focused ahead of the undulator and reaches a focus of $\sim 100 \mu \mathrm{m}$ some $30 \mathrm{~cm}$ after the undulator. The rms angle spread to attain this focus is $\sim 2 \times 10^{-4}$ radians assuming an emittance of $2 \mathrm{~mm}$-mrad. Defining the divergence washout attenuation term as:

$$
\delta_{\theta, n}=\sqrt{\frac{1+b_{n}}{2 b_{n}^{2}}} .
$$

we have $b_{1}=1.046$ and $\delta_{\theta, 1}=0.97$ at the first harmonic. Thus, the washout due to beam divergence is negligible for this focusing. As we shall see in chapter 8 , when stronger focusing is introduced, the washout due to divergence becomes important.

\subsection{Simulation of Microbunching Experiment}

The analytic results of $\S 2.1$ and $\S 2.3$ are useful in setting the initial design parameters and calculating approximately the expected results of experiments. However, for true comparison to experiment, it is necessary to include the true Gaussian laser profile and fringe fields of the undulator. For these, an analytic result is unachievable, and we instead turn to particle tracking simulations for finding predictions of experiment. A simple particle tracking code integrating the Lorentz equations was written for the purpose. The code uses the analytic form for a $T E M_{00}$ mode laser, and a field map of the undulator exported from the magnetic codes (these will be described in further detail in the next chapter). It does not include absorption of laser by the electrons (negligible in the case of a short undulator for microbunching) or space charge effects. Separate simulations were performed using Genesis[42] which selfconsistently tracks the optical field to verify that the effect on the optical field is negligible. Other simulations using Elegant's[43] LSCDRIFT were done to find the effect of space charge on the resulting microbunches. These simulations showed that microbunches begin to degrade after around $0.5 \mathrm{~m}$, consistent with the discussion of §2.4.1. The primary difference found in using a full $3 \mathrm{~d}$ simulation over the analytic 
equations comes from the roll-off in laser intensity transversely. To obtain sufficiently high peak fields, the laser spot size is roughly the same size as the electron beam, meaning that electrons near the edge of the beam will obtain less modulation in the IFEL and therefore less bunching in the chicane. Actual results of simulation will be shown along side the experimental data for comparison. 


\section{Chapter 3}

\section{Hardware Design and Fabrication}

\subsection{Field Integrals of Magnets}

In addition to the constraints imposed on the magnet design by the physics of the laser electron interaction given in chapter 2, the microbunching hardware should follow other sensible requirements that keep the beam trajectory well confined. Ideally, the outgoing trajectory should match the incoming with no net kick horizontally or vertically. In addition, offsets, overall shifts in horizontal or vertical position, should be minimized. This is particularly important in the undulator where the electrons must remain overlapped transversely with the laser to maintain the interaction. These two values, net kick and transverse offset, are quantified by the integrals of the magnetic field of the microbunching hardware and follow directly from consideration of the Lorentz force equation. The first integral (3.2) is proportional to the transverse angle of the electron beam. Integrating a second time gives the second integral (3.4) proportional to the offset. The magnets are designed in such a way as to zero these two field integrals after the exit of the magnets, as $z \rightarrow \infty$. 


$$
\begin{aligned}
x^{\prime}(z) & =\frac{e}{\gamma m c} 1^{s t}(z) \\
1^{s t}(z) & =\int_{-\infty}^{z} B_{y}\left(z^{\prime}\right) d z^{\prime} \\
x(z) & =\int_{-\infty}^{z} x^{\prime}\left(z^{\prime}\right) d z^{\prime} \\
2^{n d}(z) & =\int_{-\infty}^{z} d z^{\prime} \int_{-\infty}^{z^{\prime}} B_{y}\left(z^{\prime \prime}\right) d z^{\prime \prime}
\end{aligned}
$$

For the chicane there is an additional field integral that can be defined that helps quantify the creation of microbunches from the energy modulation imparted by the IFEL. In particular, we want to know the longitudinal (z) position shift of an electron within the bunch based on its energy. This quantity is known as the $R_{56}$ where $R$ is the transfer matrix giving the mapping to first order of the six dimensional phase space before and after a beamline element. The distance traversed $L$ over a longitudinal distance $z$ is given by

$$
\begin{aligned}
L & =\int_{0}^{z}\left[1+\left(\frac{d x\left(z^{\prime}\right)}{d z^{\prime}}\right)^{2}\right]^{\frac{1}{2}} d z^{\prime} \\
& =\int_{0}^{z}\left[1+\left(\frac{e}{\gamma m c} 1^{s t}\left(z^{\prime}\right)\right)^{2}\right]^{\frac{1}{2}} d z^{\prime}
\end{aligned}
$$

If we now take the difference of an off energy particle compared to a nominal trajectory we find for small angular deflections $\left(x^{\prime} \ll 1\right)$ : 


$$
\begin{aligned}
L_{1}-L_{0} & =\int_{0}^{z}\left[1+\left(\frac{e}{\gamma_{1} m c} 1^{s t}\left(z^{\prime}\right)\right)^{2}\right]^{\frac{1}{2}}-\left[1+\left(\frac{e}{\gamma_{0} m c} 1^{s t}\left(z^{\prime}\right)\right)^{2}\right]^{\frac{1}{2}} d z^{\prime} \\
& \approx \int_{0}^{z} 1+\frac{1}{2}\left(\frac{e}{\gamma_{1} m c} 1^{s t}\left(z^{\prime}\right)\right)^{2}-1-\frac{1}{2}\left(\frac{e}{\gamma_{0} m c} 1^{s t}\left(z^{\prime}\right)\right)^{2} d z^{\prime} \\
\Delta L & =-\left(\frac{e}{\gamma_{0} m c}\right)^{2}\left(\frac{\Delta \gamma}{\gamma_{0}}\right) \int_{0}^{z}\left[1^{s t}\left(z^{\prime}\right)\right]^{2} d z^{\prime}
\end{aligned}
$$

In the last step we have taken $\gamma_{1} \equiv \gamma_{0}+\Delta \gamma$. Notice that the slippage $\Delta L$ is negative for particles with energy higher than the beam. That is, those particles travel a shorter total distance through the magnet compared to the mean particle and will therefore arrive ahead of the nominal trajectory. The particle position within the bunch will then change by $\Delta z=-\Delta L$.

In addition to the differing particle paths through the chicane, particles at different energies also travel at slightly different velocities. This velocity difference leads to an additional time-of-flight induced path length difference, $\Delta z_{T O F}$ given by:

$$
\Delta z_{T O F}=\left(v_{1}-v_{0}\right) t_{0}=\left(v_{1}-v_{0}\right) \frac{L}{v_{0}}
$$

A relativistic particle's velocity is related to $\gamma$ by $v=c \sqrt{1-1 / \gamma^{2}} \approx c\left(1-\frac{1}{2 \gamma^{2}}\right)$. We then have:

$$
\Delta z_{T O F}=L \frac{\Delta \gamma}{\gamma^{3}}
$$

where $L$ is the total distance traveled. The $R_{56}$ is then defined as $\Delta z /(\Delta \gamma / \gamma)$, giving us eq 3.12 .

$$
R_{56}=\frac{L}{\gamma^{2}}+\left(\frac{e}{\gamma m c}\right)^{2} \int_{-\infty}^{\infty}\left[1^{s t}(z)\right]^{2} d z
$$

We therefore have three expressions that further constrain the designs of the undulator and chicane based on the electron trajectories. With these and the design 
criteria set by the physics of the IFEL and bunch formation processes we can begin the design the hardware.

\subsection{Design Considerations for LEAP/E-163}

The design process for the microbunching hardware began by considering the available beam parameters from the NLCTA and driving laser for the experiment. In particular, the $60 \mathrm{MeV}$ beam energy combined with the 800nm wavelength of the Ti:Sapphire sets the undulator period at around $2 \mathrm{~cm}$ from the FEL resonance equation (eq 2.29). Furthermore, current permanent magnet materials have $B_{r} \simeq 1.25 \mathrm{~T}$ limiting the undulator parameter $K_{w}$ to $\approx 0.5-1.2$ depending on the gap of the undulator. A second criteria set for the microbunching hardware is that the IFEL interaction should not be so strong as to obscure any interaction from the downstream accelerating cells. For the initial, small interaction experiments like the ITR setup[26] this limit is around 60-100 keV. From the FEL gain equation (2.5) this sets the number of periods to just three given a reasonable laser energy of $0.5 \mathrm{~mJ} /$ pulse in a 1 ps pulse.

The field integrals through the undulator set additional constraints on the design. As mentioned in the previous section, optimally both field integrals should be zero to maintain overlap between the laser and electrons and to keep the electron trajectory the same before and after the undulator. The first integral can be zeroed easily by keeping the undulator antisymmetric in z. Of course, non-uniformities in the magnet block strengths or other manufacturing flaws can lead to small kicks. This can be accounted for by adding a tuning mechanism into the design. This will be discussed in more detail in $\S 3.5$.

The second integral is much harder to deal with, and must be tuned to zero iteratively during the simulation phase by adjusting the thickness of the first few and final few blocks of the undulator. For a hybrid-Halbach undulator[44] used in this design there are three generally prescribed means of getting a zeroed second integral. In the first option, the first and last half period are reduced in thickness to about $\frac{1}{2}$ the original thickness giving effectively a half kick at the first and last poles. A somewhat more refined design varies both the first and second half periods to $\frac{1}{4}$ and 
$\frac{3}{4}$ thickness. Figure 3.1 shows a comparison of these two options.

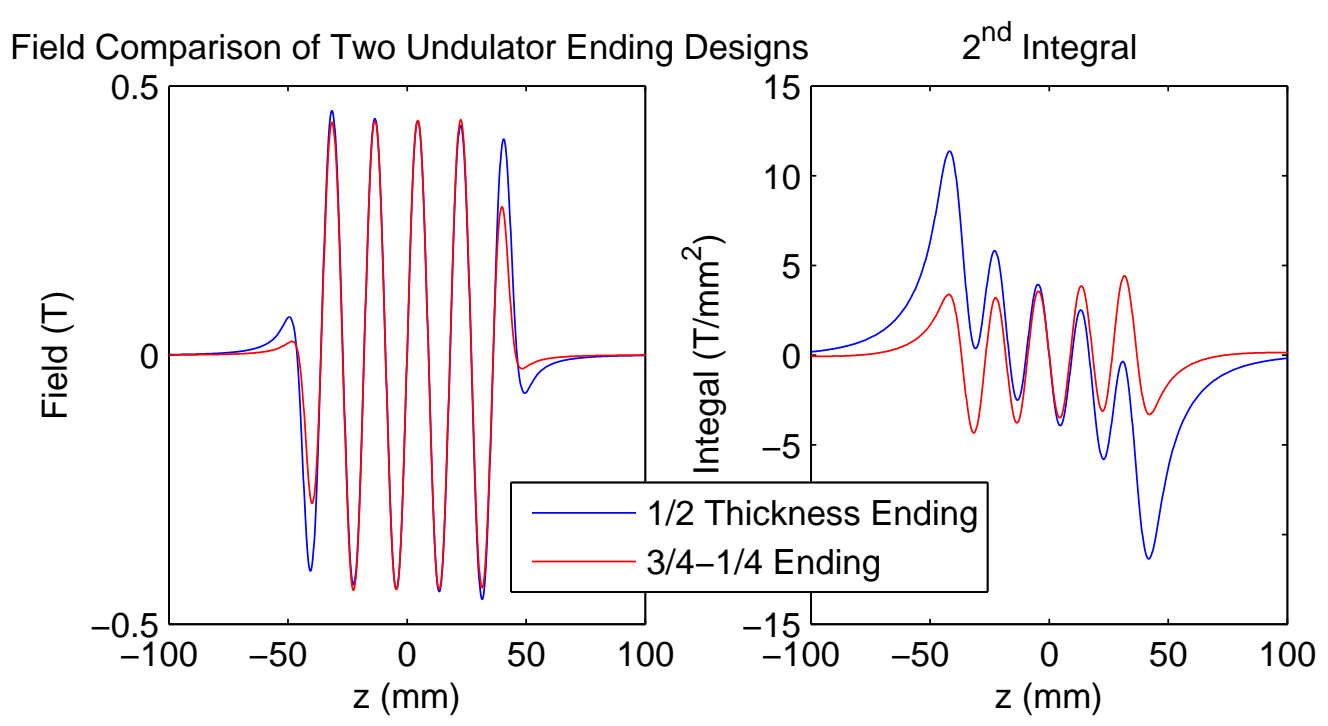

Figure 3.1: The $2^{\text {nd }}$ Field Integral.

The $\frac{1}{4}-\frac{3}{4}$ ending does a better job of minimizing the electron displacement and maintaining overlap with the laser field. However this is at the expense of additional fabrication complexity that requires slicing a very thin block of magnetic material. An additional problem with both these options is that neither can be tuned to adjust for strength errors. A way to add tuning is to make the final magnet block larger and then remove field flux using an iron shorting plate. The undulator is then tuned by adjusting the distance of the shorting plate from the final pole face with shims.

After the undulator is specified, a few important design parameters for the chicane can also be determined. The key number in describing the chicane is the dispersion $R_{56}$, the correlation between the initial energy and final temporal offset of a particle in the bunch. The IFEL will impart a modest energy modulation of about $60 \mathrm{keV}$ or $0.1 \%$ peak-to-peak. For this modulation we want a shift in longitudinal position of one half the optical wavelength $(800 \mathrm{~nm})$ in order to microbunch. Therefore the $R_{56}$ should be $\sim 0.4 \mathrm{~mm}$. Using equation 3.12 we can evaluate chicane designs to get the needed dispersion.

A few further design criteria come up when considering the nature of the experiment arrangement. The experiment requires lenses near the electron beam for 
introducing the laser, diagnostics for overlapping the two beams, and compactness in the longitudinal direction to keep spot sizes small and limit wash-out of the microbunches. These all push for placing everything in a single, large vacuum chamber rather than having the magnets in air with small vacuum chambers in their gaps as is usually done. The undulator and chicane must therefore be vacuum compatible. Furthermore, to cover a range of possible beam energies, both electron and laser, both components are designed to be tunable. The undulator is tuned by adjusting the gap while the chicane is adjusted by including electromagnetic coils as well as permanent magnets.

\subsection{Simulation Software: Radia and Mafia}

Design efforts began using the magnetostatic solver included in the package Mafia[45]. The package includes a GUI for defining the geometry and materials. Since the problem is static, it can be reduced to solving for a potential at each point on the grid. The fields are then expressed as a gradient of the potential. Mafia also includes a PIC code for tracking particles through the static field. This was used initially for emittance studies. However, since the PIC calculation includes many unnecessary steps - calculating wakes, scattering of the particle field, and space charge for example - it proved far quicker to export a field map of the magnetostatic fields to a second code that did solely particle tracking without including those other steps.

Simulation tolerance studies to check for effects on the on-axis undulating field included random variations of the polarization angle of each magnet block, small variations in iron block gaps and thickness, and magnet strengths. It was found that the on-axis field was relatively tolerant to magnet block variations. This is due in large part to the fact that the undulator is 'iron dominant', meaning that the field shape is dictated mainly by the iron pieces. The primary error by the variations was a non-zero first integral which could corrected by adjusting the end plates.

Each run of Mafia took 1-2 hours depending on the resolution settings. This made an iterative process like tuning the end magnet thicknesses a time consuming process. Instead of dealing with this time penalty, design efforts switched to using 
the code Radia[46], an add-on package that uses Mathematica as an interface. Radia is solely a magnetostatic solver intended for designing beamline insertion devices like the undulator. The code uses the Boundary Element Method ${ }^{1}$. BEM is applicable to problems where a set of partial differential equations can be recast as a mathematically equivalent set of integral equations on a boundary, as is the case in magnetostatics. Radia therefore only needs to discretize the surfaces rather than an entire 3D grid. This yields a significant speed up compared to Mafia. The advantage is even more striking when calculating fields on the outside of a magnetic device where the volume is effectively infinite.

Radia was used to complete the undulator design by finding the correct thicknesses of the end magnets and the spacing of the end plate needed to zero the second integral. Radia was also used to design the chicane. The CAD images shown later in figures 3.2 and 3.3 are from Radia. The surface discretization can be made out as thin black lines in the images. Typically, a greater discretization is needed for the iron blocks since the field strength and orientation can change rapidly near the surface of the iron.

\subsubsection{Particle Tracking through Fields}

To study the effects of the magnets on the beam beyond the simple analytic treatment described in chapter 2 , a particle tracking code was written to push particles through magnetostatic fields exported from the magnet simulation software Mafia \& Radia. Although Mafia and Radia both have their own particle tracking routines built-in, these were found to be too slow to be of much use. The custom particle tracking code performed step-wise Euler integration of the Lorentz force equations. With this code, both the undulator and chicane were checked for aberrant focusing effects and emittance degradation to the beam. Results from these studies are presented in subsequent sections, but were found to largely negligible.

This code was later extended to include the laser field in order to simulate the IFEL interaction and subsequent bunching in the chicane.

\footnotetext{
${ }^{1}$ Also known as the Boundary Integral Method
} 


\subsection{Design Results}

\subsubsection{The Undulator}

The undulator is composed of blocks of Neodymium-Iron-Boride (NdFeB) with a residual field of $B_{r}=1.25 T^{2}$ and vanadium permendur as the 'iron' pole pieces ${ }^{3}$. Vanadium Permendur is an alloy of $\sim 50 / 50$ iron and cobalt with a small $(2 \%)$ amount of vanadium. This alloy has a higher magnetic flux saturation than low carbon steel. A greater flux concentration at the pole tips can be achieved by making the pole height lower than that of the magnets. It can be further increased by making the pole width smaller too, however, this was not done for this design in order to simplify the holder assembly. The end plates are made from low carbon steel and include an aperture for the beam. A CAD image from Radia is shown in figure 3.2. Table 3.1 gives the undulator geometry resulting from simulation.

\begin{tabular}{|l|c|}
\hline Parameter & Value \\
\hline Period & $1.8 \mathrm{~cm}$ \\
Number of Periods & 3 \\
Width & $40 \mathrm{~mm}$ \\
Magnet Height & $32 \mathrm{~mm}$ \\
Pole Height & $30 \mathrm{~mm}$ \\
Magnet Thickness & $5 \mathrm{~mm}$ \\
Pole Thickness & $4 \mathrm{~mm}$ \\
Gap Height & $4-15 \mathrm{~mm}$ \\
End Plate Offset & $4 \mathrm{~mm} *$ \\
End Plate Aperture & $8 \mathrm{~mm}$ \\
\hline
\end{tabular}

Table 3.1: List of undulator design specifications. (*Before correction. After correction the offset became $\sim 15 \mathrm{~mm}$.)

The discretization in Radia is fairly coarse throughout most of the geometry. The magnet blocks are subdivided $3 \times 3 \times 3$ sub-blocks and the poles $2 \times 3 \times 5$ in $x, y$, and $z$ (see figure 3.2). Greater discretization was studied and found unnecessary with one exception. Unfortunately, an initial check of the end plate aperture discretization

\footnotetext{
${ }^{2}$ Sumitomo $38 \mathrm{H}$

${ }^{3}$ Magnetic blocks and pole material provided by Roger Carr of SSRL
} 


\section{The E163 IFEL Undulator}
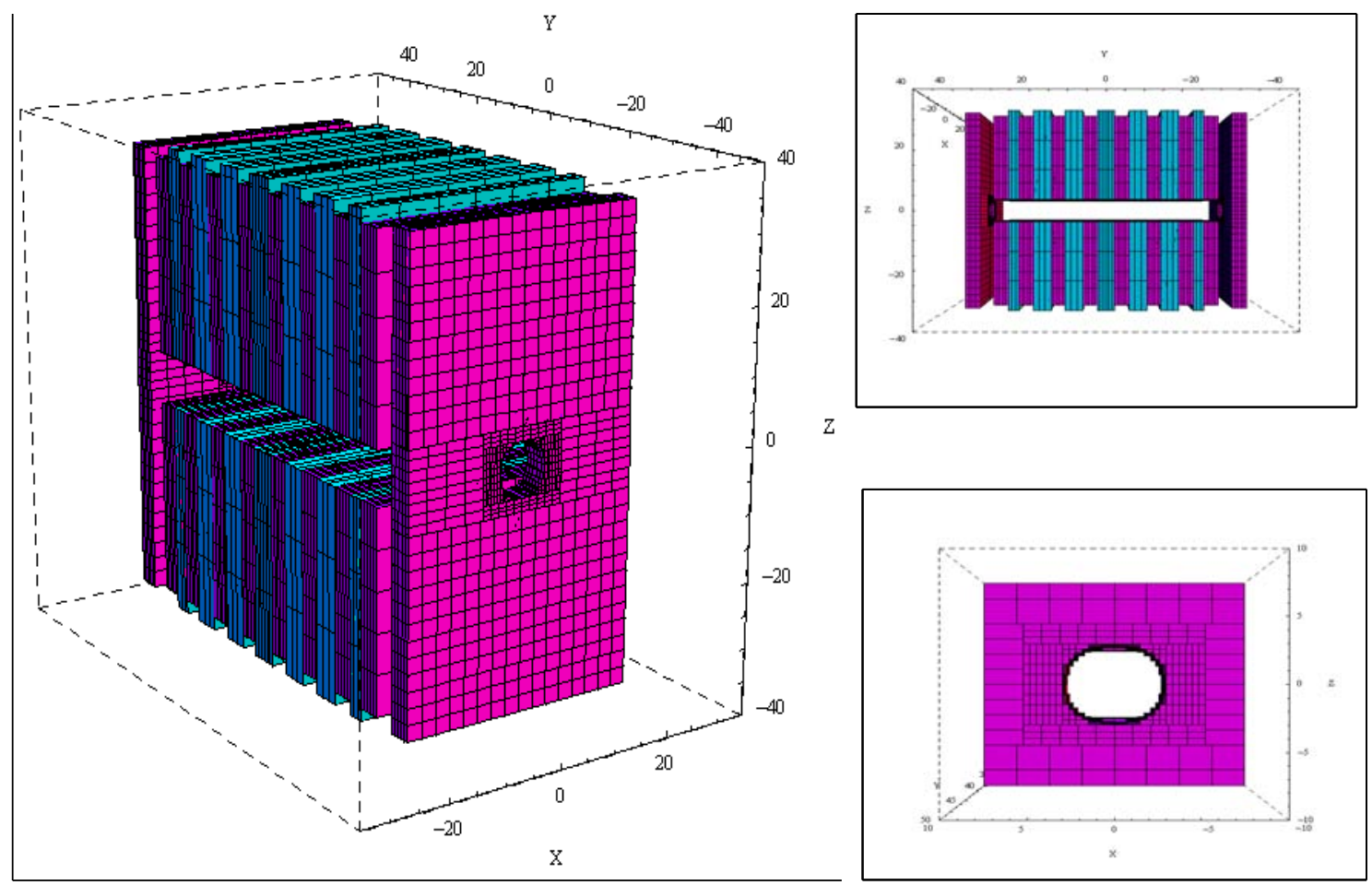

Figure 3.2: The undulator as initially designed in Radia. Blue is magnetic material, magenta the iron. The smaller insets (right) show the side view and aperture in the shorting plate. Black lines indicate discretization of the surfaces.

was neglected. This in fact led to a significant error in the end fields. Luckily, this was corrected by using a larger end plate offset. The undulator measurement and correction is described in more detail in $§ 3.5 .1$.

The end plate beam aperture was initially an $8 \mathrm{~mm}$ circular hole that was later increased to an $8 \mathrm{~mm} \times 20 \mathrm{~mm}$ rectangular aperture in order to afford greater clearance for the laser going to the ITR tape in the case of the net acceleration experiment. The laser for the ITR accelerator must pass through the undulator as well since the first turning mirror must be located sufficiently far away from the tape to avoid damage due to a small laser spot size near the focus. Simulation with Radia prior to modification showed no change to end plate spacing for the enlarged aperture. Essentially, 
since the flux through the end plate is so small, even with the enlarged aperture, the iron is able to contain and direct the flux around the aperture with little field leaking into the gap.

Undulators are naturally focusing in the vertical direction[34]. This is due to the fringing of the fields near the poles providing a $B_{z}$ component off-axis coupling to the horizontal motion of the electrons. For long undulators this sets up a betatron

oscillation in the vertical direction with a period $\lambda_{B}=\frac{\sqrt{2} \gamma}{K_{w} k_{w}}$. For typical values of $K_{w}=0.75, \gamma=100$, and $\lambda_{w}=2 \mathrm{~cm}$ this gives a betatron period of $4 \mathrm{~m}$. However, this undulator is only $10 \mathrm{~cm}$ in length, so instead the undulator acts as a weak thin lens. Integration of the fields from Radia give an effective focal length of $6.5 \mathrm{~m}$, negligible compared to strength of upstream focusing quadrupole triplet.

\subsubsection{The Chicane}

The chicane is made up of $3 \mathrm{H}$-magnets (named for the ' $\mathrm{H}$ ' shaped air space created by the iron pole shape). The inner H-magnet is roughly twice the thickness as the outer two to give a triangular offset trajectory to the electron beam. H-magnets were chosen over ' $\mathrm{D}$ ' shaped dipoles in order to gain greater field uniformity across the pole width and in turn limit emittance growth to the beam. The measured field uniformity is better than $0.02 \%$ over $\pm 10 \mathrm{~mm}$ transversely. The $\mathrm{H}$ magnets have permanent magnet in the back legs and four coils located in the top and bottom arms. With no current the field in the gap is $\sim 0.31 T$. The coils provide up to 300 Amp-turns. The coils are capable of providing a $\pm 15 \%$ variation in $R_{56}$ which can cover a $15 \%$ error in the IFEL energy modulation or $10 \%$ in the mean energy of the bunch (less due to the square dependence). The current adjustment was also used in the microbunching experiment (chapter 6) to maximize the bunching density. The permanent magnets are the same $\mathrm{NdFeB}$ material that is used in the undulator having a residual field $B_{r}=1.25 T$. The center-to-center separation between each dipole is $50 \mathrm{~mm}$ giving an effective length of the chicane of $12 \mathrm{~cm}$. Figure 3.4 shows the dipole field profile for a single H-magnet.

The gap for the chicane is fixed at $7 \mathrm{~mm}$, all poles have a width of $40 \mathrm{~mm}$ and 


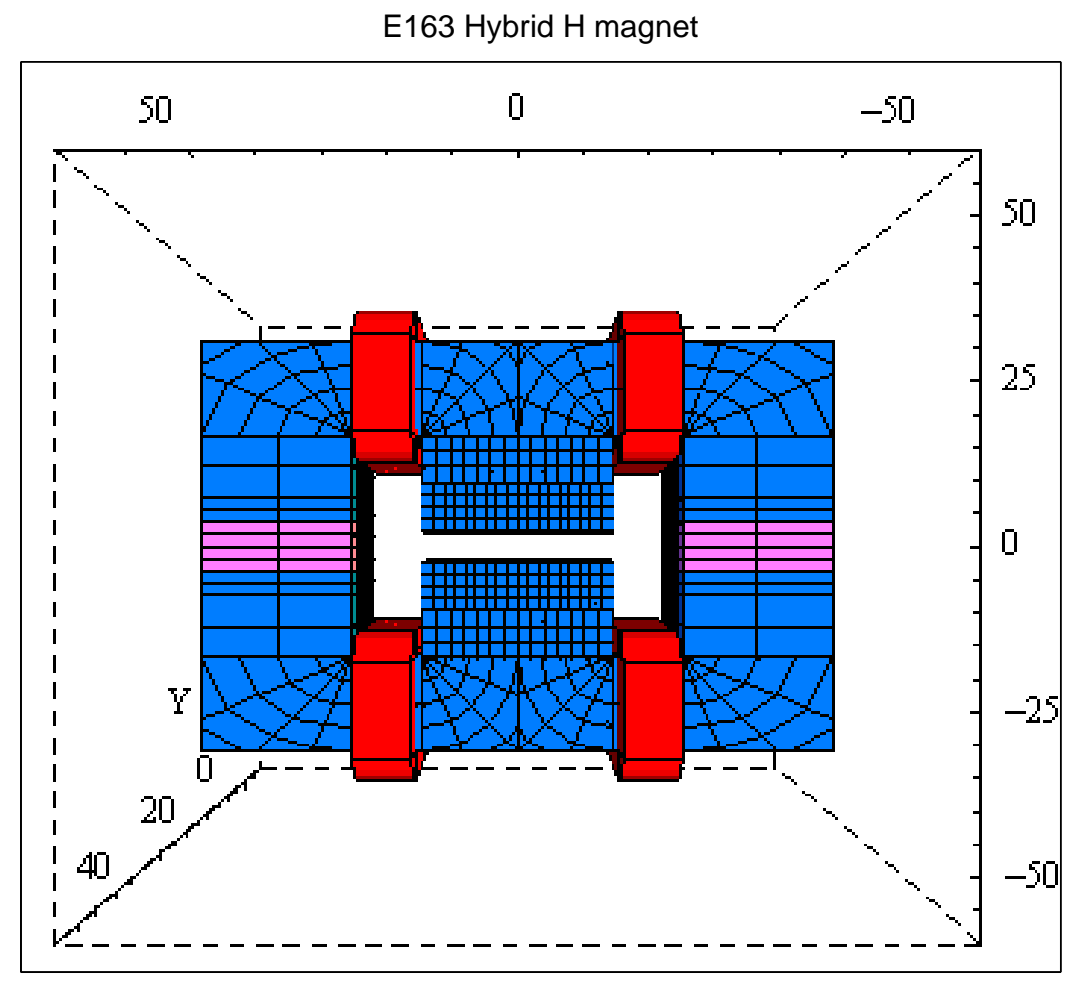

Figure 3.3: Radia CAD image of a single H-magnet. Blue is the iron, magenta the magnet, and red the coils. Black lines indicate discretization of surfaces in the simulation.

share a single axis even though the electrons will pass $2 \mathrm{~mm}$ off center through the center H-magnet. Particle tracking simulations show this will cause no problems. From the particle tracking we estimate for the normalized emittance growth through the chicane of $0.05 \mathrm{~mm}$-mrad in the vertical and $0.1 \mathrm{~mm}$-mrad in the horizontal, well below the estimated initial emittance $\epsilon_{N} \cong 4-m m-m r a d$. The focusing strength of the chicane is 3 meters in the vertical and -50 meters in the horizontal, also negligible compared to the focusing imposed by upstream quads.

\section{Chicane Thermal Issues}

Since the chicane will go into the vacuum rather than having a small aperture beam pipe, one of the chief concerns for the chicane becomes heat removal from the coils. 

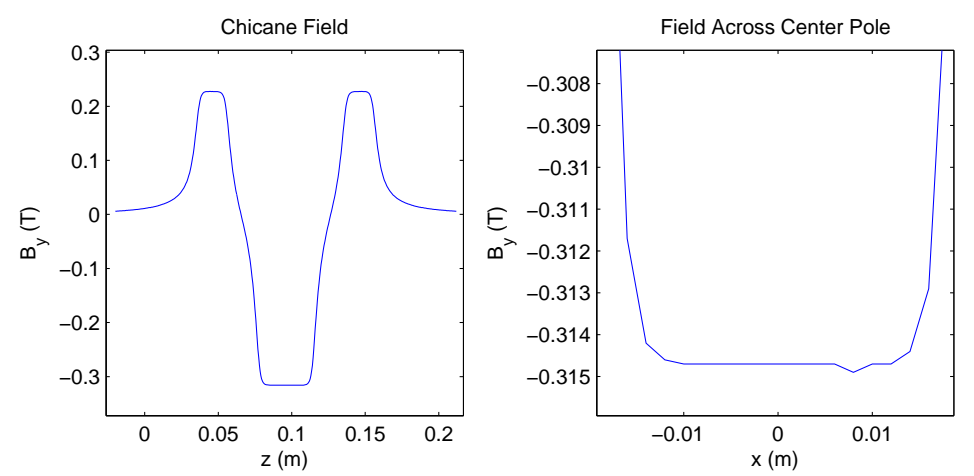

Figure 3.4: Measured field profiles of the chicane. Left: field on-axis along z. Right: field seen transversely parallel to the gap in the center H-magnet. For the region \pm 10 $\mathrm{mm}$ about the central axis the field variation is less than $0.0002 \mathrm{~T}$, the sensitivity limit of the Hall probe.

\begin{tabular}{|l|c|}
\hline Parameter & Value \\
\hline Gap & $7 \mathrm{~mm}$ \\
Outer H-mag Thickness & $19.5 \mathrm{~mm}$ \\
Inner H-mag Thickness & $38 \mathrm{~mm}$ \\
Pole Width & $40 \mathrm{~mm}$ \\
Back leg width & $32 \mathrm{~mm}$ \\
Coil turns & 160 \\
\hline
\end{tabular}

Table 3.2: List of chicane design specifications.

At a maximum current of 2 Amps to each coil, the heat generated will be about 22 Watts. Left with no heat removal system this would heat the chicane to around $110^{\circ} \mathrm{C}$ in vacuum, causing the magnets to demagnetize. To prevent this, a water cooling system is built into the magnet assembly. A single $\frac{1}{4}$ inch copper tube attached to the underside of the base plate circulates water. The base, side, and top plates are made of copper to aid in heat transfer from the coils to the water cooling tube. Tests in vacuum have found that while the coils themselves do still warm some $25^{\circ} \mathrm{C}$, the heating of the permanent magnet is just $2^{\circ} \mathrm{C}$ at 2 Amps of current. 


\subsection{CAD Design, Fabrication, and Testing}

After finding suitable magnetic layouts from simulation, designs were drawn up in SolidEdge[47] that could support the magnets while including the additional constraints laid out in $§ 3.2$. The undulator design includes shimmable end plates and an adjustable gap. The chicane design must incorporate thermal management to avoid demagnetization of the permanent magnets. At the same time the support structure parts have to have sufficiently tight tolerances to maintain alignment of the magnets. The fabrication tolerances were generally specified as $25 \mu \mathrm{m}$ and $2^{\circ}$. In addition, the hardware has to be vacuum compatible to $\sim 10^{-6}$ torr. Unlike ultra-high vacuum (UHV) requirements achieving this mid-level range of vacuum (HV) was not too difficult, for instance, holes were left blind tapped without vented hardware. The parts were cleaned and care was taken during assembly to avoid contaminating the surfaces. The assemblies were all fabricated in house at SLAC with the exception of the magnet blocks which were provided by an outside vendor.

The undulator was the first component designed and fabricated. It underwent several iterations to include first the motorization of the gap adjustment and later to add scintillator screens to a common base with the undulator. The holder assembly consists of several steel blocks and plates screwed together. The initial design had $\frac{1}{4}-80$ screws for gap adjustment, later replaced with stepper motors for the high harmonic scan experiment (chapter 4) and Linear Variable Differential Transformers (LVDTs) for position read back. Figure 3.5 shows the evolution of the undulator from the initial design, the motorized gap scan design, and the final configuration that adds the screens. All three designs also include screw adjustment of the height of the entire undulator assembly relative to the experiment table.

The chicane support structure was fabricated in copper instead of steel to aid heat flow from the coils to the water coiling in the base plate. The base plate also features screws for height adjustment. Figure 3.6 shows the chicane after fabrication. 


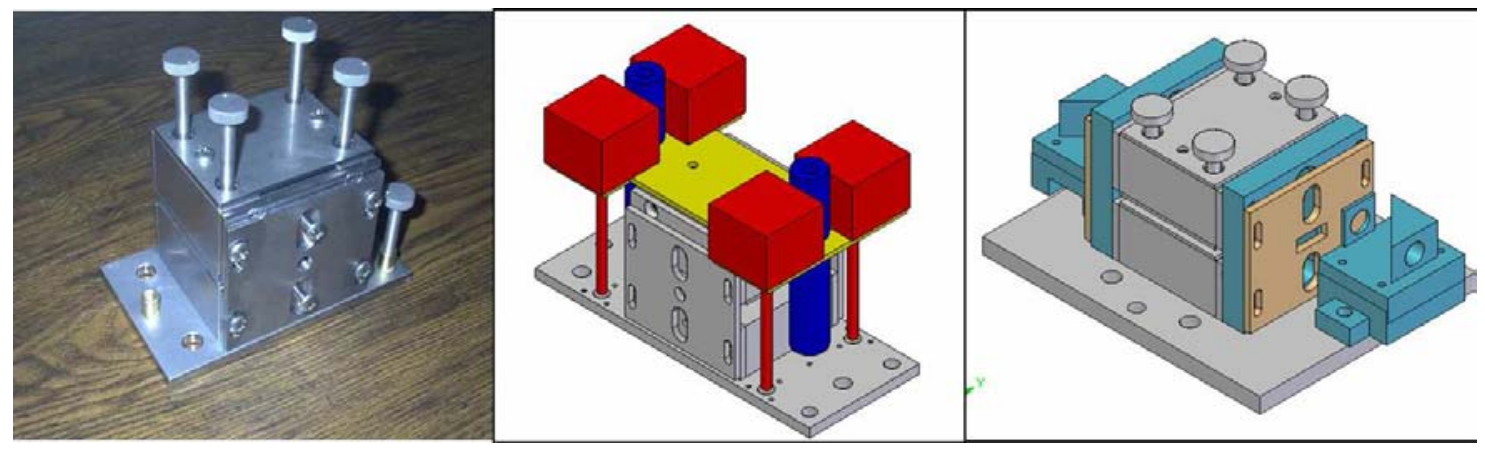

Figure 3.5: Progression of the undulator design. Left: Initial undulator design after fabrication. Center: CAD image of assembly used in the Harmonic IFEL experiment. The stepper motors are marked in red and the LVDTs in blue. Right: Final incarnation of the assembly used in the microbunching and net acceleration experiments.

\subsubsection{The Undulator Error and Correction}

After fabrication and assembly the undulator field was measured by a standard Hall probe scan. Scans revealed a design error in the tailoring of the end fields. The error produced a non-zeroed second field integral resulting in a potential beam side step of $340 \mu \mathrm{m}$, larger than the laser and electron spot sizes. It was eventually found that poor meshing of the shorting plate apertures led to a simulation error that showed a greater amount of field inside the apertures than in reality. In order to correct for the error, additional spacers were added that pushed the end plates out an additional $14 \mathrm{~mm}$. Figure 3.7 shows a plot of the $2^{\text {nd }}$ field integral before and after correction of the end plate separation. Additional smaller spacers of $0.2,0.5$, and $1 \mathrm{~mm}$ were used to fine tune the field integrals to zero.

The design error was actually turned into an advantage for the higher harmonics experiment (see chapter 4). The large spacers were removed to allow the beam side step. This enhanced coupling to even harmonics and also simplified alignment of the laser into vacuum since now the laser could travel through the undulator at an angle allowing an off-axis turning mirror to be used. 


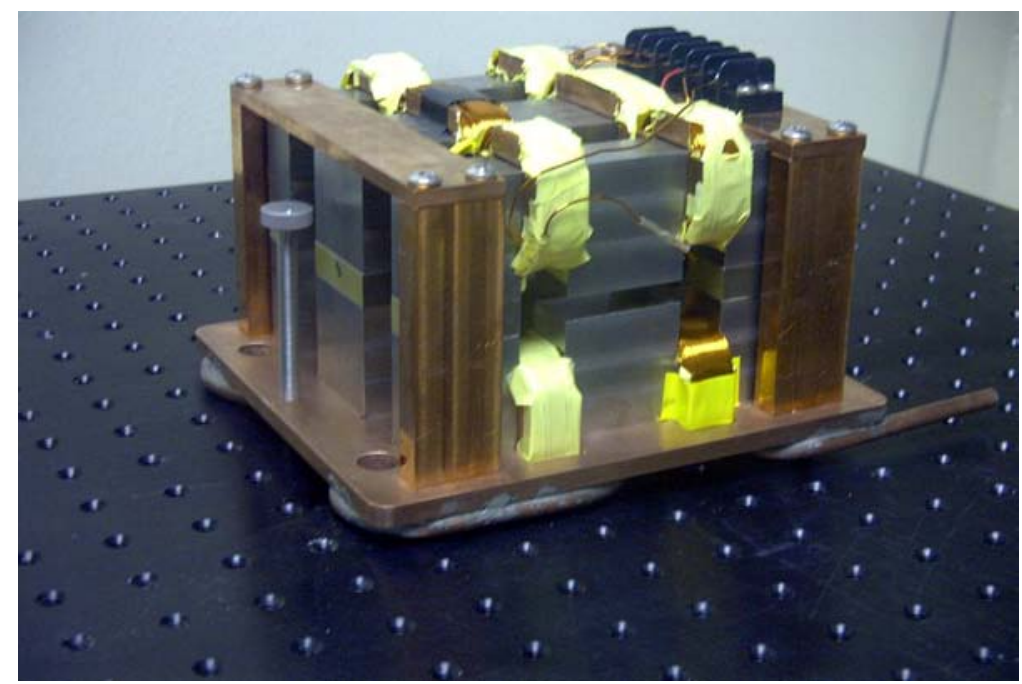

Figure 3.6: The chicane after fabrication. The coils are temporally held in place by tape (later removed).

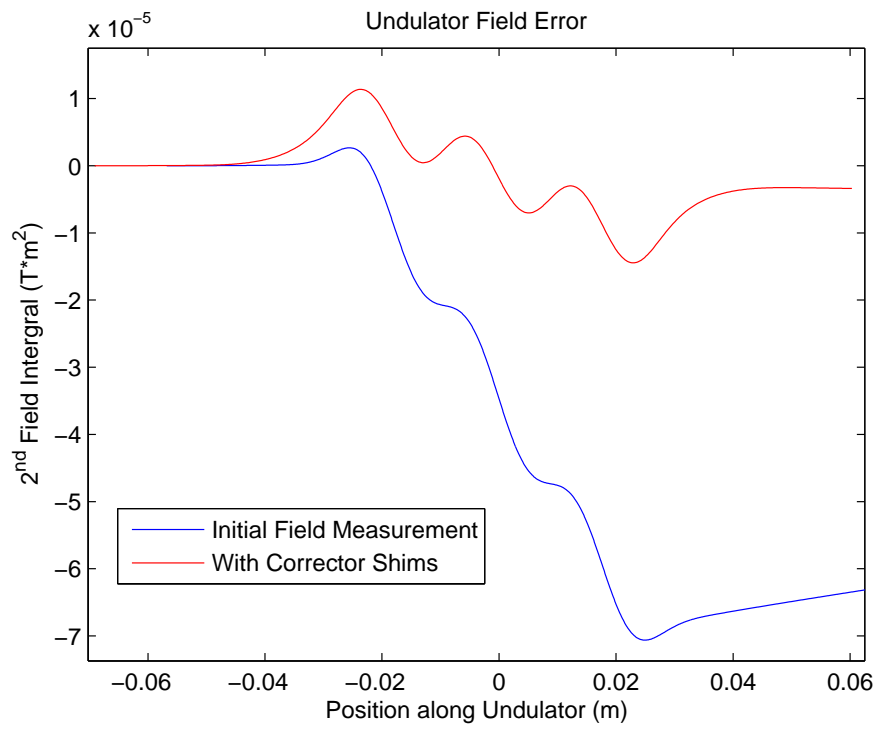

Figure 3.7: The $2^{\text {nd }}$ field integral through the undulator before (blue) and after (red) correction of the end plate spacings. For a $60 \mathrm{MeV}$, the initial side step due to the error corresponds to $340 \mu m$. 


\section{Chapter 4}

\section{The Higher Harmonics Experiment}

\subsection{Motivation for and description of the experi- ment}

Prior to the start of experiments at the new E163 facility at SLAC, the decision was made to do one last research run using the original LEAP setup at the Hansen Experimental Physics Labs's Superconducting Accelerator (HEPL-SCA). The primary purpose of this research run was to attempt the ITR experiment and its improvements described in $\S 1.2$. At the same time, however, we realized this would also serve as an opportunity to test the first component of the microbunching hardware: the IFEL.

The HEPL-SCA facility has a beam energy of $30 \mathrm{MeV}$, half that of the NLCTA for which the IFEL was originally designed. Although the undulator has an adjustable gap, that adjustment cannot accommodate halving the beam energy while maintaining resonance at the fundamental. However, the interaction can still occur at higher harmonics. Recall from $\$ 2.3$, rewriting the resonance equation in terms to find the harmonic number we have:

$$
n=\frac{\lambda_{w}}{2 \lambda_{l} \gamma^{2}}\left(1+\frac{K_{w}^{2}}{2}\right)
$$

where $K_{w}$ is the normalized magnetic field of the undulator, $\lambda_{w}$ the undulator period, $\lambda_{L}$ the laser wavelength, and $\mathrm{n}$ the harmonic number. Since the beam energy is half 
the undulator design value, for this experiment we have a starting harmonic number of 4. The gap adjustment allows a range of $K_{w}$ from 0.63 to 1.7, thus we should observe resonance from $n=4$ to $n=8$. An estimate for the relative strength of each of the resonances can be found using equation 2.28. We can define the normalized coupling coefficients $J J_{n}$, as:

$$
J J_{n}=\sum_{m=-\infty}^{\infty} J_{m}(n \xi)\left[J_{2 m+n+1}(n Z)+J_{2 m+n-1}(n Z)\right]
$$

For the higher harmonic experiment the electron and laser beams propagate at an angle in order to couple to even harmonics. This angle is chosen to be $\sim 15 \mathrm{mrad}$ for reasons described in the next section. The resulting coupling coefficients are plotted in figure 4.1.

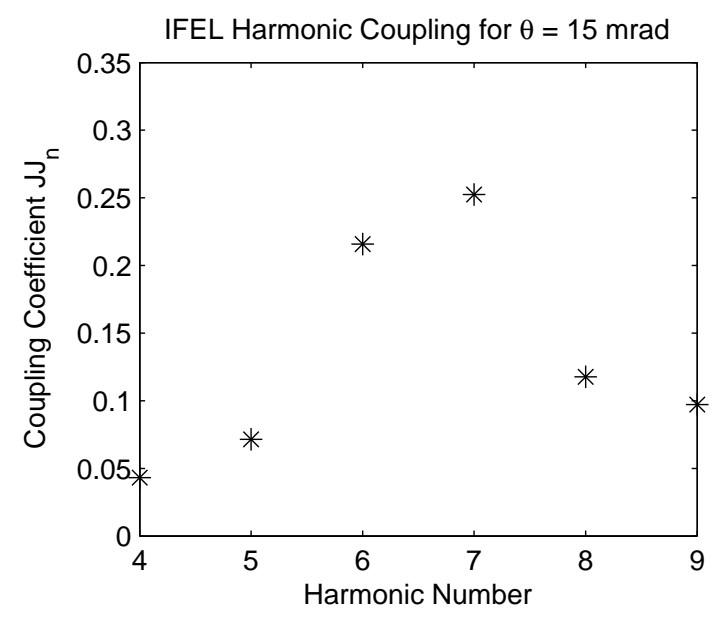

Figure 4.1: Coupling coefficients to each harmonic for the LEAP-IFEL experiment given by 2.28 .

We see from figure 4.1 that contrary to intuition, the higher harmonics are not necessarily weaker. To observe these higher harmonics, between data runs the gap height of the undulator is varied, thus changing $K_{w}$. It is expected that when $K_{w}$ satisfies equation 4.1 the laser-electron interaction will be strongest. Unfortunately, for comparing to experiment, equation 2.28 is of little use. In the real experiment the electron motion is much more complicated owing to the end fields of the short 
undulator. Both beams are also tightly focused in the middle of the undulator to maximize the interaction strength. Additionally, to avoid constant realignment, the laser is kept at the same trajectory between data runs. Meanwhile, as the gap is adjusted the electron trajectory changes due to changing field strength. As a result the overlap between laser and electron beam is optimal for only a small range of the scan and falls elsewhere. The net effect of all the complications is that it becomes best to compare the experimental data to simulation. A simple particle tracking code, described in section 2.5 is used for this purpose. Simulation results are shown in the plots along side the data.

Table 4.1 summarizes the parameters for the IFEL higher harmonic experiment. Several values were measured using the laser interaction itself, including the transverse and temporal sizes deduced from scanning the laser across the electron beam. The laser parameters were all measured prior to the experiment. The laser power was also monitored on a per shot basis and jittered by $5 \%$. The laser is synchronized to the accelerator with an additional phase shifter to allow scanning the laser past the e-beam in time. The shifter has a range of $>100 \mathrm{ps}$, and can be set between successive shots allowing for a randomly sampled correlation between the electron and laser beams to be generated. Both beams come to a focus in the middle of the undulator (figure 4.2). This increases the peak field of the laser necessary to obtain an appreciable interaction while maintaining good overlap. The beams are aligned using two phosphor (YAG) screens located at either end of the undulator.

The laser is introduced into the interaction chamber at an angle of $\sim 15$ mrad. This eliminates the need for a small chicane to pass the electrons around the last mirror inside the vacuum chamber or a pellicle which would need to be placed far from the focus to avoid laser damage. To maintain overlap of the electrons and laser inside the undulator along the full electron trajectory, the undulator end fields are de-tuned so that the electrons move with an angle similar to that of the laser (fig $4.2)$. 


\begin{tabular}{lc}
\hline Parameter & Value \\
\hline E-beam energy & $30 \mathrm{MeV}$ \\
E-beam initial energy spread (FWHM) & $30 \mathrm{keV}$ (typ.) \\
E-beam charge & $2 \mathrm{pC}$ \\
E-beam pulse length* (FWHM) & $\leq 1 \mathrm{ps}$ \\
E-beam normalized emittance & $2 \pi$-mm-mrad \\
E-beam focused vertical width* (FWHM) & $40 \mu \mathrm{m}$ (typ.) \\
E-beam focused horizontal width* (FWHM) & $210 \mu \mathrm{m}$ (typ.) \\
Laser pulse length (FWHM) & $2 \mathrm{ps}$ \\
Laser wavelength & $800 \mathrm{~nm}$ \\
Laser energy & $0.5 \mathrm{~mJ}$ \\
Laser focused spot size (FWHM) & $110 \mu \mathrm{m}$ \\
Undulator Period & $1.8 \mathrm{~cm}$ \\
Number of Periods & 3 \\
Strength $K_{w}$ (adjustable) & $0.63-1.7$ \\
\hline
\end{tabular}

Table 4.1: IFEL experimental parameters and parameters used in simulation. * Denotes values measured from the data itself. †The emittance and absolute charge were not measured during the experiment, but are nominal values for the HEPL accelerator.

\subsection{Experiment Results}

The data runs consist of several hundred laser-electron interactions taken at a rate of $10 \mathrm{~Hz}$. Energy spectra are recorded for each interaction. For each interaction the offset time between the electron beam and laser is randomly varied over a range of 20-30 picoseconds. In post-analysis, the widths of the energy profiles are calculated to determine the energy spread of the electron beam at each shot. Figure 4.3 shows an example scatter plot of the electron energy spread after the IFEL with the offset time between the two beams. The cross-correlation signal is clear. The width of the crosscorrelation compared to the known laser pulse length gives an e-beam length of $\sim 1$ ps. A least squares fit (solid curve) gives a mean interaction for ideal temporal overlap. A number of factors cause spreading of the data under the peak of the interaction; including temporal jitter and electron beam pulse length jitter. To factor out this additional spreading, the maximum interaction (dashed curve) is estimated from the strongest interactions of the peak. The amplitude of the maximum interaction is 


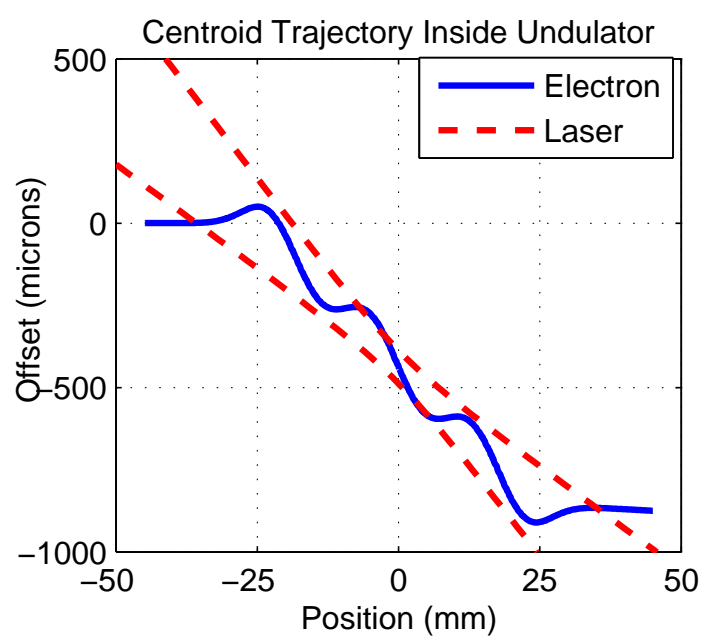

Figure 4.2: Calculated trajectory of beam from the measured fields of the undulator. The horizontal oscillation is $\sim 175 \mu \mathrm{m}$ peak-to-peak.

defined as the median of the top $10 \%$ of the data when the laser and electron are in time. Comparison between data runs with the same parameters has found that this peak interaction figure-of-merit has a factor of two better repeatability between runs compared to the least squares fit amplitude.

In addition to the time offset scan that occurs within every run, between runs other experimental parameters are varied to further explore the IFEL interaction. In particular, the transverse overlap is scanned using a mirror located far from the undulator. Also, to observe multiple resonances of the interaction, the gap of the undulator is varied from $4-11 \mathrm{~mm}$.

Results for the horizontal and vertical scans are shown in figure 4.4. While there are just a few runs for each scan, the data does shown good agreement with simulation. For each point corresponding to a single data run the maximal estimate is plotted against the laser transverse position. The laser waist is known from a knife edge measurement to be $110 \mu \mathrm{m}$ FWHM. The vertical overlap, which is a cross-correlation of the two waists, gives an estimate of the vertical electron beam size of $40 \mu \mathrm{m}$ FWHM. The horizontal overlap is wider than the vertical overlap due to the transverse oscillations of the electrons through the undulator. At $30 \mathrm{MeV}$ this oscillation is $\sim 175 \mu \mathrm{m}$ peak to peak (fig. 4.2). Comparison to simulation gives a horizontal spot 


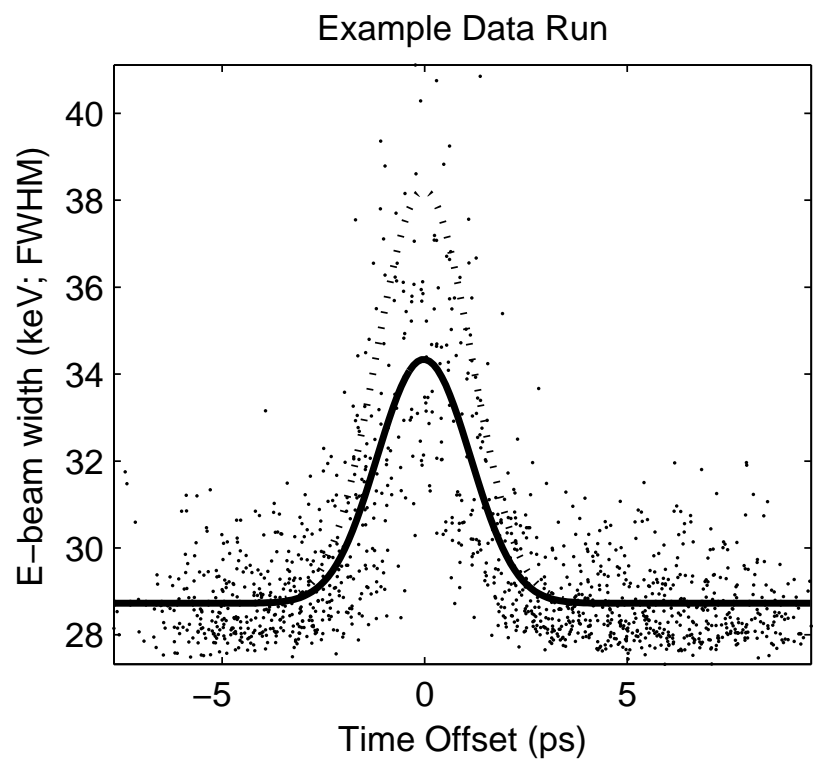

Figure 4.3: Example data run with 1500 laser on events. The solid curve is a least squares fit to all data points, and gives a mean interaction of $18 \mathrm{keV}$. The dashed curve is an estimate of the maximum interaction at ideal overlap, and gives peak interaction of $25 \mathrm{keV}$. The width of cross-correlation is $2.2 \mathrm{ps}$ rms.

size of $210 \mu \mathrm{m}$ FWHM. The asymmetric spot shape is confirmed qualitatively from observations of the spot shape noted at the time of the scan.

It is important to note that neither the data nor simulation in figure 4.4 has been rescaled in energy or offset, the simulation and experiment agree very well for these scans. Within the uncertainty of the runs, we find that $50 \mathrm{keV}$ is the maximum modulation seen for the IFEL interaction. If a chicane were included, this would give a bunching coefficient of $b_{1}=0.402$ where $b_{1}$ is the ratio of the first Fourier component of the longitudinal density to the average density (eqn. 2.32). The transverse overlap scans were done with the gap set to $6.3 \mathrm{~mm}$ which corresponds to the strongest resonance peak accessible by the experiment.

Compared to the transverse scans the gap scan interaction amplitudes (figure 4.5) are smaller by $50 \%$. The transverse overlap procedure is accurate to $25 \mu \mathrm{m}$ limited by the resolution of the camera observing the YAG screens, leaving $~ 10 \%$ uncertainty in the interaction amplitude. Also, there are a number of other parameters that can 

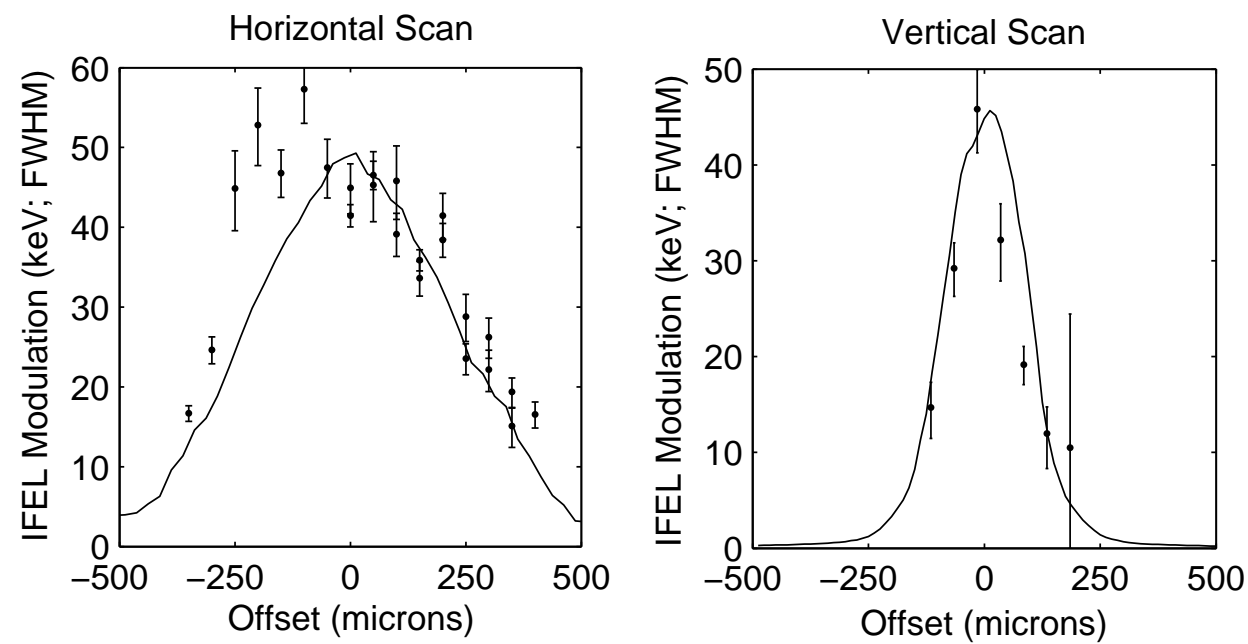

Figure 4.4: Vertical and horizontal overlap scans of the IFEL interaction. The solid curve is from simulation. The horizontal overlap is wider than the vertical due to the horizontal oscillation of the electrons within the undulator.

decrease the interaction amplitude including a larger transverse spot size, or longer electron pulse length. Clearly present in the data are two resonances, identified by comparison to equation 4.1 as the $5^{\text {th }}$, and $6^{\text {th }}$ resonances. The 4 th order resonance is also clear once it is presented alongside the simulation. While there are a number of data runs around the $4^{\text {th }}$ order peak with small or zero interaction seen, the mean of the data in the range $0.65-0.75$ is still well above zero and even slightly above the data from 0.75 to 0.9 indicating a peak. The $4^{\text {th }}$ order peak is also expected from equation 4.1. The simulation also shows additional resonances at larger $K_{w}$ values; the $7^{\text {th }}$ through $9^{\text {th }}$, however the data are too noisy to confirm their presence. While one might expect the 4th resonance to have a stronger interaction due to the lower order, there are two effects that change this. First, the coupling methods for even and odd harmonics are different, and with large crossing angles higher harmonics can actually have larger coupling strengths [40]. Second, since the alignment is not changed during the gap scan, as the gap increases the laser-electron overlap diminishes. The increase in gap decreases the magnetic field strength and therefore the electron horizontal motion decreases. This leads to a further roll-off of interaction intensity at large gaps and to a lesser extent as the gap becomes very small (the alignment was done at a 
gap of $\left.6.3 \mathrm{~mm} ; K_{w}=1\right)$.

Comparison to simulation is complicated by the fact that the overlap diagnostics do not give the absolute position of either beam with respect to the undulator. Therefore, the distance of the electron beam from the bottom pole tips is not well known. Since the field of an undulator varies as the hyperbolic cosine of the vertical position (eqn. 2.17), the field strength is in turn not well known. However, using the height of the beams as a free parameter in simulation, a best match can be found. Figure 4.5 gives the best match of simulation to the data where the height of the electrons off of the bottom pole tips is $2.5 \mathrm{~mm}$. The overall amplitude of the simulation is some $50 \%$ greater than the raw data, reaching a peak of $50 \mathrm{keV}$ on the 5 th harmonic in agreement with the interactions seen during the transverse scans.

With the inclusion of higher harmonics the IFEL can interact over a broad range of parameters. It is worth noting that the $5^{\text {th }}$ and $6^{\text {th }}$ harmonics are comparable in intensity; the IFEL interaction does not necessarily decrease with harmonic number. Both are in fact substantially stronger than the $4^{\text {th }}$ harmonic interaction. With an adjustment of the laser-electron angle the $4^{\text {th }}$ harmonic intensity could also be made stronger. This flexibility extends the utility of undulators or, more simply, aids the experimenter in changing other parameters such as choice of laser wavelength or beam energy.

\subsection{Implications for Advanced Accelerator Research}

This experiment has successfully observed harmonic interaction at the $4^{\text {th }}$ through $6^{\text {th }}$ order resonances from in IFEL with an operating wavelength suitable for efficient dielectric laser accelerators. The relative interaction peak amplitudes and spacing agree quite well with simulation. The harmonic interaction could be used in the future to improve bunching via a multicolor IFEL. 


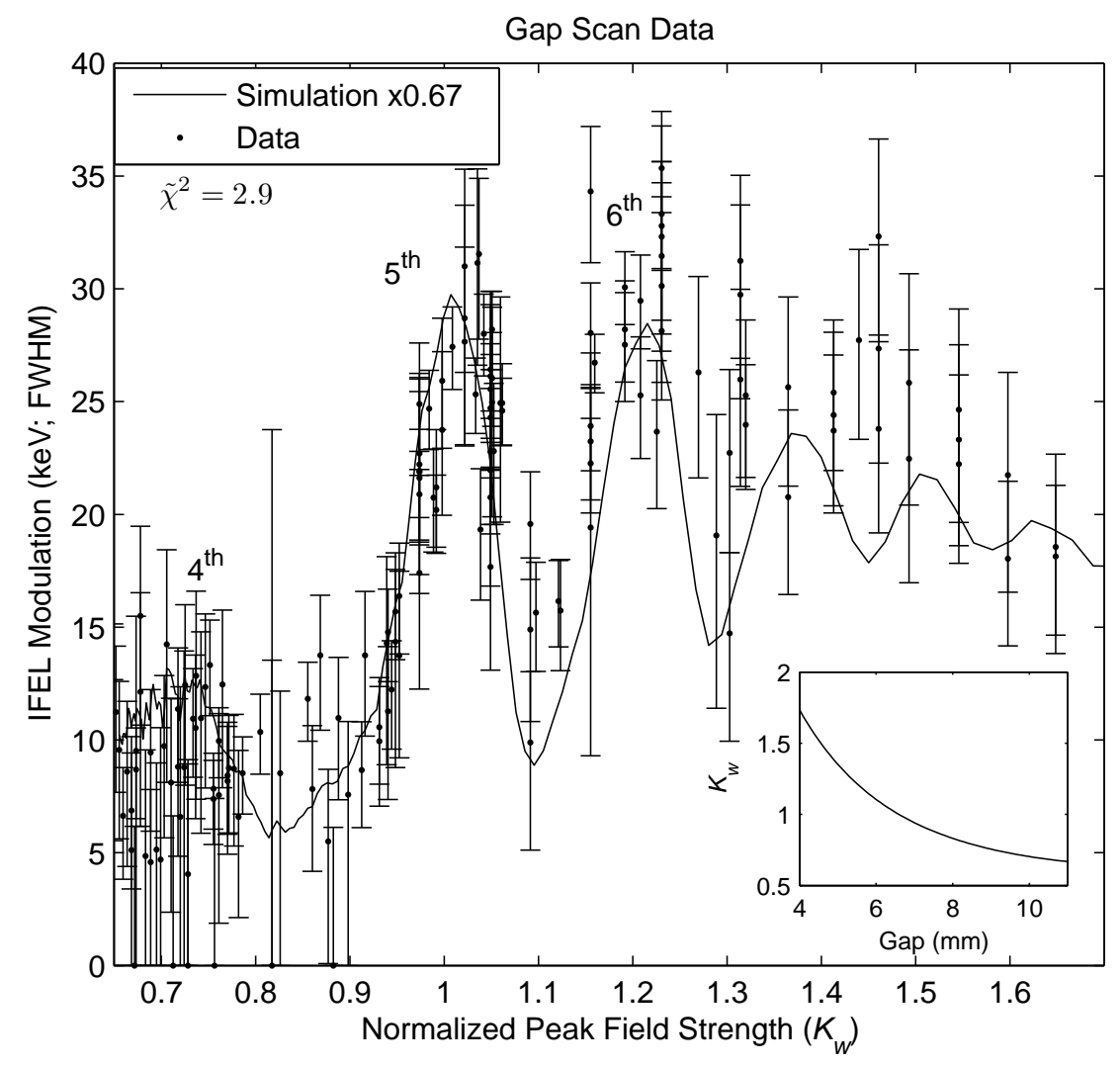

Figure 4.5: IFEL gap scan data, 164 runs total. Comparison to simulation (solid line) shows very good agreement to the shape and spacing of resonance peaks. The harmonic numbers are given next to each peak. Simulation has been rescaled vertically by 0.67 to better visualize overlap. The inset shows the normalized peak field strength as a function of the undulator gap. 


\section{Chapter 5}

\section{The E163/NLCTA Facility}

The E163 research program is hosted at the Next Linear Collider Test Accelerator (NLCTA) facility at SLAC. The NLCTA was originally built as a test platform for $\mathrm{X}$-band RF accelerator components for the Next Linear Collider project, a large scale research facility for high energy physics at the energy frontier. The test accelerator consisted of a DC gun with a thermionic cathode producing $\mu C$ macropulse trains, a 2-meter $60 \mathrm{MeV} \mathrm{X}$-band accelerator followed by a magnetic chicane, and several additional test stands which when installed with X-band accelerator structures could deliver beams up to $350 \mathrm{MeV}$. Starting in 2002, the facility was upgraded for laser acceleration research under the E163 program. The front end DC gun was replaced with an S-band RF photoinjector to produce single sub-ps electron pulses. A second concrete shielded hall was constructed adjacent to the NLCTA beamline to house the E163 experiments. A dogleg beam extraction line was installed after the NCLTA chicane to bring the beam to the experimental area.

This chapter gives an overview of the NLCTA/E163 research facility. It begins with a section on the laser room. Section 5.2 describes the upgraded NLCTA beamline including the UV optics for photo-electron production in the injector and relevant beam parameters. Section 5.3 describes the E163 experimental hall including diagnostics common to all planned experiments at E163. 


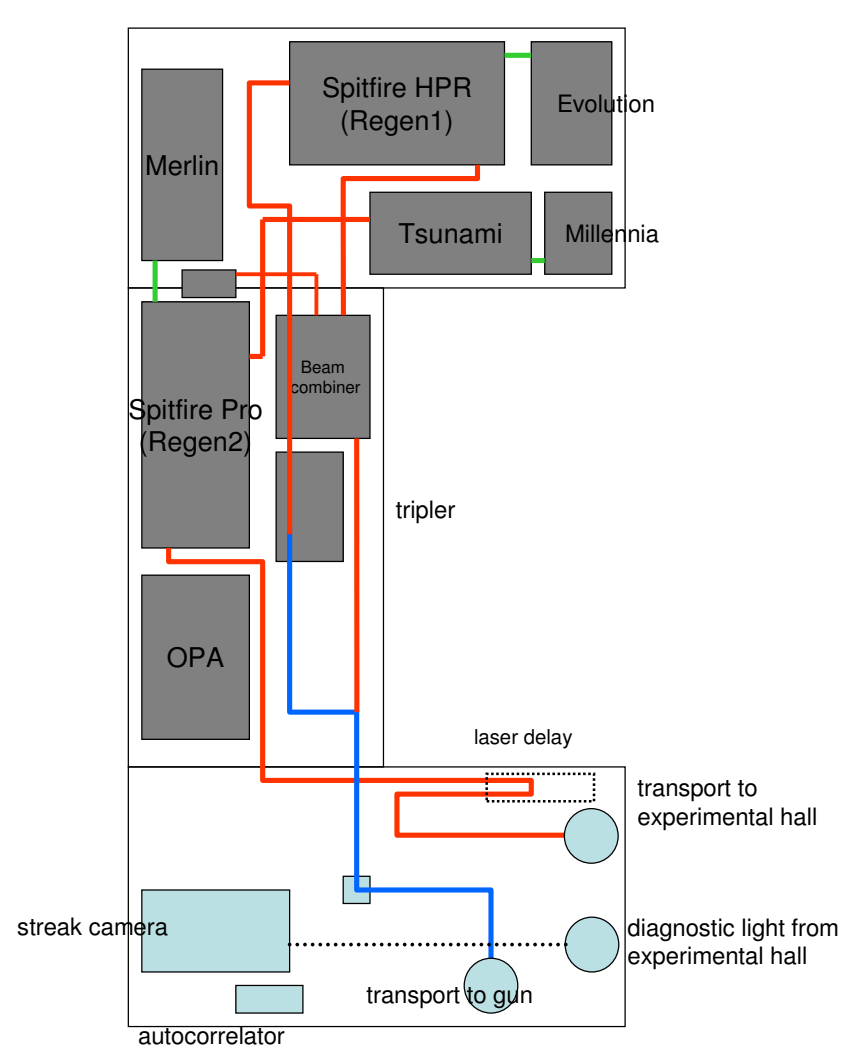

Figure 5.1: Layout of E163 laser system.

\subsection{The Laser Room}

The E163 laser room is a 500 sq. ft clean room located adjacent to both the NLCTA beamline and the new E163 experimental hall. It houses the lasers for driving both the photoinjector and the experiment, electronics for timing and communication with the main control program, a streak camera used in the experiments (described in further detail in §5.3), and space for laser only experiments.

A layout of the laser system is shown in figure 5.1. The laser system consists of two Ti:sapphire regenerative amplifiers (regens) driven by a common mode-locked Ti:sapphire oscillator. The oscillator is phase locked to a down-converted RF signal from the accelerator. This provides the necessary timing stability necessary to control 
the electron phase relative to the RF. The two amplifiers and oscillator each have their own pump laser. Table 5.1 lists the specifications of each of the lasers in the laser room. One regen (the Spitfire HPR, hereafter referred to as Regen1) is used to produce UV light by conversion to the third harmonic of the primary laser beam (266 nm). This is needed for efficient photoemission from the cathode surface. The UV laser light is combined with a CW IR beam and a portion of the light from the oscillator and is sent through an optical transport line to the RF gun optical table (see §5.2). The second regen (hereafter referred to as Regen2) is used for the experiment. The current setup sends the IR directly to the experimental hall along with a $\mathrm{CW}$ alignment beam. However, in future experiments the Regen2 will power an optical parametric amplifier (OPA), a tunable laser capable of producing picosecond IR pulses with a center wavelength from 1.1 to $3.0 \mu \mathrm{m}$.

The oscillator light is split three ways between the two regens and light sent to the gun optical table. The pockel cells for Regen2 are delayed by $\sim 200 \mathrm{~ns}$ with respect to Regen1 to account for the shorter path length to the experiment for the laser compared to the electron beam. The delay can be varied in steps of the oscillator period of $12.5 \mathrm{~ns}$, but can also vary by steps of regen round-trip time of $10 \mathrm{~ns}$. However the round-trip time is not a free parameter, instead being optimized on total laser power output. To adjust the delay below the $12.5 \mathrm{~ns}$ steps of the oscillator there is a trombone delay just before the transport pipe to the experimental hall. In addition, there is a fast delay stage in the path from the oscillator to the input of Regen2 with a range of $100 \mathrm{ps}$ and a settling time of $\sim 50 \mathrm{~ms}$. The delay stage is voice coil actuated and has an optical encoder for read-back. This is used during experiments to scan the laser past the electron beam in time forming a cross-correlation signal. 


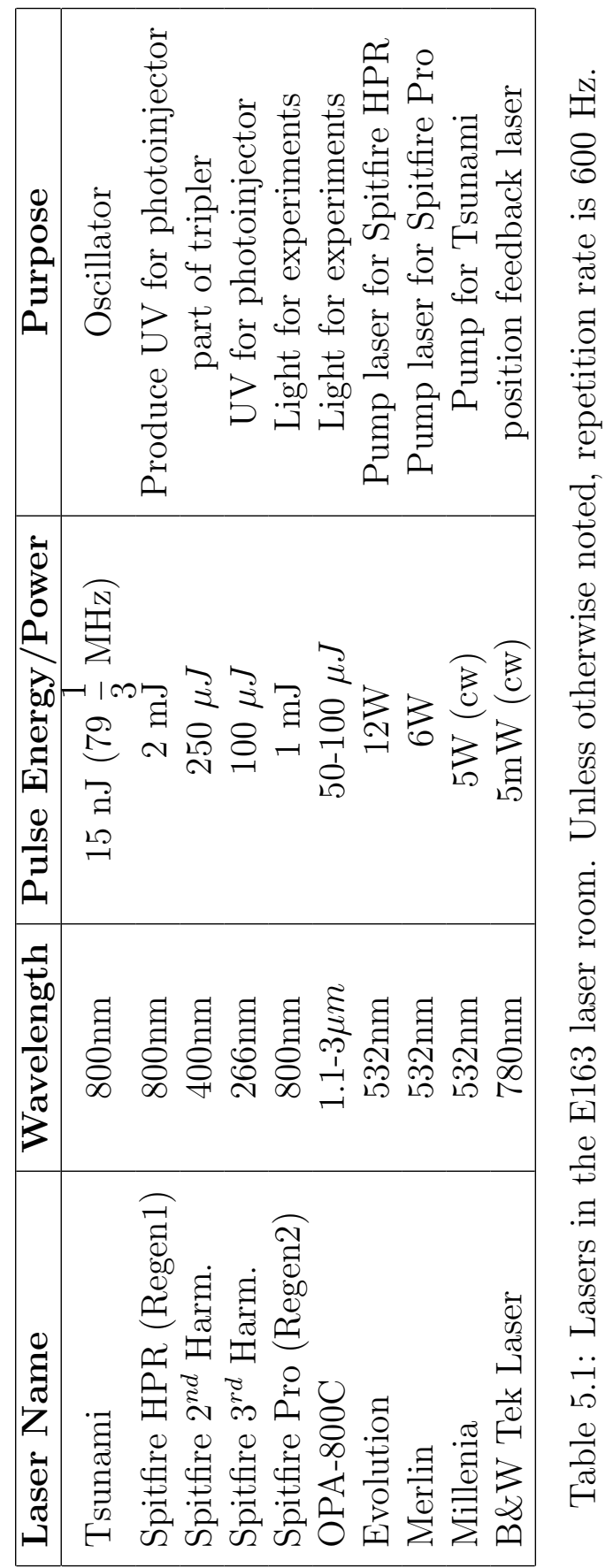


During experiments Regen2 is also randomly turned 'on' and 'off' during scanning to help distinguish true interaction signals from noise. The laser off data also aids in later analysis to study electron beam variations and drifts. The laser is turned on and off by manipulating the second pockel cell timing. In the 'on' state, the pockel cell timing is optimal and a single high power pulse is produced from the regenerative amplifier. In the 'off' state, the second pockel cell is delayed $\sim 64 \mathrm{~ns}$ with the result that most of the energy in the amplifier is lost before the pockel cell is triggered. There is also a small amount of leakage through the pockel cell even when not triggered, producing a string of low intensity IR pulses that go to the experiment. This leakage light is useful for alignment to the electron beam, but will not damage any of the in-vacuum screens when they are inserted. The laser on/off system is also used to step Regen 2 down to $10 \mathrm{~Hz}$ by only gating the laser on/off command signal with a $10 \mathrm{~Hz}$ trigger synchronized with the accelerator. Thus, although the regen is pumped at $600 \mathrm{~Hz}, 590$ of the shots will be in the 'off' state regardless of the on/off status. This is done to limit potential damage to in-vacuum experimental hardware.

The lasers are followed by focusing and combining optics. Each of the beams to the gun table are separately focused and then combined using a polarizing beamsplitter cube to combine the two IR beams and a cold mirror to combine the UV with the IR. For the lasers to the experimental hall, the beams are combined via a polarization beamsplitting cube.

To diagnose the lasers there is a fiber coupled spectrometer and numerous power meters. There is also a commercially made autocorrelator which is good for analyzing the oscillator as well as an in house assembled autocorrelator used for the two regens and the OPA. In the beam path of the UV to the gun is a fast steering mirror used for position feedback. This helps remove the effects of air currents and vibrations from the jitter of the laser spot at the cathode.

\subsection{The NLCTA beamline}

As mentioned in the prior section, UV light for the photocathode along with IR diagnostic light is brought to a $4^{\prime} \times 6^{\prime}$ optical table adjacent to the RF photocathode. 
The table includes diagnostics and matching optics to illuminate the cathode.

The gun optical diagnostics include a fast photodiode monitoring the $79 \mathrm{MHz}$ oscillator light and a quadrant detector for position stabilization of the optical transport. The $79 \mathrm{MHz}$ monitor helps diagnose potential timing jitter of the laser relative to the S-band RF system[48]. The laser position monitor is part of a feedback system that is located in the laser room. On the gun optical table there are also several cameras for aiding in optical alignment of the laser through the transport and also a 'virtual cathode' monitor that duplicates the laser spot that hits the cathode.

A key element in the gun table laser optics is the pulse front tilt of the laser beam[49]. The laser enters the RF gun through a side port that is $72^{\circ}$ from normal incidence. The transverse spot size of the laser on the cathode is $\sim 1$ millimeter. Without tilting the pulse front of the laser beam, this would result in a delay between one side of the laser hitting the cathode versus the opposite side of several picoseconds, much longer than is desired for the experiment. Furthermore, such a long electron pulse would pick-up a large energy spread in the following X-band accelerator where 1 ps equals $4^{\circ}$ of $\mathrm{X}$-band phase. A 5 ps long electron pulse centered on the crest of the acceleration would therefore gain an energy spread of $1-\cos \left(5 * 4^{\circ} / 2\right) \cong 1.5 \%$.

A layout of the optics to produce the pulse front tilt is shown in figure 5.2. A pair of prisms is used to give the UV laser its an initial tilt[50]. A pulse front tilt will occur in any system with angular dispersion such as the prisms[50]. For the pair of fused silica equilateral prisms used here, the tilt produced is $12^{\circ}$. A 6 -to- 1 horizontal compression using cylindrical lenses is used to increase the tilt to $72^{\circ}$ to match the incidence angle of the RF gun viewing port. The laser spot is incident on an iris that can be used to adjust the transverse spot size on the cathode. The distances from the prisms, through the 6-to- 1 telescope, to the iris are arranged so that the prisms are imaged on the iris. The imaging is needed to prevent chromatic dispersion from enlarging the spot size transversely. Without the imaging, the angular dispersion of the prisms combined with the laser bandwidth of $1 \mathrm{~nm}$ would induce a horizontal spread of $1.3 \mathrm{~mm}$, comparable to the spot size after telescoping and enough to wash out the pulse front tilt. The iris is then again relay imaged 1-to-1 to the cathode surface. A pick-off just before the cathode sends a small amount of light to the 
virtual cathode monitor.

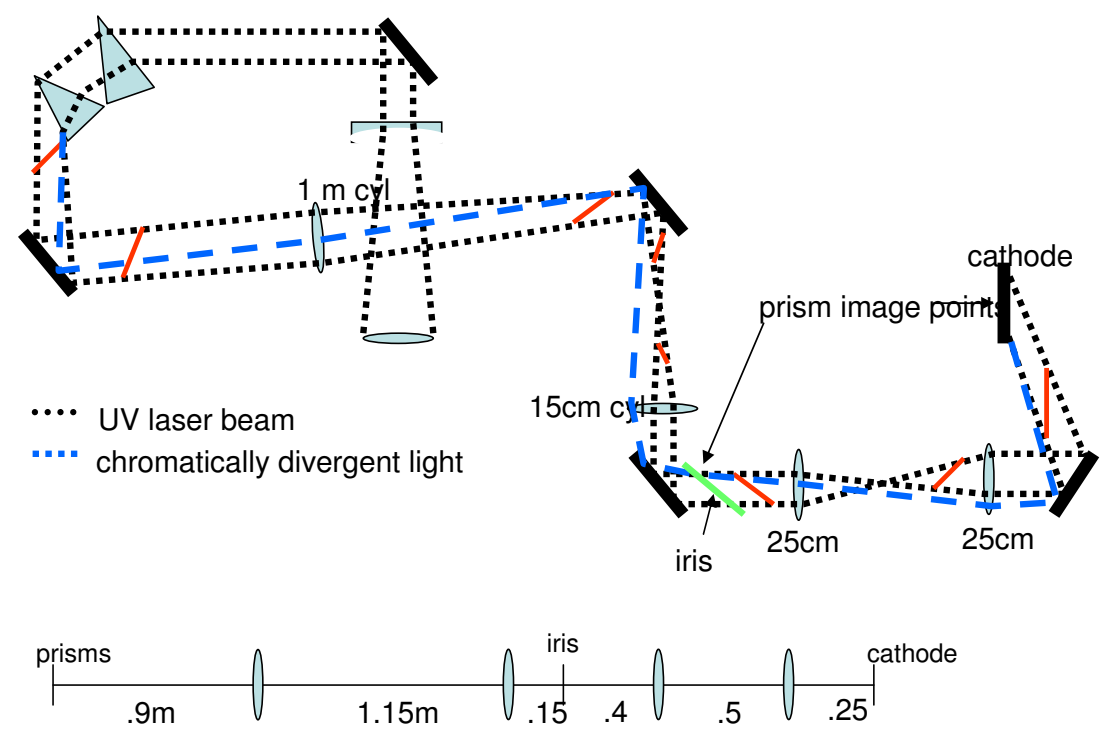

Figure 5.2: Layout of the pulse front tilt. The red lines indicate the progression of the pulse front tilt.

A block diagram of the NLCTA is shown in figure 5.3. The RF gun is a one and half cell accelerator with a gradient of $\sim 100 \mathrm{MeV} / \mathrm{m}[51,52,53]$. This strong acceleration gradient helps combat space charge growth of the beam right after emission from the cathode. The cathode is poly-crystalline copper. The laser is timed such that electrons are emitted $60^{\circ}$ off of crest, but slip in phase during acceleration such that they reach peak phase at the end of the gun. At the exit of the gun the beam has an energy of $5 \mathrm{MeV}$. The gun is surrounded by a strong solenoid that refocuses the beam into the X-band accelerator negating the space charge expansion force[54]. Between the gun and the $\mathrm{X}$-band accelerator there is a $\sim 1 m$ drift that houses diagnostics for the gun. This includes two combined function scintillator screens - Faraday cups. There is also an adjustable $70^{\circ}$ dispersive bend with its own scintillator/Faraday cup for viewing the beam energy from the gun. This allows optimizing the phase of the laser to S-band. 


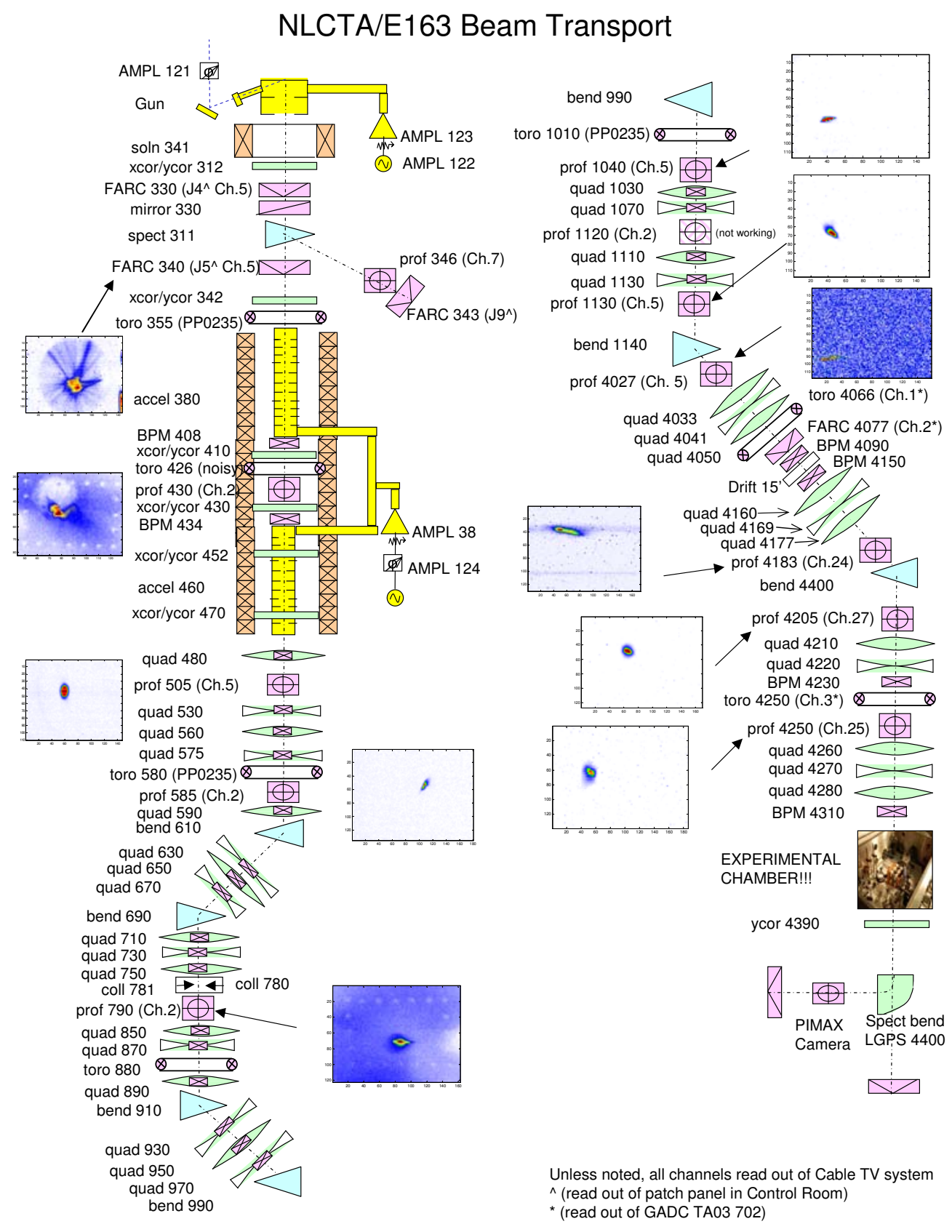

Figure 5.3: Block diagram of the NLCTA/E163 accelerator beamline. Shown are all quadrupoles and steerers, along with diagnostics (toroids, profile screens). NLCTA Layout diagram by Chris McGuinness (C). 
The X-band accelerator consists of two 1-meter sections each delivering up to $30 \mathrm{MeV}$ energy gain. These accelerator sections are powered from a single X-band klystron. The RF pulse out of the klystron is compressed by the SLED-II system[55] which uses a $\sim 50 \mathrm{~m}$ long delay line to store energy until released by a phase flip in the incoming RF. The delay line is highly sensitive to thermal drifts making necessary a feedback system to maintain a good quality RF output pulse from the SLED system. There are also feedback systems on both the X-band klystron amplitude and phase[48]. Table 5.2 lists nominal beam parameters for the NLCTA accelerator.

\begin{tabular}{|l|c|}
\hline Parameter & Nominal \\
\hline Charge & $50 \mathrm{pC}^{*}$ \\
Energy after photoinjector & $5 \mathrm{MeV}$ \\
Final Energy & $60 \mathrm{MeV}$ \\
Energy Spread & $0.1 \%$ \\
Emittance & $1 \mathrm{~mm}-\mathrm{mrad}$ \\
Pulse Length & $1 \mathrm{ps}$ \\
\hline
\end{tabular}

Table 5.2: List of nominal beam parameters for the E-163/NLCTA beamline. *A maximum of $450 \mathrm{pC}$ has been produced from the photoinjector with optimum UV tuning, however administrative controls limit charge to the experiment at $50 \mathrm{pC}$.

The accelerator is followed by a matching section with four quadrupoles and two profile monitors. The beam then goes into a dispersive chicane. Quadrupoles along the chicane are set to give a focus in the middle of chicane while still leaving the beam dispersed in energy. A profile screen in the center of the chicane allows setting of the X-band amplitude and phase. There is also a collimator close to the center of the chicane just before the screen to allow filtering of the beam energy.

The extractor bend and dogleg are located after another matching section following the chicane. The bend angle is $25^{\circ}$. The dogleg has two sets of three quadrupoles placed symmetrically about the midpoint of the dogleg. A set of three quadrupoles (called a triplet) allows full control of focus of the beam in both dimensions. Unlike the chicane, the dogleg quadrupoles are adjusted to give zero dispersion in the experimental hall. In the experimental hall the beamline consists of a short straight section with a doublet quadrupole pair and a final triplet used to focusing the beam into the experimental chamber. There are also a few more diagnostic cameras and toroids. 
All of the quadrupoles within the original NLCTA have installed dipole corrector steerers and beam position monitors (BPMs). Within the newer beamline including the dogleg and the line in the experimental hall there are only four BPMs installed at key locations between quadrupoles. Two are located symmetrically about the midpoint of the dogleg. The other two are on the straight section: one between the doublet and triplet, and the other just before the experimental chamber. In total there are 35 quadrupoles, $46 \mathrm{X} / \mathrm{Y}$ corrector pairs, $28 \mathrm{BPMs}$, and 12 profile screens along the beam line before the experiment. This makes for a highly versatile, easily adjustable, and well diagnosed beamline. At the same time, it presents a challenge in determining which magnets to adjust when needed. The initial commissioning of the E163 program was proceeded by a longer than expected learning period in tuning the beamline while at same time uncovering and removing bugs in the new hardware. Efforts were made to match beam observations and measurements to modeling of the beamline, and they remain on-going. Nevertheless, the experiments were able to proceed in parallel with learning how to correctly tune and run the beamline.

\subsubsection{Beam Characterization}

Characterization of the beam produced by the upgraded NLCTA facility remains an on-going process. The optimum transport and running methodology continues to be refined. As a result, the electron beam properties used during experiments change almost day to day. The electron beam also inherits many of the properties of the UV laser spot illuminating the cathode which is also not a constant. Nevertheless, there are a few measurements of the beam properties that are worth noting. In particular some quadrupole scans[56] for emittance have been done using quadrupoles 480 and 530 and a profile screen 585 just after the X-band accelerator sections (see figure 5.3). Figure 5.4 shows a pair of quadrupole scans for the nominal beam tuning out of the Xband linac. From the scans we see that the NLCTA accelerator is capable of producing beams with quite small transverse emittances, around $1 \mathrm{~mm}-$ mrad normalized. Less well known is the emittance further down the transport and into the experiment. Synchrotron radiation and focusing aberrations could enlarge the emittance prior to 
the experiment. Additional quadrupole scans of the beam entering the experimental chamber are needed to quantify this.
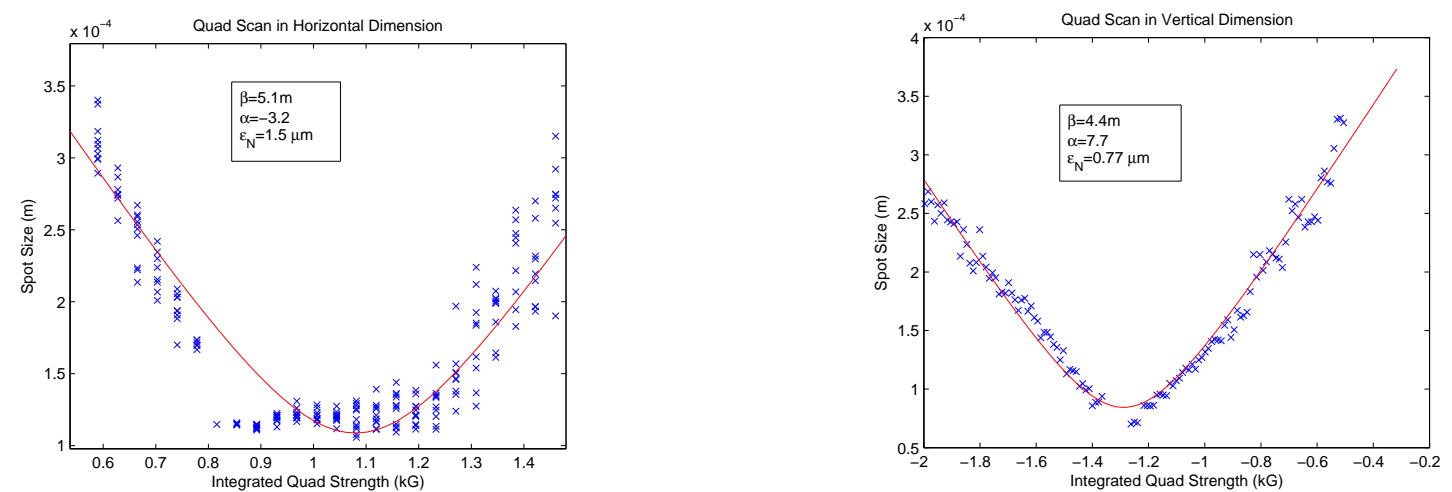

Figure 5.4: Quadrupole scans of the beam after the X-band accelerators.

As mentioned, one important design aspect of the transport lattice is control of the dispersion. In the middle of the NLCTA chicane, the dispersion should be large while at the same time the beam betatron envelope is minimized. This allows the collimator to effectively select a narrow energy spread. In the dogleg the dispersion must go through a minimum in the middle of the dogleg such that by straight section in the experimental hall, the dispersion is again zero. Elegant[43] based simulations were used to find an initial magnet configuration to accomplish this. However, inevitably there are uncertainties in the simulation versus real-world conditions. In particular, it is known that the coupler sections of the accelerators have some focusing that is not accounted for in the simulations. It was therefore important to measure the dispersion of the beam along the transport. This was done by adjusting the X-band gradient and recording the position shift of the beam on the BPMs along the transport. If the dispersion is zero at a given BPM, there will be no centroid motion. Figure 5.5 shows an example of a dispersion scan of the NLCTA-E163 beamline. Each point on the figure represents a linear fit to a correlation of BPM position to X-band amplitude. The X-band amplitude is typically varied $1-2 \%$ during a scan with 30 samplings of the amplitude and 5 BPM samples per amplitude setting. 


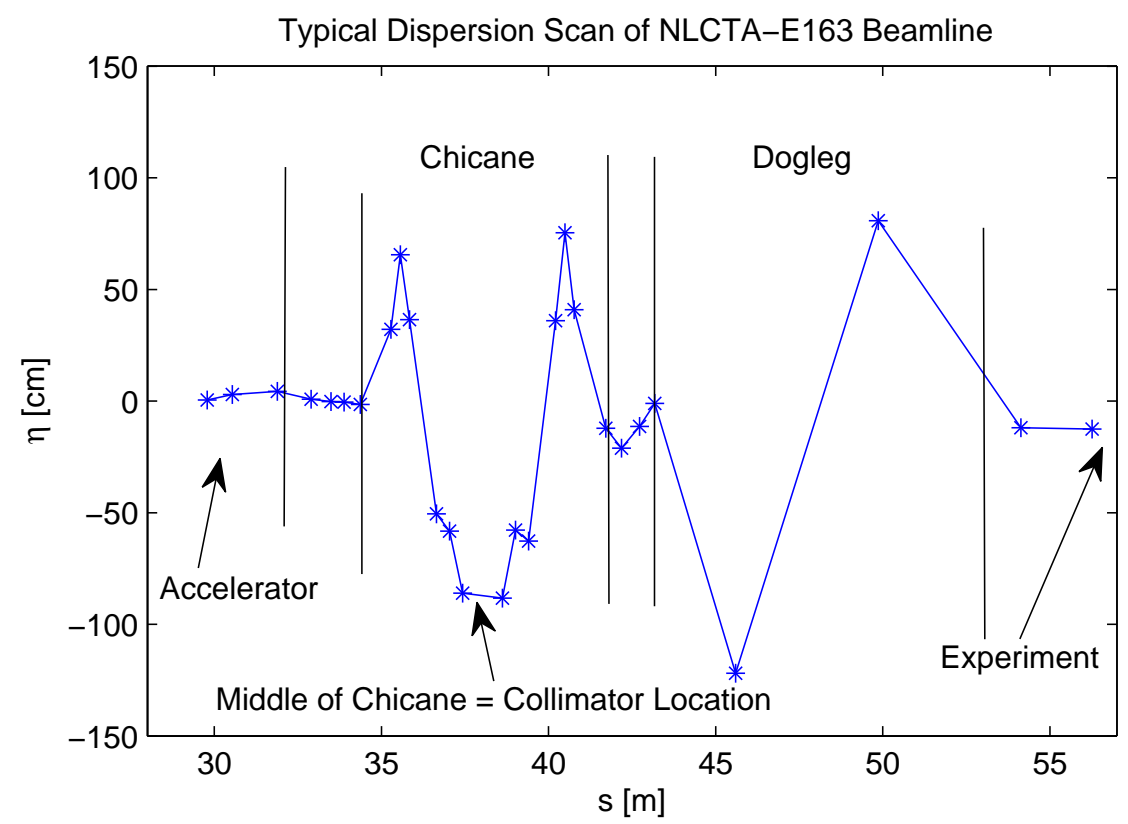

Figure 5.5: A typical scan of the dispersion of the NLCTA-E163 beamline. Each point represents a linear fit to a correlation of BPM position to X-band amplitude.

\subsection{The E163 Experimental Hall}

The E163 Experimental hall is a $20^{\prime} \times 35^{\prime}$ shielded room adjacent to the NLCTA accelerator. The beam transport brings the electron beam to a $3^{\prime} \times 1.5^{\prime} \times 2^{\prime}$ vacuum chamber which houses the experiments. The chamber has numerous vacuum ports for diagnostics, laser input, spy cameras, and electrical feedthroughs. The chamber sits atop a $4^{\prime} \times 6^{\prime}$ optical table. On the table are cameras for viewing into the chamber and optics for steering and focusing the laser into the experiment. There is also a smaller $1^{\prime} \times 3^{\prime}$ optical table next to the second dogleg bend for introducing laser light into a viewport at the dipole bend.

Two pipes come from the laser room next door. One brings in the laser from the Regen 2 for the experiments. The second pipe is used to send diagnostic light back to the laser room for analysis using the streak camera[48]. This diagnostic light is a combination of Cherenkov light produced from the electron beam passing through a $1 \mathrm{~cm}$ Aerogel sample located inside the experimental chamber and laser light that 
is sent into the experimental chamber roughly parallel with the electron beam and scatters off the aerogel. The aerogel is contained in a vacuum tight cell that is inserted and removed by a pneumatic actuator. Collecting optics transport the light out of vacuum and along the diagnostic line. The Cherenkov cell is re-imaged at the streak camera entrance slit. Optical attenuators are used to limit the laser power and avoid damaging the streak camera. The streak camera allows timing overlap to within about 50ps limited by jitter of the streak camera trigger. This level of accuracy is sufficient since the scan range of the fast delay is 100ps.

In addition to the aerogel cell there are a number of other diagnostics in the experimental hall that are common to all experimental setups. Diagnosing the laser are two photodiodes and a quadrant detector. The quadrant detector monitors transverse jitter of the laser. Even without active feedback on the laser position, measurements with the quadrant detector have found the jitter to be less than one quarter of the laser spot size. One photodiode is a large area diode directly illuminated by a $\sim 1 \%$ leak through of the IR light from the first mirror on the experimental table. The second diode is a faster, smaller area diode with a $1 \mathrm{~ns}$ rise time that monitors stray light off of a mirror. Both diode signals are sent to SRS boxcar integrators. The slow diode is used to monitor total laser power going to the experiment. The fast diode is setup with a small, 10ns, integrating window and is used to look for a common timing jitter of the laser system where, due to trigger jitters, the regen passes and amplifies an oscillator pulse that is one pulse earlier or later than the nominal one. Such a pulse will still have high pulse power but will fall outside the fast photodiode integration window.

The primary electron beam diagnostic is the $90^{\circ}$ energy spectrometer magnet. The spectrometer magnet disperses the beam in energy while at the same time in the horizontal dimension images the interaction point inside the chamber to a 1" scintillator crystal after the bend. This crystal is then viewed by a camera to measure the energy profile of the beam. The resolution of the energy spectrometer is about $5 \mathrm{keV}$ assuming an electron spot size of $50 \mu \mathrm{m}$. It has a field of view of $1.5 \mathrm{MeV}$, though typical beam energy spreads are only $30 \mathrm{keV}$. Future experiments could modulate the beam to several hundred $\mathrm{keV}$. The scintillator crystal is viewed by a Princeton Instruments 
PIMAX camera[57]; a 16-bit gated intensified camera that is linked directly to the main data acquisition computer.

A toroid located just upstream of the experimental chamber is also used during experiments to monitor the electron beam. This monitor serves as a charge measurement. Like the fast photodiode, the toroid has a fast response time which allows for a narrow time integration window on the boxcar integrator. This allows for a 'bucket-hopping' diagnostic on Regen1. Bucket-hopping is when trigger jitter causes the Regen's pockel cells to fire slightly later or earlier producing a laser pulse that is one round-trip time later or earlier. The round trip time of both lasers is $\sim 10 \mathrm{~ns}$.

\subsubsection{Laser/Electron Alignment}

An important step common to all the laser-electron acceleration experiments is the alignment of the laser to the electron beam. This alignment is done using screens inside the experiment chamber that are inserted into the beam path by pneumatic actuators. There are currently three such screens in use for the experiments: two are located immediately upstream and downstream of the undulator and a third is located just downstream of the image point of the spectrometer. The screens are made of Cerium doped YAG scintillator to show the electron beam. The Ce:YAG crystals are also roughened using a coarse polishing grit so that the screens will uniformly scatter the laser light. Since both beams are focused to small spots (typically $<100 \mu m$ ) a small field of view with good optical resolution is needed for alignment. For this purpose, the cameras are installed with long-work distance microscope objectives that have a working distance of $0.3-0.5 \mathrm{~m}$, and magnification of $\sim 1$ allowing the camera and optics to sit outside of vacuum while achieving the necessary image resolution.

Since the experiment uses a high peak power laser focused to a small spot size, the screens would be damaged if inserted while the laser was at full power. To avoid this, an interlock system is installed that monitors the screen position and prevents full power when a screen is insert. The interlock system is included in the laser on/off system described in $\S 5.1$ and sets the state to 'off' when a screen is in.

Even with the attenuation provided by the laser 'on/off' system, the laser light is 
still far too bright to produce useful image on the overlap monitors. There is therefore an additional ND5 attenuator that can be inserted by motorized optical flip mount (aka 'flipper'). 


\section{Chapter 6}

\section{Microbunch Formation and Characterization}

The first experiment upon commissioning of the E163 facility was the production and characterization of optical microbunches. The experiment took place in parallel with the continued refinement of beam tuning and running methodology and often more time was spent debugging new beamline components or repairing failures than collecting data. Nevertheless, after several months of effort a good, stable beam running condition was found and a complete set of data characterizing microbunching was obtained.

This chapter gives the results of the microbunching formation and characterization experiment. The chapter begins with a theoretical explanation for Coherent Optical Transition Radiation (COTR), necessary for characterizing the microbunches. Section

6.2 discusses the experimental layout and analysis methods. The remainder of the chapter discusses various aspects of the data itself. 


\subsection{Microbunch Characterization using Transition Radiation}

If the radiation pattern produced by a particle in some physical process is not a function of the particle position, as is the case for transition radiation from an infinitely wide foil, the total radiation from a collection of particles takes the form[58]:

$$
\begin{aligned}
\frac{d I_{\text {tot }}}{d \Omega d \omega} & =N\left[1+N|f(\vec{k}, \omega)|^{2}\right] \frac{d I_{0}}{d \Omega d \omega} \\
f(\vec{k}, \omega) & =\frac{1}{\rho_{0}} \int d \vec{x} d t \rho(\vec{x}, t) e^{i(k \cdot \vec{x}-\omega t)}
\end{aligned}
$$

Here, $I_{0}$ is the radiation pattern for a single particle, $N$ is the number of particles, $f$ is called the form factor equal to the normalized Fourier transform of the bunch density, and $\rho$ is the bunch charge distribution. Thus we have two terms: the first term, linear in the charge, gives the incoherent radiation contribution while the second, proportional to the square of the charge, is the coherent term. Studying the amount of coherent transition radiation therefore informs on the structure of the beam through $f$. For our microbunched beam, the charge distribution is given by:

$$
\begin{array}{r}
\rho(\vec{x}, t)=\frac{\rho_{0}}{\pi^{3} / 2 \sigma_{z} \sigma_{r}^{2}} e^{-\frac{r^{2}}{2 \sigma_{r}^{2}} e^{-\frac{(z-\beta c t)^{2}}{2 \sigma_{z}^{2}}}}\left\{1+2 \sum_{n=1}^{\infty} b_{n} \cos \left[n k_{L}(z-\beta c t)\right]\right\} \\
b_{n}=J_{n}\left(n k_{L} R_{56} \frac{\eta}{\gamma_{0}}\right) e^{-\frac{1}{2}\left(n k_{l} R_{56} \frac{\sigma_{\gamma}}{\gamma_{0}}\right)^{2}}
\end{array}
$$

Note that $\rho$ is the combination of a term describing the transverse Gaussian distribution and the longitudinal density modulation given in chapter 2, equation 2.31, describing the microbunching modulation. $b_{n}$ is known as the bunching factor at harmonic $n$ of the laser wavelength $=2 \pi / k_{L}$. We have also added the term $z-\beta c t$ to denote the temporal dependence of the bunch traveling at a speed $\beta c$. Finally, we have also included a term describing the longitudinal bunch envelope. To obtain $f$ we must take the Fourier transform of eqn. 6.3. This is best done by taking advantage 
of the Convolution Theorem, $\digamma(f g)=\digamma(f) \otimes \digamma(g)$ where $\otimes$ denotes convolution and $\digamma$ the Fourier transform. Using this fact we obtain:

$$
f(\vec{k}, \omega)=e^{-\frac{1}{2} k_{r}^{2} \sigma_{r}^{2}}\left\{e^{-\frac{1}{2}\left(\frac{\omega \sigma_{z}}{\beta c}\right)^{2}}+2 \sum_{n=1}^{\infty} b_{n} \exp \left[-\frac{1}{2}\left(\frac{\omega}{\beta c}-n k_{L}\right)^{2} \sigma_{z}^{2}\right]\right\}
$$

Here $k=\omega / c$ is the wavenumber and $k_{r}=k \sin \theta$ is the transverse component of the wavenumber with $\theta$ the polar angle of emission. We can see from equation 6.5 that the microbunch train produces coherent radiation not only at the fundamental laser wavelength used to produce the microbunches but also at harmonics. The DC portion of the microbunching density convolved with the finite pulse envelope produces a long wavelength terahertz pulse. At the bunching harmonics the COTR amplitude is directly related to the bunching factor at that harmonic. The COTR can therefore be used to measure properties of the bunches. The COTR at each harmonic also has a narrow bandwidth due to the finite pulse length of the beam. For the parameters of this experiment, $\sigma_{z}=0.3 \mathrm{~mm}$ and $\lambda_{L}=.8 \mu \mathrm{m}$ so that the bandwidth is $0.3 \%$ or $2 \mathrm{~nm}$.

In order to estimate the total energy radiated coherently into each harmonic we must know $I_{0}$. At relativistic energies, transition radiation for a single particle is given by the Frank-Ginsburg formula[59]. This forms a cone with a characteristic opening angle of $\theta=1 / \gamma$.

$$
\frac{d I_{T R}}{d \Omega d \omega} \cong \frac{e^{2}}{4 \pi^{3} \varepsilon_{0} c} \frac{\theta^{2}}{\left(\frac{1}{\gamma^{2}}+\theta^{2}\right)^{2}}
$$

Equation 6.6 assumes an ideal conductor which at optical frequencies is a valid approximation. Notice that equation 6.6 has no frequency dependence, thus the frequency dependence of COTR is entirely determined by the bunch form factor $f$. Combining equations 6.6, 6.5, and 6.1 and integrating over frequency we can obtain an expression for the total COTR yield at each harmonic $n$. 


$$
\frac{d I_{C O T R, n}}{d \theta} \cong \frac{N^{2} e^{2} b_{n}^{2}}{4 \pi \sqrt{2 \pi} \varepsilon_{0} \sigma_{z}} \frac{\theta^{3}}{\left(1 / \gamma^{2}+\theta^{2}\right)^{2}} \exp \left[-\left(k \sigma_{r} \theta\right)^{2}\right]
$$

Where now $k=k_{L} n, k_{L}$ is the wavenumber of the laser. We have also taken $d \Omega=\sin \theta d \theta d \phi$ and integrated over $\phi$. For relativistic beams $1 / \gamma \ll 1$ meaning that the radiation is strongly peaked at a small angle $\theta$. This allows the additional approximation $\sin \theta \approx \theta$. Equation 6.7 shows that the transverse profile of the radiation pattern is modified from the single particle pattern by the transverse form factor. We can see this more explicitly by plotting eq. 6.7 for two values of $k \sigma_{r}$ (figure 6.1). For small values of $k \sigma_{r}$ the pattern is the same as the pattern from a single particle. However, when $k \sigma_{r} \gtrsim \gamma$ the transverse term suppresses the radiation pattern for larger $\theta$, making the outgoing radiation pattern sharper, but more importantly decreasing the total yield.

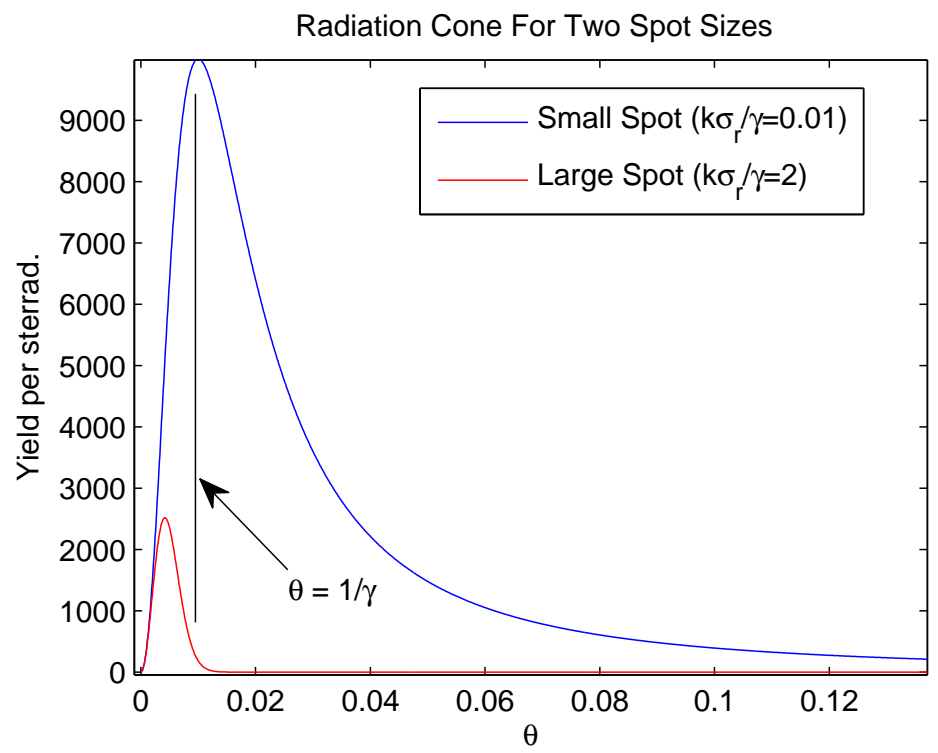

Figure 6.1: The COTR angular distribution for two values of $k \sigma_{r}$ and $\gamma=100$.

To calculate the total yield we must integrate equation 6.7. We can obtain two analytic solutions for asymptotic behavior. For small spot sizes we can ignore the transverse form factor and use the solution for a single particle. In the limit $k \sigma_{r} \gg \gamma$ we obtain a second limit derived in [60]. 


$$
\begin{array}{ll}
I_{C O T R, n} \propto 4 \ln \gamma-1 & k \sigma_{r} \ll \gamma \\
I_{C O T R, n} \propto\left(\frac{\gamma}{k \sigma_{r}}\right)^{4} & k \sigma_{r} \gg \gamma
\end{array}
$$

For small spot sizes where the pattern is that of a single particle the total yield depends only weakly on the beam energy $\gamma$. When the spot size is large the total COTR yield falls off rapidly. For intermediate values of $k \sigma_{r}$ we must rely on numerical integration to obtain a yield number. Figure 6.2 shows a yield calculation as a function of spot size. Shown in the plot are the asymptotic solutions for very small and very large spot sizes.

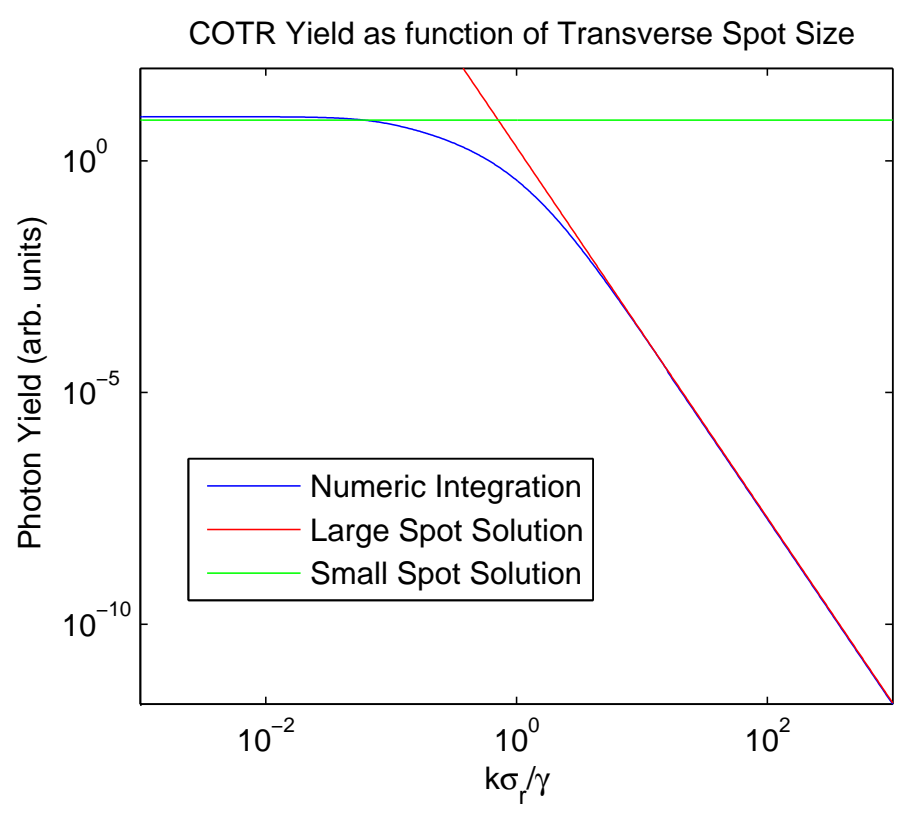

Figure 6.2: Total COTR yield as a function of beam transverse spot size (blue). Also shown are the asymptotic limits for small (green) and large (red) spot sizes.

In the experiment described in this chapter the spot size at the radiator was typically $\sim 50 \mu \mathrm{m}$ rms with $\sim 1 \mathrm{pC}$ of charge. Evaluating the numerical integral of equation 6.7 gives expected yields of $76 \mathrm{fJ}$ of COTR photon energy at the fundamental and $5 \mathrm{fJ}$ at the second harmonic. Of course, actual experimental values could vary 
greatly from this with changes in charge or spot size or shape.

To study the microbunching, the most important dependencies of the COTR yield are those that enter through the bunching factors, $b_{n}$. Less interesting is the total yield which can vary greatly with small changes in the charge or transverse spot size, both of which are known to change with small linac tuning changes or day-to-day variation in the UV laser to the gun. Furthermore, as can be noted from equation 6.7 , the total amplitude of each harmonic varies differently as a function of spot size for each harmonic $n$. This makes comparing amplitudes between different harmonic difficult without precise knowledge of the transverse beam profile. Therefore, for comparison to the experiment, we rely solely on the relative shape of each harmonic as a function of the bunching factor variables: $R_{56}$ and $\eta$. We write a simpler equation stressing this dependence:

$$
I_{C O T R, n} \propto\left[J_{n}\left(n k_{L} R_{56} \frac{\eta}{\gamma_{0}}\right)\right]^{2} \exp \left[-\left(n k_{l} R_{56} \frac{\sigma_{\gamma}}{\gamma_{0}}\right)^{2}\right]
$$

\subsection{Experiment Layout and Methodology}

The transport of the electron beam into the experimental chamber has been described in chapter 5. The laser is brought to the experiment optical table containing the main experiment chamber, diagnostic optics, and optics for the laser. Here, a quadrant detector and a pair of diodes monitor the laser. In early attempts at the experiment, the laser was sent into the chamber via a 8" cube immediately in front of the chamber. The 8" cube housed a dielectric coated pellicle mirror with a thickness of $\sim 10 \mu \mathrm{m}$. The electron beam passed through the mirror while the laser reflected through $45^{\circ}$ toward the undulator. However, it was soon found that the mirror caused excessive scatter to the electron beam. Later calculations based on [61] showed that the mirror increased the transverse emittance to $\sim 20 \mathrm{~mm}$-mrad compared to an initial emittance of $2 \mathrm{~mm}$-mrad. The experiment was therefore modified to send the laser to a viewport at the second dogleg bend (see chapter 5, figure 5.3, 'BEND 4400'). A smaller optical table was installed at this location for focusing and steering optics, as well as an alignment laser used during experiment installation. 


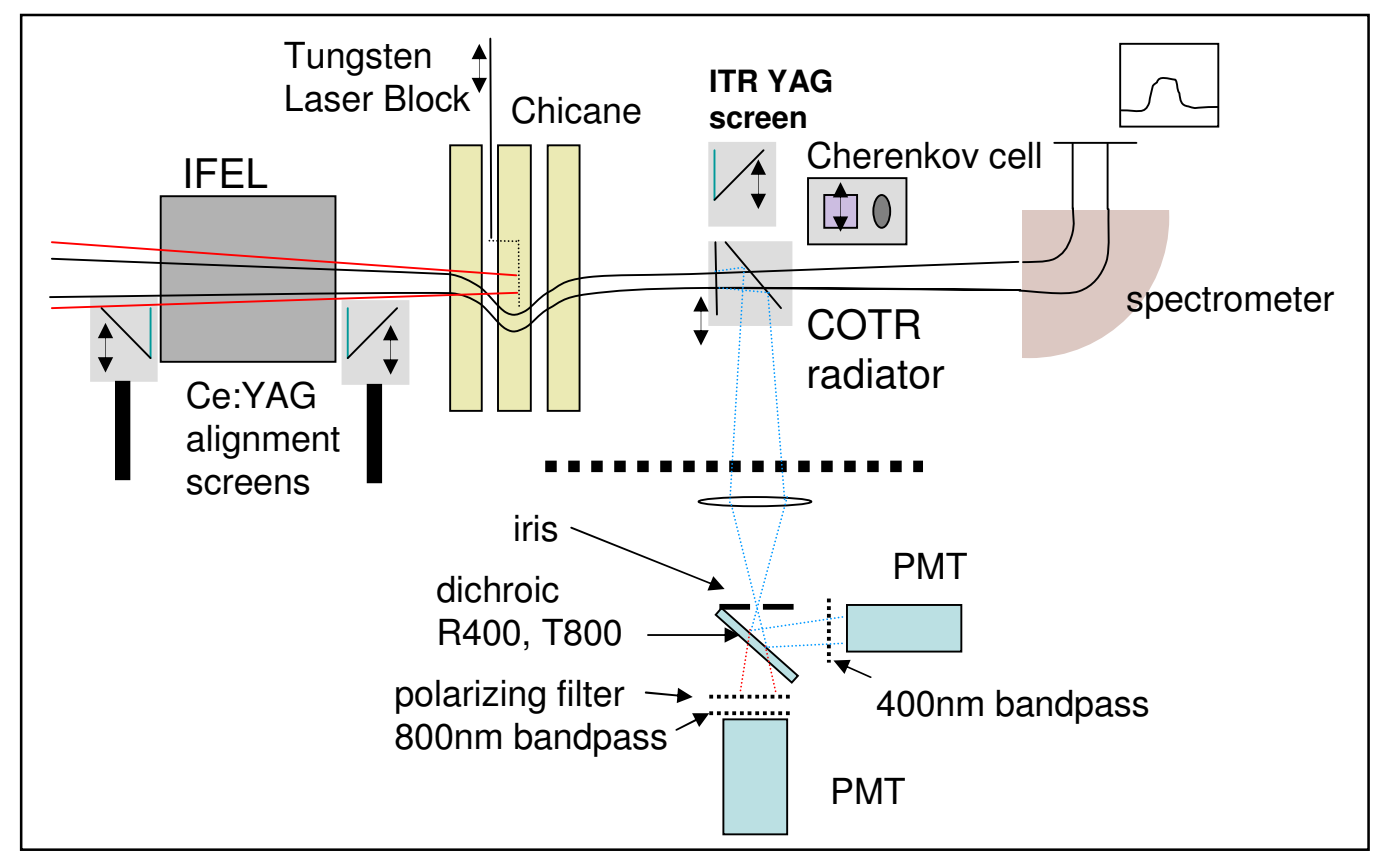

Figure 6.3: A schematic layout of the microbunching experiment.

The layout for the experiment is shown in figure 6.3. Both beams are focused going into the experimental chamber housing the undulator, chicane, and the COTR radiator as well as several diagnostic screens mentioned in §5.3.1. Two of these screens, called 'IFELYGUP' and IFELYGDN', are attached fore and aft of the undulator for overlapping the laser and electron beam while a third screen (called 'ITRYAG') is used to minimize the electron beam spot size at the radiator. The Cherenkov radiator sits just behind the COTR radiator and ITRYAG screen. After the COTR radiator the beam exits the experimental chamber to the energy spectrometer.

The chamber, spectrometer screen, and spectrometer dipole are arranged such that the spectrometer images the electron beam at the ITRYAG screen and COTR radiator onto the spectrometer screen. The electron beam is focused at the plane of the radiator and ITRYAG screen in order to maximize COTR output. This also gives maximum resolution at the energy spectrometer. 
To avoid damaging the COTR radiator and to reduce background due to the laser, a tungsten beam stop is inserted in the middle of the chicane to intercept the laser after it is used for the IFEL. The radiator consists of two pellicle mirrors, one normal to the beam and a second at $45^{\circ}$ to send the light to a pair of photomultiplier tubes (PMTs). As one would expect, the COTR pellicles introduce additional scatter to the beam. However, since the pellicles are located at the object plane of the spectrometer, the horizontal scatter is refocused and does not contribute to apparent energy broadening at the spectrometer YAG screen. In the vertical dimension, however, the spectrometer does not refocus and the image at the spectrometer becomes a vertical stripe when the COTR radiator is inserted.

The light from the COTR pellicles is sent up toward a standard F-mount camera lens that images the back of the first pellicle onto an iris just in front of the PMTs. This allows the PMT to collect the maximum amount of COTR light and also helps in the initial alignment. The iris is closed down to select only the pellicle from the lens's field of view. This helps reduce background at the fundamental from laser light scattered inside the vacuum chamber. Just after the iris, the COTR light is split using a dichroic mirror to reflect the second harmonic to one PMT and transmit the fundamental to the second. Each PMT also has a bandpass filter attached to select only the given harmonic and also help protect against ambient light. Finally, a polarizing filter is placed in front of the 800nm PMT to further reduce background due to the laser.

Table (6.1) gives a list of experimental parameters including beam, laser, and magnet values. The beam values for the initial energy spread and temporal pulse length were obtained from the data itself. Data are taken in runs of 500-4000 beam shots at the $10 \mathrm{~Hz}$ repetition rate of the accelerator. Just as with the IFEL harmonics experiment (chapter 4), data are taken as a cross-correlation between the laser and electron beam. A random delay is set using the fast delay stage mentioned in $§ 5.1$. For each event an image of the beam at the energy spectrometer is acquired along with numerous diagnostics including the COTR PMTs and diodes for the laser. From the energy profiles a beam energy spread is extracted and plotted against the delay forming a cross-correlation scatter plot. When the electron beam and laser are in time 


\begin{tabular}{|l|c|}
\hline Parameter & Value \\
\hline Electron Energy & $60 \mathrm{MeV}$ \\
Energy Spread & $30 \mathrm{keV}$ (typ.) \\
Electron Pulse length & $0.8 \mathrm{ps} *$ (typ.) \\
Electron Spot Size & $100 \mu \mathrm{m}$ (nom.) \\
Bunch Charge & $1 \mathrm{pC}$ (nom) \\
Laser Wavelength & $785 \mathrm{~nm}$ \\
Laser Energy & $0.65 \mathrm{~mJ} / \mathrm{pls}$ \\
Laser Pulse length & $0.8 \mathrm{ps}(\mathrm{nom}$.) \\
Laser Spot Size & $200 \mu \mathrm{m}$ \\
Undulator Period & $1.8 \mathrm{~cm}$ \\
Number of Periods & 3 \\
Undulator Strength $\left(a_{w}\right)$ & 0.46 \\
Chicane $R_{56}$ & $0.04-0.16 \mathrm{~mm}$ \\
\hline
\end{tabular}

Table 6.1: Experimental Parameters for attosecond bunch train production. All widths are given as fwhm. ${ }^{*}$ Estimated from the data.

the IFEL modulates the electron beam energy and the COTR signal is strongest.

The cross-correlation is an important technique for this experiment, especially for the COTR signal. The fact that the signals from the PMTs are strongest when the laser and electron beam are in-time excludes other processes as background including harmonic generation from the laser hitting surfaces in the chamber, incoherent transition radiation, bremsstrahlung, or undulator radiation, all of which would occur regardless of relative timing between the two beams.

Figure (6.4) depicts an example data set showing the cross-correlation scatter plots of the energy spread as well as the fundamental and second harmonic COTR signals. Notice that when the delay is set for laser-electron temporal overlap, all events show interaction. This indicates that the timing jitter is less than the electron and laser pulse widths. The temporal width of the slopes on the cross-correlation give an upper bound on the jitter of 0.2 ps. In this particular electron beam configuration there is a smaller electron pulse behind the main pulse. The COTR signals show the same characteristic structure to the electron beam. The fundamental COTR signal has a large offset between laser-on and laser-off events indicating there is still significant bleed-through of laser light to the detector. This bleed-through also contributes 

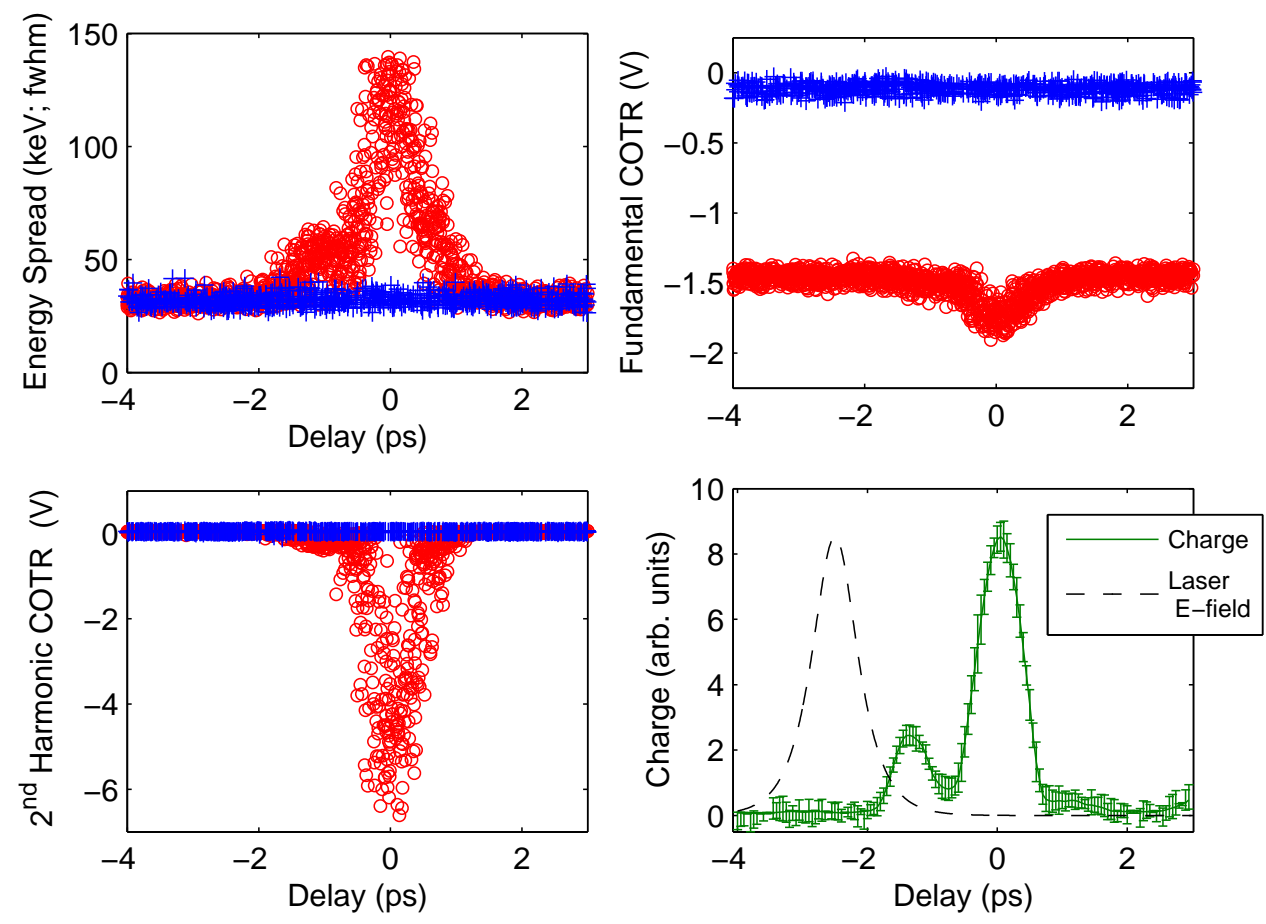

Figure 6.4: Example data run of the IFEL microbunching experiment containing 2130 events. Top left is the electron energy spread as a function of delay with laseron events in red (circles), laser-off in blue (crosses). Also shown are the COTR raw signals at the fundamental (top right) and second harmonic (bottom left). The bottom right figure shows the current profile calculated by deconvolving the laser pulse from the cross-correlation data.

significantly to the noise on the signal.

Deconvolution of the laser temporal profile from the electron energy modulation (fig. (6.4) bottom right) estimates the main electron pulse temporal length of 0.8 ps fwhm. The laser temporal profile, obtained from autocorrelation, is 0.55ps. The IFEL modulation is given by the difference in quadrature of the total energy spread and the initial energy spread $\left(\sigma_{I F E L}=\sqrt{\sigma_{T O T}^{2}-\sigma_{\text {init }}^{2}}\right)$. The energy modulation crosscorrelation is binned as a function of delay and deconvolved from the laser field profile using a genetic algorithm. The deconvolution is performed several times, re-seeding with an energy modulation spectrum that is varied using the variances of the binned data. 


\subsection{Results from the Experiment}

To explore further the dependence of COTR output on the IFEL modulation we plot the COTR signals versus the IFEL modulation strength. This is shown in figure (6.5). Here, the raw COTR signals have been inverted, rescaled, and the baseline signal (with no IFEL interaction) subtracted. The IFEL modulation is given by the difference in quadrature of the total energy spread and the initial energy spread $\left(\sigma_{I F E L}=\sqrt{\sigma_{T O T}^{2}-\sigma_{\text {init }}^{2}}\right)$. The only cut applied to the data here is on the delay timing to select events near the interaction overlap.
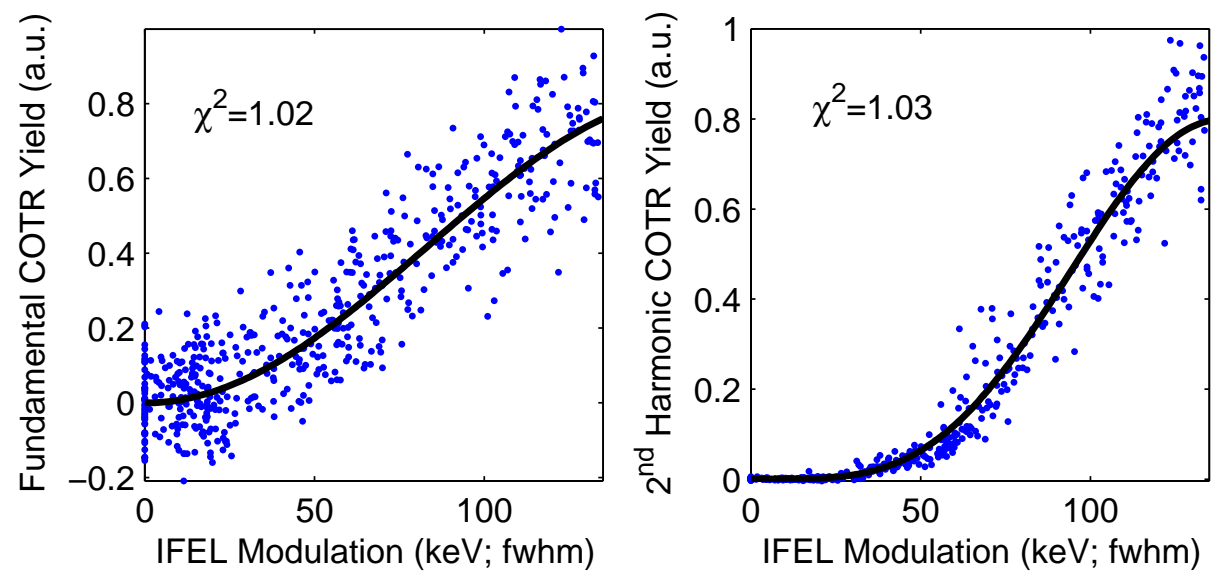

Figure 6.5: Scatter plot of COTR signals versus IFEL modulation for a single run. Each point is a single interaction of the laser and electron beam. The amplitude of the IFEL interaction varies due to the delay scan. The solid lines give the analytic form (eqn. (6.10)).

Shown with the data for each harmonic is the analytic form (eqn (6.10)) using the known value of $R_{56}=0.2 \mathrm{~mm}$ and taking $\eta=0.5 * M / E_{0} * 1.3$ where $M$ is the measured IFEL modulation fwhm and $E_{0}=60 \mathrm{MeV}$ is the beam energy. For a constant modulation across the entire beam, we would have $\eta=0.5 * M / E_{0}$ (recall $\eta$ is the amplitude of a sinusoidal modulation). However, since the measured $M$ is an average across the entire beam including electrons not modulated (either out-oftime or off-axis transversely), we expect the radiating portion of the beam to have a larger modulation $\eta$. Allowing $\eta$ as a fit parameter and minimizing $\chi^{2}$ we obtain 
$\eta=0.65 * M / E_{0}$. This is also consistent with an extended analytic theory taking into account laser and electron beam form factors. The fit of the analytic form to the data shows very good agreement with $\chi^{2}=1.02$ for the fundamental data and $\chi^{2}=1.03$ for the second harmonic. For this fit the variance for each data point is calculated from the deviation of the nearest neighbors, binned in steps of $10 \mathrm{keV}$ in the IFEL modulation.

With the COTR signals plotted in this way the difference in the two harmonics is clear. As the total IFEL modulation decreases the amount of second harmonic COTR falls off more rapidly than the fundamental. This is due both to the differing Bessel function dependence as well as to the greater sensitivity to energy-spread-based washout from the exponential term in equation (6.10).

In addition to the modulation strength, $\eta$, the COTR output varies strongly with the chicane $R_{56}$. The chicane design includes coils to give adjustment of the magnetic field by up to $\pm 20 \%$ in order to optimize microbunch formation. To study the dependence of COTR output versus chicane strength, a number of short data runs were taken with the chicane strength varied between each run. A fit was done to each cross-correlation, an amplitude extracted and an error deduced using Bootstrap method[62]. A longer description of the Bootstrap method used is given in appendix B. This involves using the data itself to infer a confidence interval by taking subsets of the data set and repeating the analysis, in this case a Gaussian fit through the correlation plot. The deviation in the resulting collection of fit parameters gives the confidence interval. The results are shown in figure (6.6).

The main difference between the two curves in the chicane scan comes at low values of $R_{56}$ where the second harmonic again rolls off faster than the fundamental. At higher values both signals show a roll-off indicating that optimum bunching is being reached for $R_{56}=0.13-0.16 \mathrm{~mm}$. The inset shows the analytic form over a larger range of $R_{56}$ taking $\eta=0.65 * M / E_{0}$ as before where for these runs the mean interaction $M=100 \mathrm{keV}$ fwhm. These runs were taken over a period of $\sim 1$ hour during which both the mean interaction strength $M$ and the total charge drifted (see figure 6.7). As a result, the fit to the analytic form is relatively poor compared to the COTR versus modulation data shown in figure (6.5). Nevertheless, the data agrees 


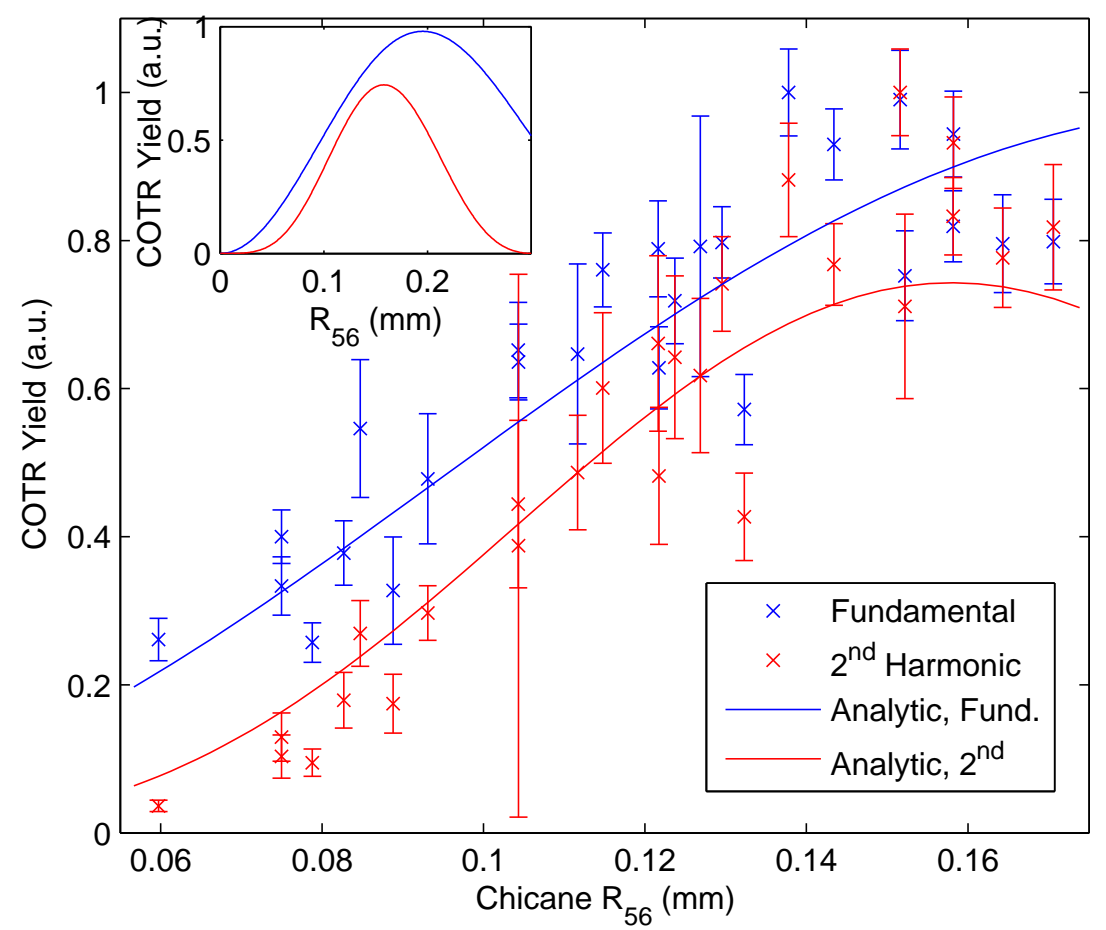

Figure 6.6: A scan of the chicane $R_{56}$. The first and second harmonics are shown together with the analytic form overlaid. Each data point represents a run of 500 events, fitted with errors found by the Bootstrap technique. The inset shows the analytic function for the COTR output (eqn. (6.10)).

well qualitatively with the analytic form. The analytic solutions reach a maximum at $R_{56}=0.22 \mathrm{~mm}$ for the fundamental and $0.18 \mathrm{~mm}$ for the second harmonic. The actual COTR signals peak for lower $R_{56}$ due to the mean being pulled down by more strongly interacting events that are overbunched. Figure 6.4 shows events with modulation up to $140 \mathrm{keV}$. For these events, optimal bunching would occur at $R_{56}=0.12 \mathrm{~mm}$. This demonstrates the importance of independently measuring the bunching through the COTR to optimize the $R_{56}$.

A number often quoted as a figure of merit for bunching is the bunch parameter $b_{n}$, equal to the Fourier coefficient for longitudinal charge density. In our case: $b_{n}=$ $J_{n}\left(n R_{56} k_{L} \eta\right) \exp \left[-\frac{1}{2}\left(n \sigma_{\gamma} / \eta\right)^{2}\right]$ as defined earlier. For the maximum $R_{56}=0.16 \mathrm{~mm}$, an initial energy spread $\sigma_{\gamma}=3.3 \mathrm{e}-4$ (corresponding to $33 \mathrm{keV}$ fwhm), and the modulation 

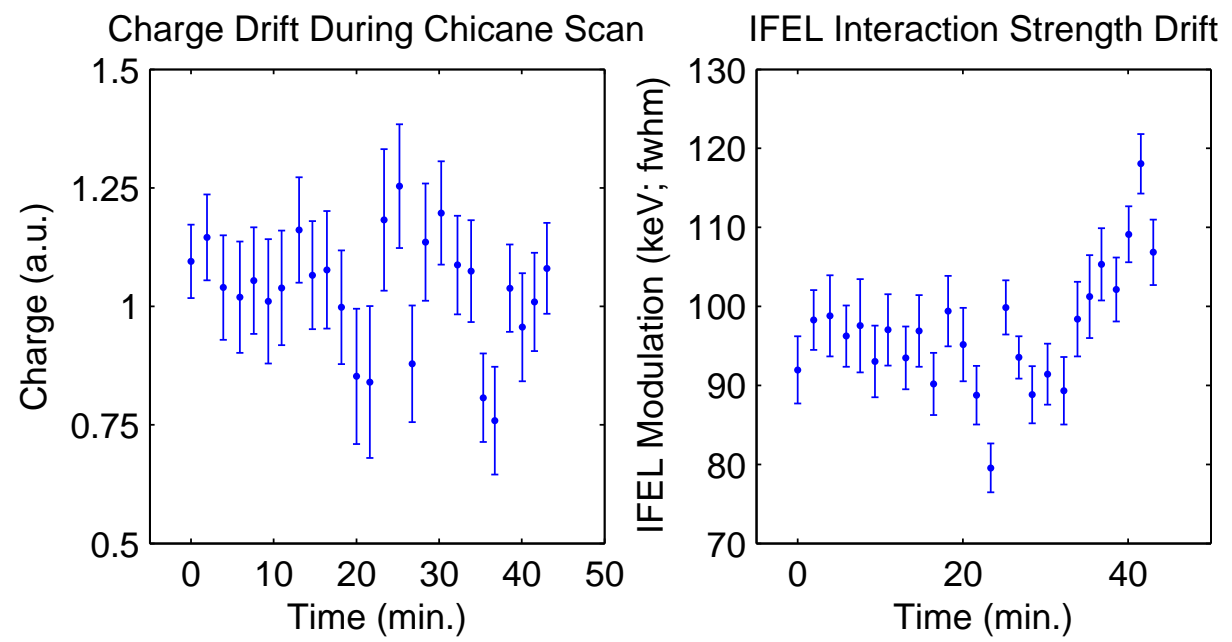

Figure 6.7: Drifts during the chicane scan. Both the charge (left) and IFEL modulation strength (right) drifted significantly while taking the chicane scan data leading to a larger spread in the data.

$M=100 \mathrm{keV}(\eta=1.1 e-3)$ we obtain $b_{1}=.52$ and $b_{2}=0.39$. At this level of bunching the individual microbunches have a temporal pulse width of $\sim$ 410as.

This experiment has succeeded in producing stable, optically spaced attosecond bunch trains. With the laser to electron beam delay held constant, a microbunched beam is produced on every shot with a jitter corresponding to $\leq 20 \%$ on the bunching factor. This stability will be important in future experiments looking to measure net acceleration of optical microbunches. The first such experiment uses the ITR accelerator (\$1.3.1) as the second stage and is presented in the next chapter. Future experiments will also explore using these microbunches in optical scale accelerators where the net energy gain will significantly separate the microbunches from the unbunched portion of the beam. Prior to laser powered acceleration experiments, the microbunched beam will also be used to probe candidate accelerator structures by studying the wakes produced by the passage of a microbunched beam through a near-field optical structure[63]. This research effort might one day lead to novel devices producing energetic beams at very high repetition rates. With the application of lithographic processing techniques and commercially mass produced laser components these devices could be made cheap, compact, and reliable. 


\section{Chapter 7}

\section{Net Acceleration of Electrons with Light}

With the success of the microbunching experiment in producing a reliable, microbunched electron beam the natural next step is to accelerate this beam in a second stage. Obtaining direct net acceleration from a near infrared laser would mark a significant milestone in the research program. By combining the microbunching hardware with a second stage accelerator, additional challenges are introduced. Most notably, the phase of the microbunches relative to the second stage accelerating field must be well controlled or at least measured with sufficient accuracy to make meaningful correlations between the acceleration phase and the resulting electron energy spectra. Ultimately, to be useful as an accelerator, the phase will need to be controlled to probably within less than a degree of an optical cycle to minimize energy jitter and spread. However, for initial experiments exploring the feasibility of laser acceleration, we can accept less stringent control of the phase.

In addition to the phase control, the additional hardware of a net acceleration experiment makes for an ever-increasing challenge. Several different systems must work simultaneously to achieve the goal of net acceleration. The experiment requires additional laser focusing and steering for the second stage, and a system to scan the laser timing. Successful net acceleration at optical frequencies is therefore a considerable technical achievement. With the lessons learned from this first effort, the 
techniques, methods, and hardware development can be applied to future experiments in direct laser acceleration research.

\subsection{The Second Stage of the Net Acceleration Ex- periment}

The ITR accelerator structure (§1.3.1) will serve as the second stage for the net acceleration experiment. This mechanism is capable of producing $\sim 50-70 \mathrm{keV}$ fwhm laser-electron interactions with $0.5 \mathrm{~mJ}, 1$ ps laser pulses focused to a $\sim 100 \mu \mathrm{m}$ spot size. The ITR accelerator isn't likely to be useful as an accelerator long-term owing to the scatter of the electron beam passing through the foil and the very poor energy efficiency. However, for an initial experiment it has several advantages. Most notably, the mechanism has been used and debugged in prior experiments[28]. Thus, electronics and software for controlling the mechanism already existed well prior to the net acceleration experiment and did not need to be designed. Also, like the IFEL process, the ITR acceleration process can be done with electron and laser spots much larger than the laser wavelength, greatly simplifying the focusing and steering of both beams.

Unfortunately, besides the long-term ineligibility of the ITR mechanism, the use of the ITR setup for this experiment has other disadvantages. The small interaction makes it difficult to observe the net energy shift from background noise. The total energy shift of the microbunched beam is given by the integral of the longitudinal bunch density (equation 2.31) times the sinusoidal energy shift imparted at the second stage.

$$
\begin{aligned}
& \Delta E=\frac{1}{\lambda_{l} \rho_{0}} \int_{0}^{\lambda_{l}} \rho_{0}\left[1+2 \sum_{n=1}^{\infty} b_{n} \cos \left(n k_{l} z\right)\right] A \cos \left(k_{l} z+\phi\right) d z \\
& \Delta E=\frac{2 A b_{1}}{\lambda_{l}} \int_{0}^{\lambda_{l}} \cos \left(k_{l} z\right) \cos \left(k_{l} z+\phi\right) d z \\
& \Delta E=A b_{1} \cos \phi
\end{aligned}
$$


Here, $\lambda_{l}=2 \pi / k_{l}$ is the laser wavelength, $b_{n}$ are the bunch coefficients, $A$ is the amplitude of the ITR interaction, and $\phi$ is the phase difference between the IFEL and ITR drive lasers. We notice first that for the total net acceleration only the first harmonic of the bunching matters. Of course, to minimize the energy spread of an accelerated bunch, the microbunches must also be narrow in time necessitating stronger higher harmonics to the bunch density. Since $A$ is the amplitude of the sinusoidal modulation, we have $A \sim 0.5 \times \sigma_{E, \text { fwhm }}$. The maximum value of $b_{1}$ is $\max \left(J_{1}\right)=0.6$. Therefore the strongest net acceleration signal we can hope to see from a $50 \mathrm{keV}$ fwhm ITR interaction is $25 \times 0.6=15 \mathrm{keV}$ energy shift. Several other factors including phase jitter and wash-out of the microbunching due to the initial energy spread shrink this further. The small interaction signal therefore makes this a very challenging experiment.

Another issue with using the ITR setup as the accelerator comes from the $1 / \gamma$ interaction angle. Because the laser crosses the beam at an angle, for a given longitudinal slice within the beam, electrons at different transverse positions see a different phase of the laser field (see figure 7.1).

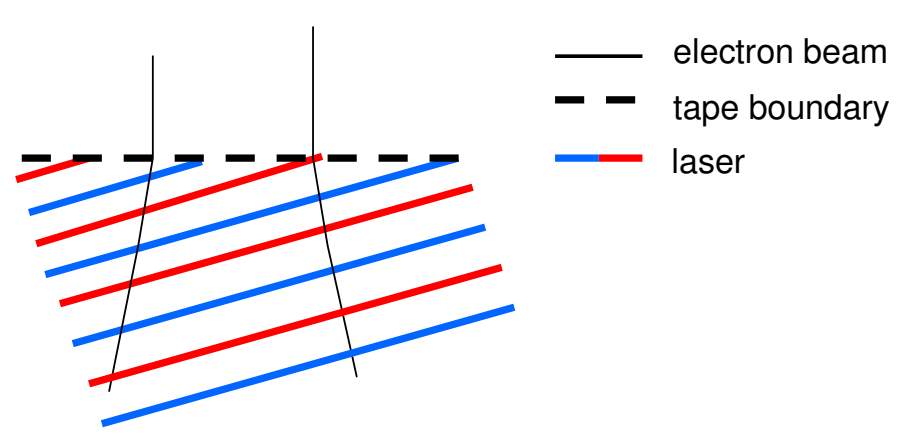

Figure 7.1: An illustration of the ITR-microbunch horizontal phase problem. Due to the laser coming in at an angle relative to the electron beam, portions of a single microbunch will witness different phases of acceleration in the ITR interaction.

The transverse phase oscillates at the wavelength times the angle: $\lambda_{l}(1 / \gamma)=80 \mu \mathrm{m}$. 
To observe acceleration, we must limit the electrons to one half cycle, or $40 \mu \mathrm{m}$. The measured spot size at the focal point in the experimental chamber is $\sim 150 \mu \mathrm{m} \mathrm{fwhm}$. We would therefore be unable to observe any net acceleration without limiting the beam. To do this, a narrow vertical slit is placed just after the ITR accelerator to select a horizontally narrow portion of the beam.

\subsection{Expected Signals and Monte Carlo Simulations}

Although the main interest in the experiment is observing the shift of the total energy of the beam, the net acceleration also affects the total energy spread and asymmetry of the beam energy distribution. Figure 7.2 shows the progression of the electron phase space and energy spectrum for a single cycle of optical phase. An inset gives the parameters used for this plot. As can be seen from the energy spectra, the spectral asymmetry changes as a function of phase along with the mean energy. For this experiment, due to the relatively small ITR interaction, the energy shift is much smaller than the energy spread induced by the IFEL and ITR interactions. This makes identifying a 'captured fraction' from the energy spectrum very difficult.

In the prior experiments (ch 4 and ch 6), a particle tracking code (§2.5) was used to simulate the experiment before hand and obtain estimates of the interaction strength. However, to simulate many different laser-electron interactions, each requiring thousands of particles to build up a simulated energy spectra, would require excessive computing power. Therefore, in order to simulate a data run of several hundred laser-electron interactions, a different method is used employing a Monte Carlo code. This code does not explicitly track particles through the magnet and laser fields. Instead, the code divides a beam with a starting energy spectrum into several sub-spectra with separate charge, laser phase, and laser amplitude. Each energy spectra is modified analytically with the interaction amplitude estimated from

previous particle tracking simulations. The energy spectra are then added back up to form the energy spectrum for a single event. For each energy spectrum, the IFEL to ITR laser phase can be changed, along with other jitter parameters such as transverse position, centroid energy, etc. 

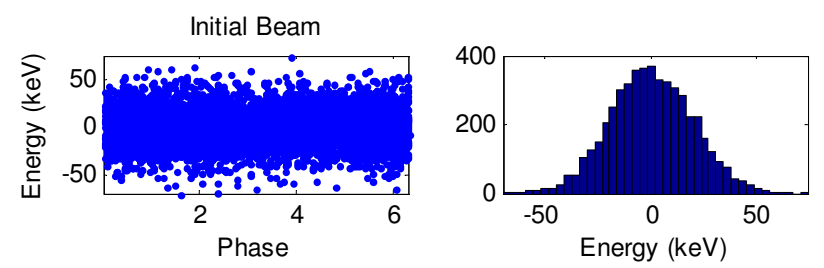

$$
\begin{aligned}
& \sigma_{E}=30 \mathrm{keV} \\
& \mathrm{M}_{\text {IFEL }}=70 \mathrm{keV} \\
& \mathrm{M}_{\text {ITR }}=50 \mathrm{keV} \\
& \Delta \mathrm{E}_{\text {acc/dec }}= \pm 7.5 \mathrm{keV}
\end{aligned}
$$
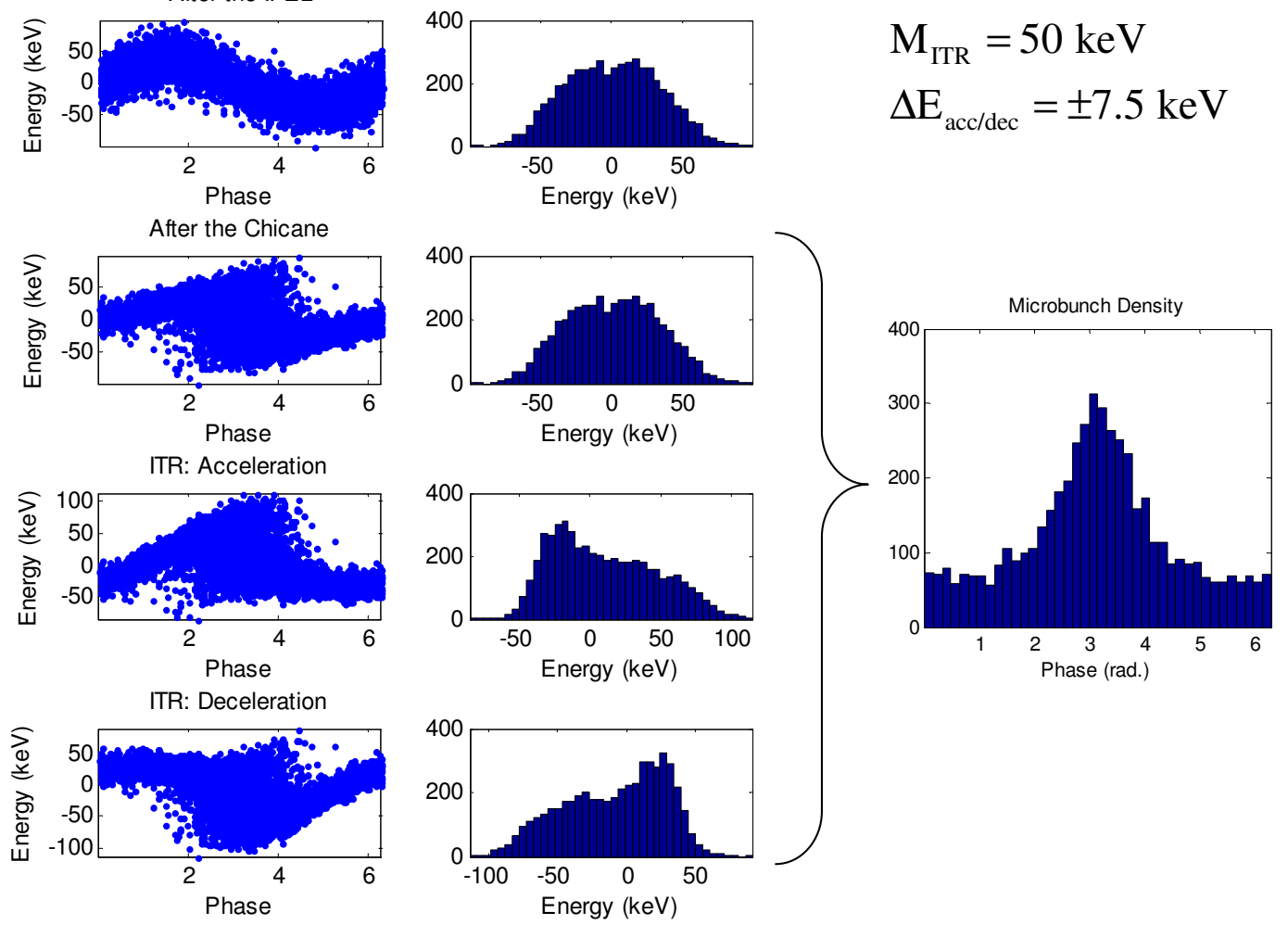

Figure 7.2: Evolution of a microbunch in the net acceleration experiment. Shown are the longitudinal phase space (left column) and energy spectrum (right column) starting before the IFEL and finishing with accelerating and decelerating cases for the ITR interaction. Notice that in the bottom two plots the peaked portion of the energy spectrum actually corresponds to an unbunched portion of the beam in the phase space plot. A separate figure to the right shows the longitudinal density after the chicane. 
Figure 7.3 shows a Monte Carlo simulation of the experiment. In this case, the phase is adjusted as a saw-tooth function in time such that the acceleration signal varies sinusoidally in time. The bottom right plot shows an fft of the various signals showing clear content at the phase drive frequency of $0.1 \mathrm{~Hz}$. The other three plots are the phase-signal correlation plots. Notice that the energy centroid shift and asymmetry are $\pi$ out of phase with respect to one another, while the energy spread oscillation is $\pi / 2$ out of phase. The asymmetry is defined as the lower-width half-maximum minus the upper-width half-maximum, divided by the full-width half-maximum.
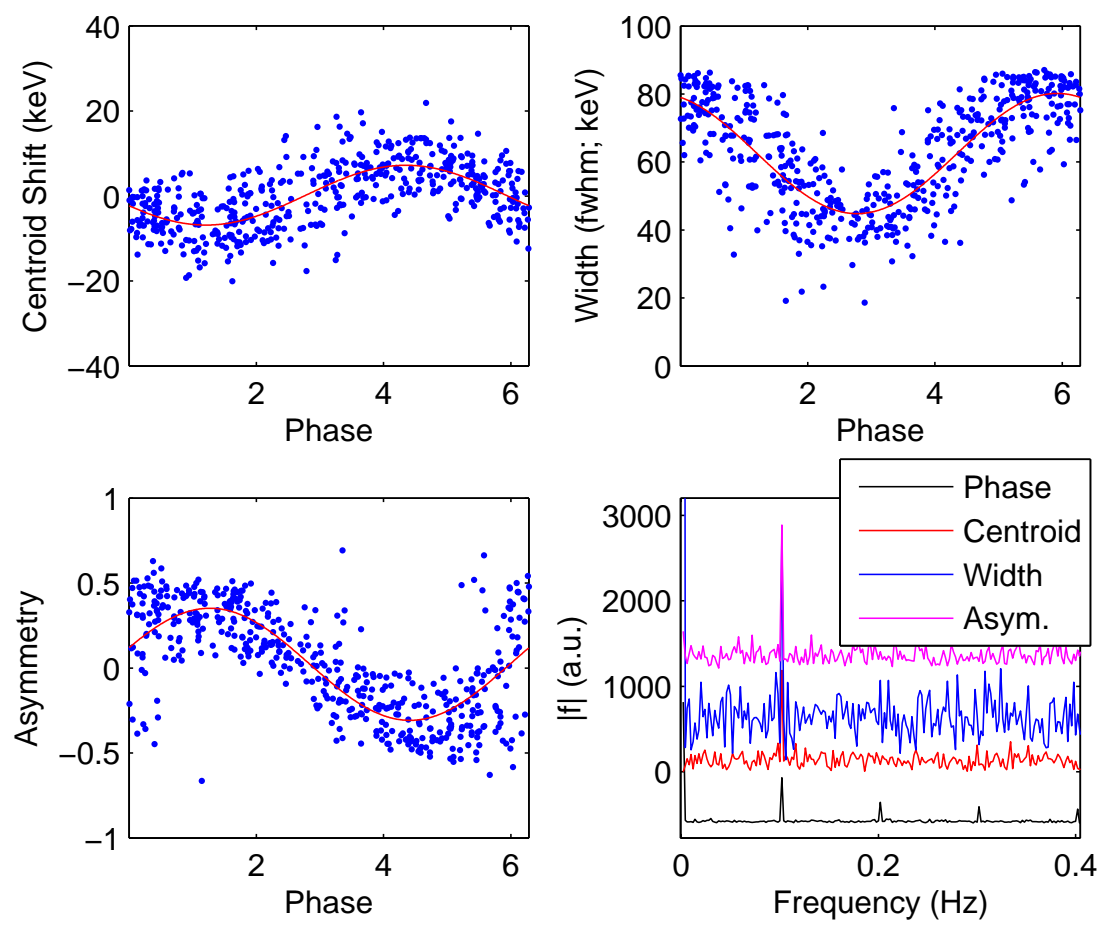

Figure 7.3: A Monte Carlo simulation of the net acceleration experiment with 500 laser-electron interactions.

The Monte Carlo includes reasonable estimates for the jitter of $25^{\circ}$ of phase, 5 $\mathrm{keV}$ on the energy centroid and energy spread jitter, and $30 \mu \mathrm{m}$ of position jitter. The Monte Carlo simulation indicates that even with these sources of jitter, a clear signal should be measurable. The saw-tooth scan of the phase should also be helpful in finding signals from the noise. 


\subsection{Experiment Layout}

The net acceleration experiment builds on the hardware for the microbunching experiment (§6.2). In addition to the hardware and software of the ITR stage[25], the experiment adds a new optical path to drive the ITR process. Figure (7.4) shows the layout for the net acceleration experiment.

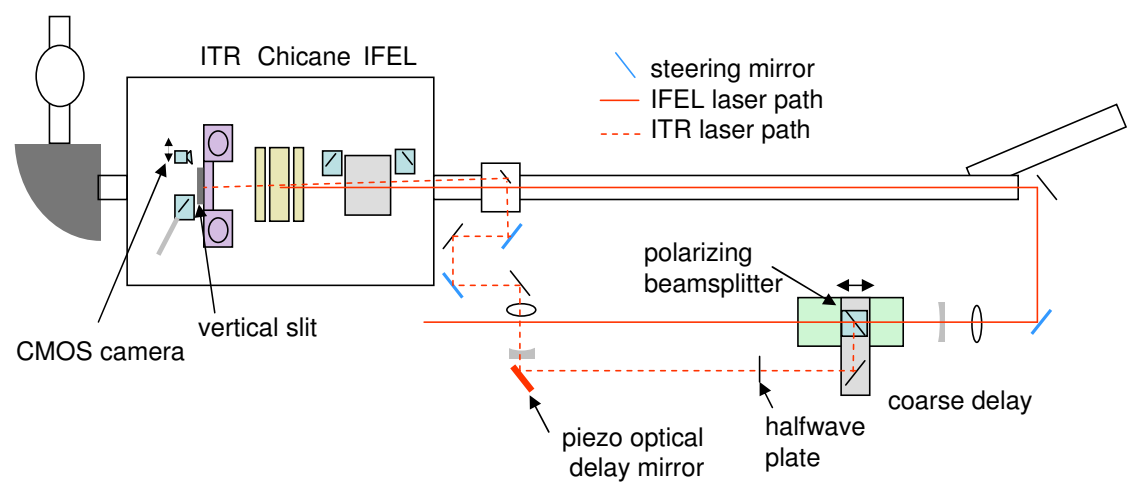

Figure 7.4: Layout of the net acceleration experiment. The laser is split at a polarizing beamsplitting cube (PBS) to drive both the ITR acceleration and the IFEL. The ITR path includes a piezo driven mirror for varying the optical phase. Each arm is $\sim 5 \mathrm{~m}$ in path length. Interferometric noise measurements indicate the optical paths are stable to within $\sim 20 \mathrm{~nm}$ or 2 parts per billion of the total path.

The laser is split in order to power the additional second stage. This is done using a polarization beamsplitting cube near the experiment chamber and a half-waveplate (HWP) (located in the laser room) that rotates the initially horizontally polarized laser out of the regen. This waveplate is remote controllable to allow adjustment of the splitting ratio during the experiment. The beamsplitter is positioned such that the horizontal component of the laser light is transmitted to the IFEL while the vertical component is reflected and sent to the ITR optical path. In the ITR arm, a second waveplate rotates the polarization back to horizontal as needed to drive the ITR process. The ITR path includes additional telescoping and steering mirrors 
needed to get the appropriate spot size and positioning in the experiment. The ITR laser enters the vacuum through a 4.5" cube just upstream of the main experimental chamber. Inside the cube is a 1" dielectric mirror positioned off-axis with respect to the nominal electron beam trajectory. This can be done since the ITR process requires the laser at an angle of $1 / \gamma=0.8 \mathrm{mrad}$ with respect to the electron beam. The 4.5" cube sits $\sim 1 \mathrm{~m}$ upstream of the ITR assembly, thus the laser spot is centered $8 \mathrm{~mm}$ apart from the electron beam allowing the mirror to reflect the laser out while avoiding the electron beam.

The beamsplitter and the first mirror in the ITR path rest on a common stage that can be moved to allow a coarse timing adjustment of the IFEL and ITR optical paths. This is used to achieve both ITR and IFEL interactions at the same timing delay of the fast delay mirror in the laser room ( 55.1$)$. Prior to the experiment, the coarse timing overlap can largely be achieved by searching for an interference pattern when the two laser spots are overlapped in the experimental chamber. However, any steering of the laser during the experiment can introduce few picosecond timing shifts. Also, the chicane causes a delay of the electron beam of $\sim 1 \mathrm{ps}$, that varies depending on the chicane strength. Because of these issues, a remote controllable, few ps range delay is needed between the IFEL and ITR lasers.

In addition to the long travel delay used for picosecond scale adjustment of the ITR-IFEL delay, the optical path of the ITR laser also includes an adjustment for the optical phase of the ITR to IFEL delay. This phase adjustment is done using a mirror mounted on a piezo driven mount. The three piezos are driven by a single source, producing a longitudinal shift of the mirror of up to 8 microns. This changes the ITR laser path by a small amount. With this mirror, the phase of the ITR relative to the IFEL laser can be varied in a controlled, repeatable manor. Moving the mirror also shifts the ITR laser spot transversely, however this shift is much less than the transverse spot of the laser and gets further demagnified by the downstream lenses used to focus the laser onto the tape.

The distance between the beamsplitter and the interaction point in the chamber for each laser path is $\sim 5 \mathrm{~m}$. The two path lengths were set equal using the slow delay stage and looking for fringes to appear in a CCD image of the overlapped, 
1 ps long, laser spots. The experiment effectively constitutes a large interferometer, where the electron beam takes the place of the IFEL laser before recombining with the ITR laser. A key piece of information in performing the experiment is knowing the relative phase between the electron microbunches and the ITR laser in the second stage ITR accelerator. Unfortunately, there is no easy way to obtain this information directly from the electron beam and lasers inside the experimental chamber. Potential electron radiation schemes such as COTR are too weak for interferometry based phase information. Meanwhile, the ITR and IFEL lasers are too strongly focused to allow pick-off mirrors inside the chamber. Such mirrors would quickly damage. They would also likely disturb the electron beam.

In order to obtain the phase between the IFEL and ITR paths a second optical path that closely follows the $800 \mathrm{~nm}$ laser for the experiment is installed in the experimental tables. Figure 7.5 shows the layout of the phase monitor. The additional optics are setup as a Michelson interferometer with one path entering the vacuum at the dogleg port along with the portion of the $800 \mathrm{~nm}$ used to drive the IFEL. The second arm of the phase monitor interferometer is arranged such that the piezo driven delay mirror for the ITR is also sampled. Just upstream of the experimental chamber, inside the 4.5" cube, a mirror located below the nominal beam height is used to bring the phase monitor laser back out of the vacuum where it is then recombined with the other arm of the interferometer. The lasers are overlapped on a CCD (charged coupled device) and the resulting fringe pattern in used to interpret the phase of the laser in the interferometer. The phase monitor is driven by a $T E M_{00} 1064 \mathrm{~nm}$ NPRO laser[64].

Images from the phase monitor CCD are acquired by a frame grabber. A regionof-interest (ROI) is defined to select a portion of the fringe pattern. The image is summed and the resulting lineout is Fourier transformed. The phase of the fringes is obtained from the phase of the fft element corresponding to the fringe modulation in the image. This phase information is transferred to the main data acquisition computer for writing to the data files. 


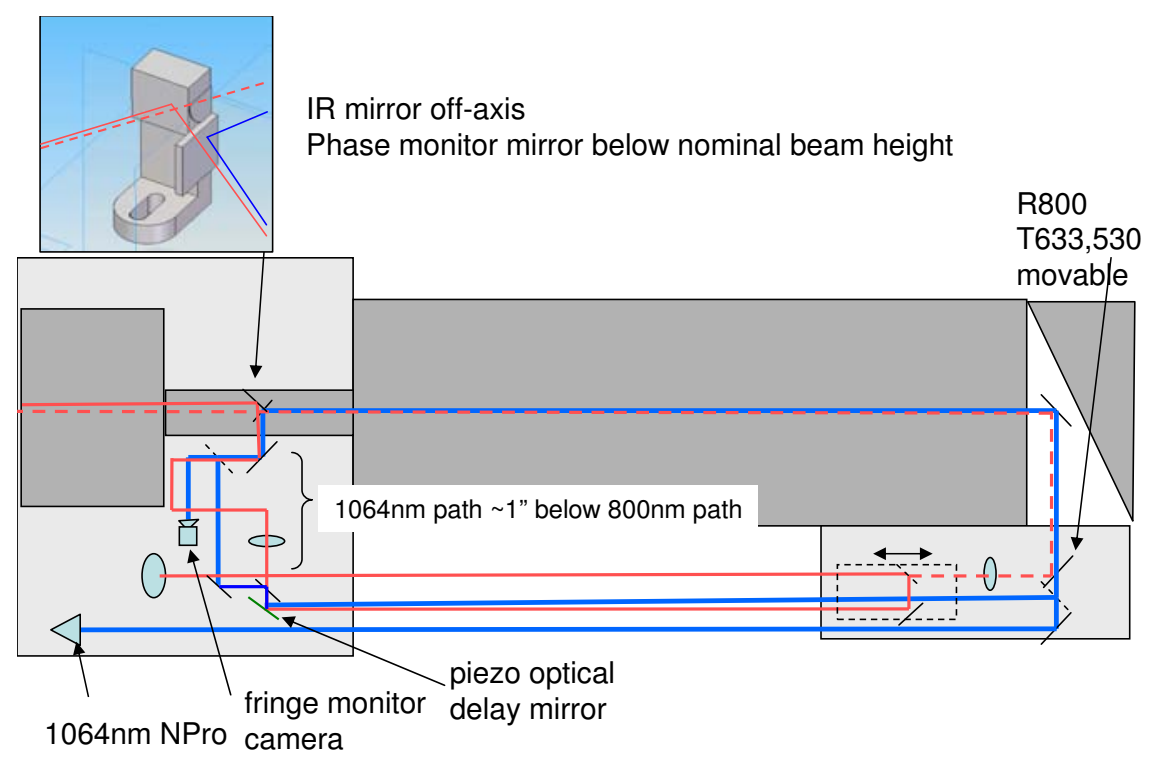

Figure 7.5: Layout of the phase monitor interferometer along side the $800 \mathrm{~nm}$ optical path. In the section of the ITR steering and focusing optics, the phase monitor optical path is positioned $\sim 1$ " below the $800 \mathrm{~nm}$ optical path. Two pick-off mirrors, one after the piezo delay mirror and the other inside the 4.5" cube, separate the monitor laser from the $800 \mathrm{~nm}$ laser path so that the two arms can be recombined and interfered on the monitor CCD.

\subsubsection{IFEL-ITR Phase Stability}

Prior to beam experiments, another CCD was temporarily placed inside the experiment chamber to observe interference fringes from the IFEL and ITR lasers in order to determine the phase stability of the optical arrangement. Comparisons were made between the phase of the $800 \mathrm{~nm}$ light to that of the phase monitor in order to determine potential differences. In particular, although the paths of the two interferometers are similar, several of the actual optical elements are different. These optics would not likely vibrate with the same frequency and phase and thus the two interferometers observe different fast jitter.

While the first measurements did observe fringes, the fringes jittered significantly. Several efforts were made to reduce noise in the interferometer paths. All optical 
mounts were converted to 1" pedestals, the dogleg breadboard was redesigned, and the vacuum roughing pumps isolated on pads. A secondary pumping cart was left off and during experiments the ventilation fan switched off. Finally, covers were installed around the optical tables and a duct installed between the two tables to eliminate turbulence from air currents in the experimental hall.

All of the efforts dramatically reduced the noise observed in phase measurements. The fast jitter which was initially found to be $>100^{\circ}$ of phase at $800 \mathrm{~nm}$ was reduced to $13^{\circ} \mathrm{rms}$ and only $6^{\circ} \mathrm{rms}$ for the phase monitor. Meanwhile the slow drift, which often could be a full $2 \pi$ of phase shift over a 5 minute period was reduced also to $40^{\circ}$. More importantly, the phase monitor was found to track quite well with the $800 \mathrm{~nm}$ phase for few minute time scales. Eventually, the phase of the experiment laser would drift away with respect to the monitor, but this occurred on time scales long enough that the experimental data itself could be used to track the drift. This will be described later in more detail. Figure 7.6 shows an example phase stability measurement comparing the phase monitor and phase of the laser to the experiment. Shown on the left is the correlation plot between the phase monitor interferometer and the primary laser for the experiment during an active sweep of the phase using the optical delay mirror. The phase of the primary laser at $800 \mathrm{~nm}$ changes over a greater range than the monitor laser at $1064 \mathrm{~nm}$. The slope of the correlation is simply the ratio of the wavelengths: $1064 / 800=1.35$. The phase offset is arbitrary, and will change with small tweaks of laser alignment or coarse delay, or from choice of ROI on the monitor CCDs.

During a measurement of net acceleration this jitter will cause a smearing out of the signal. From averaging over several samples, we can write an attenuation factor due to phase jitter as:

$$
\begin{aligned}
\delta_{p . j .} & =\frac{1}{\sqrt{2 \pi} \sigma_{\theta}} \int_{-\infty}^{\infty} \exp \left[-\frac{\theta^{2}}{2 \sigma_{\theta}^{2}}\right] \cos \theta d \theta \\
\delta_{p . j .} & =\exp \left[-\frac{1}{2} \sigma_{\theta}^{2}\right]
\end{aligned}
$$

As mentioned, the residual fast phase jitter is $\sigma_{\theta} \sim 13^{\circ}$. We then have $\delta_{p . j .}=0.97$. Thus, the fast phase jitter does not cause significant degradation to the measured 

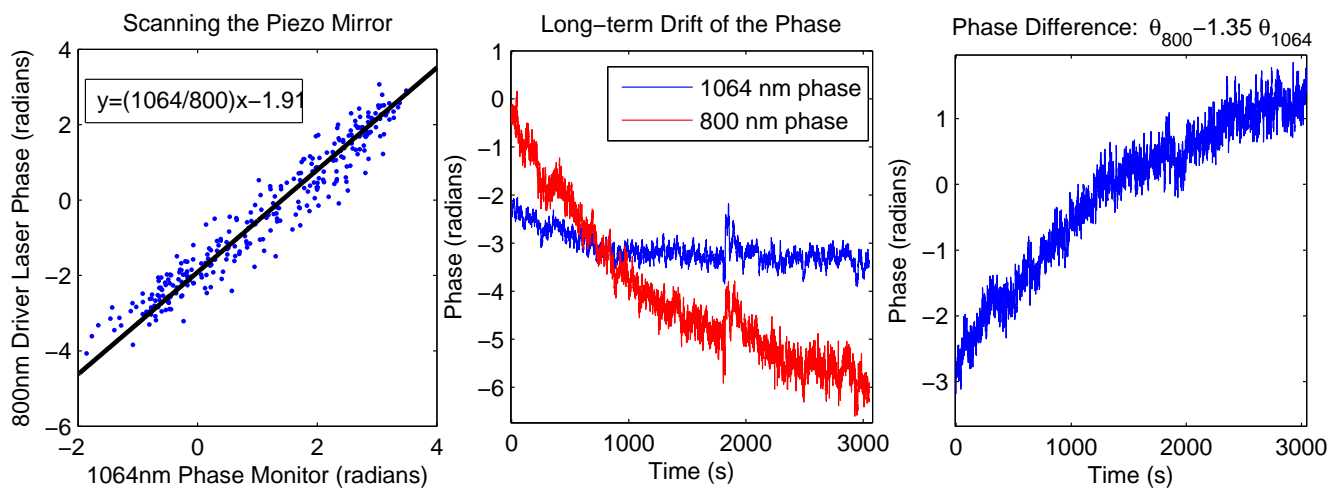

Figure 7.6: Phase Stability of the Net Acceleration Experiment. The figure on the left shows the linearity of the phase monitor to the experiment laser phase while the phase is actively scanned using the piezo delay mirror. When the phase is fixed the slow drift of the phase can be observed. Over time the experiment laser phase can wander away from the phase of the monitor system. The fast jitter is $13^{\circ} \mathrm{rms}$.

interaction.

\subsection{Results}

Attempts at the experiment were performed in two, three-week runs of the accelerator. During the first run, a few hints of net acceleration were observed, though nothing conclusive. The experience gained lead to two improvements for the second attempt. A CMOS camera sensor (without a lens) was installed directly in the path of the lasers inside the experimental chamber in order to observe fringes due to interference between the IFEL and ITR lasers. This allowed adjustment of the ITR laser steering to obtain vertically aligned fringes. Failure to obtain this alignment precisely can lead to electrons of different acceleration phase passing through the vertical slit after the ITR tape, reaching the energy spectrometer, and diluting the measured energy shift.

The second improvement was implemented in the data gathering methodology. Instead of scanning the optical delay randomly, as is typically done in the laserelectron cross correlation scans using the voice coil driven fast delay mirror, the optical delay was scanned as a sawtooth function in time with a frequency of $0.1 \mathrm{~Hz}$. Since the acceleration is sinusoidal in the optical delay, the expected signal would 
therefore roughly be sinusoidal in time. A Fourier transform of the electron beam energy would then show strong content at the drive frequency of the optical delay mirror: $0.1 \mathrm{~Hz}$. This data gathering technique proved invaluable in first observing the small net acceleration signal from the noise. Many of the data runs gathered only showed convincing net acceleration through the frequency analysis.

As mentioned earlier, evidence of net acceleration shows up not only as a shift in the centroid energy of the beam as a function of phase, but also in the beam energy spread and spectral asymmetry. For each electron energy spectrum acquired, a linear baseline subtraction is performed and the full-width half-maximum (fwhm) is calculated. This includes linear interpolation between points about the half-maximum of each side of the spectrum. At the same time as the fwhm calculation, the asymmetry is defined from the two half widths as: (lwhm-uwhm)/fwhm where lwhm is the low energy width at half-maximum and uwhm is the high energy width at half-maximum. The centroid of the energy distribution is then calculated as the mean of the spectrum ignoring portions of the spectrum with amplitude $<5 \%$ of the peak value. This cut-off reduces the influence of noise in the CCD image on the centroid calculation. Analysis of uninteracted, 'laser off' data show that the cut-off in the energy spectrum reduces the rms spread in the centroid jitter by a factor of 2 .

Although the phase monitor tracks well with the actual experiment phase in the short term, there remains the potential for slow drifts over the course of a run, especially if the run is longer than a few hundred events. Although this drift could wash-out the correlation plot between phase and the net acceleration signals, the frequency domain signals would only experience a small broadening of the peaks due to the drift. The frequency domain is therefore the best place to start in looking for net acceleration.

Figure 7.7 shows the frequency domain analysis of one of the net acceleration data runs. The transform used on the data is a brute-force discrete Fourier transform (DFT) as opposed to an FFT because of non-uniformities in the time spacing of the data. Although the electron beam and laser both arrive at a steady $10 \mathrm{~Hz}$ repetition rate, the rate of interacted events is irregular due to several factors including bucket hopping of either laser, intentional laser off data acquired for baseline analysis, or low 
charge thresholding of the data gathering.
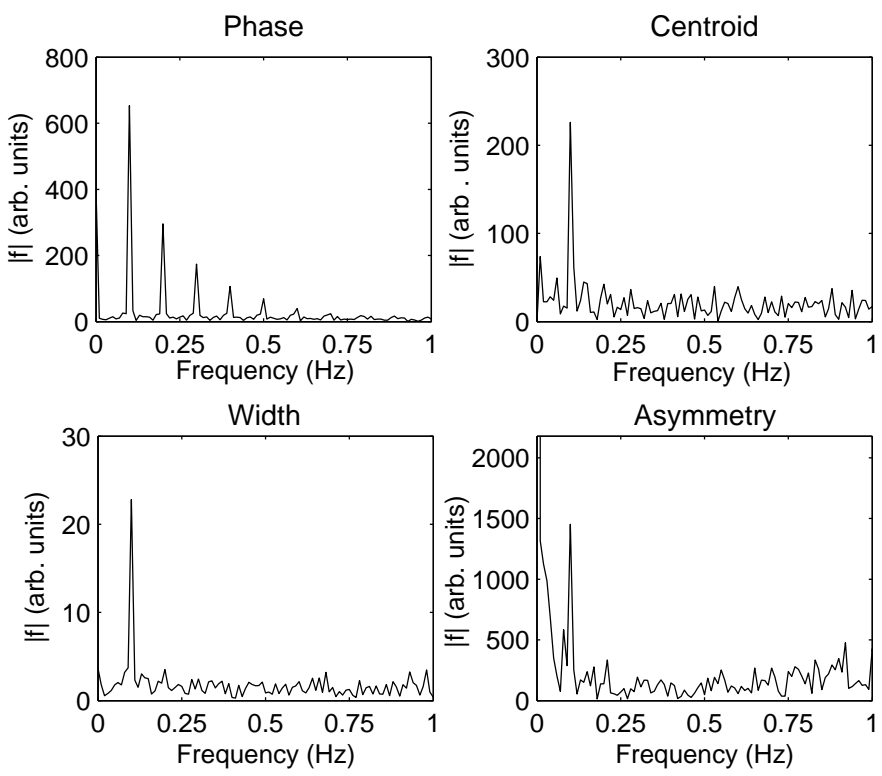

Figure 7.7: Net acceleration signal in the frequency domain. The phase (top left) is driven by a saw-tooth waveform with a $0.1 \mathrm{~Hz}$ period. The energy spectrum quantities - centroid, width, and asymmetry - sinusoidal in the phase, each show response at the drive frequency.

The top left plot in figure 7.7 shows the DFT of the phase monitor signal. Since the phase is adjusted as a saw-tooth function, the transform shows many harmonics of the $0.1 \mathrm{~Hz}$ drive frequency. The energy spectra quantities - centroid, energy width, and asymmetry - show primarily a single peak at the $0.1 \mathrm{~Hz}$ drive frequency. Because of the phase reset inherent in the saw-tooth waveform, the variation of the net acceleration is not strictly sinusoidal in time. As a result, occasionally a second harmonic can be seen in the net acceleration DFT data. More often though, this effect is hidden in the noise of the DFT.

Although the DFT plots are direct evidence that net acceleration is occurring, observing the correlation between phase and the centroid energy directly would be a more fitting demonstration. As mentioned earlier, during long runs, the phase offset between the experiment and the phase monitor can slowly drift. Fortunately, the data itself can used to correct for this slow drift. During data analysis, it was found that 
the energy spread oscillation as a function of phase had the best signal-to-noise ratio. In hindsight, this is not a surprise since the interacted energy spread is easily 3 times larger than the initial energy spread and more than 10 times larger than the energy spread jitter (typ. $\sim 5 \mathrm{keV}$ ). The net acceleration data for each run is subdivided into sets of 500 events and the offset of the energy spread versus optical phase calculated from an FFT. The top left plot in figure 7.8 shows the wander of this phase offset as a function of time. For this particular run the wander is almost a full $2 \pi$ of shift over the entire 14 minute long run. This drift data is then used to correct the phase for the entire data set before plotting the energy centroid, spread, and asymmetry versus optical phase.
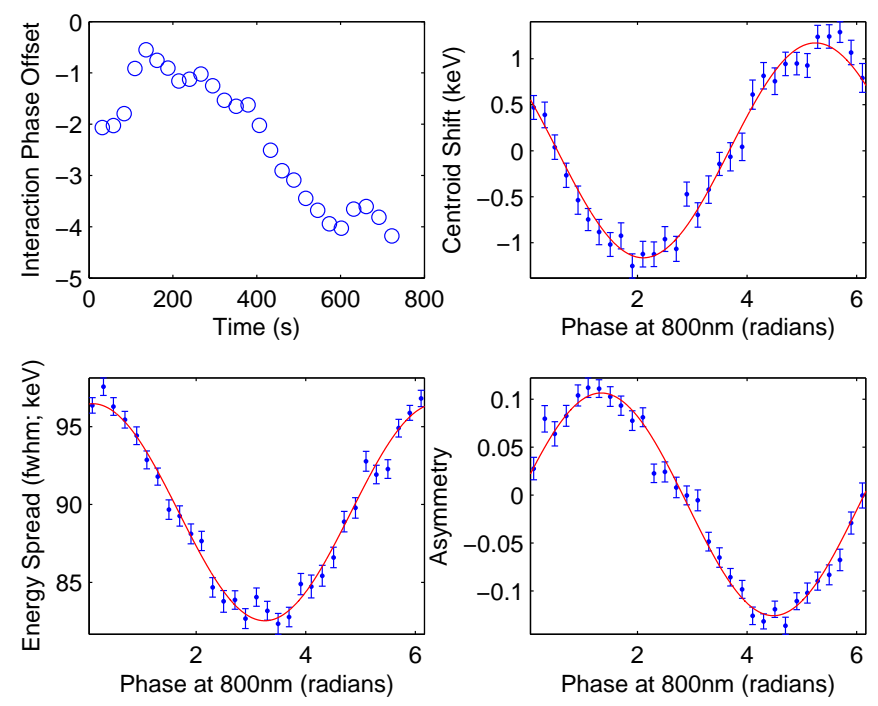

Figure 7.8: Correlation of phase with the net acceleration signals (Run 3661). The top left panel shows the slow drift of the experiment phase offset relative to the phase monitor interpreted from the phase versus energy spread data. The remaining three plots are the binned data after correcting for the slow drift. The error bars are the deviations of the means for the binned data.

The data for the three correlation plots shown in figure 7.8 are binned and plotted along with a fit to a cos function. Cuts are applied to remove laser off and 'bucket-hop' events where no laser-electron interaction occurs. The error bars are the standard deviations of the means for each bin. Notice, as predicted by the Monte 
Carlo simulations, the asymmetry is $\pi$ out of phase with respect to the centroid while the energy width is $\pi / 2$ out of phase. What do not agree are the amplitudes of the fits. For all three the amplitude is less than expected, especially the centroid energy shift. Prior to this run, the IFEL and ITR interactions were established separately with modulation strengths of $80 \mathrm{keV}$ and $35 \mathrm{keV}$ respectively and an initial energy spread of $47 \mathrm{keV}$ fwhm without laser interactions. This gives an average bunching factor for the beam of $b_{1}=0.35$ (eqn. 2.31) and an expected centroid shift of $b_{1} \times 35 / 2=6 \mathrm{keV}$. This compares to a measured amplitude of just $1.2 \mathrm{keV}$.

All-told, 58 data samples showing net acceleration signal were obtained over two weeks of run time. The ITR laser alignment was changed several times and the phase fringe alignment checked with the in-vacuum CMOS camera. Some runs obtained somewhat stronger ITR interactions, up to $50 \mathrm{keV}$ fwhm modulation. However, the measured signal never got much above $2 \mathrm{keV}$ in amplitude, well short of the expected signal. It is possible this may indicate a fundamental problem in the experiment setup, though without further study it will remain unclear.

\subsection{Conclusion}

Despite the total measured centroid shift failing short of the expected signal, the experiment showed great success simply by measuring net acceleration. This is the first demonstration of direct optical acceleration at visible wavelengths. The experiment explored many of the important aspects of direct laser acceleration including staging of two optical accelerators. A key aspect of this experiment was the optical phase monitoring and control. Great effort was needed to reduce phase noise and drift.

Future experiments with a stronger second stage interaction will be needed to further explore issues such as charge capture. The small centroid shift compared to the induced energy spread makes defining the captured charge from the energy spectrum very difficult. 


\section{Chapter 8}

\section{Tight Focusing of Electron Microbunches}

This chapter presents work begun during the graduate term in producing tight electron focuses for injection into optical scale accelerator structures. Few micron scale spot sizes are produced using a permanent magnet quadrupole (PMQ) triplet. Although a separate goal from the production and acceleration of optical microbunches, the tight focusing will nevertheless be a vital step in demonstrating efficient acceleration at optical wavelengths. Hardware design and fabrication as well as significant simulation work were done and are presented. Unfortunately, due to delayed commissioning of the E163 facility, time was not available to begin experiments using the PMQ triplet for inclusion in this thesis.

\subsection{Optical Scale Structures And Tight Focusing}

To date all laser acceleration experiments have utilized largely free space interactions where the laser propagates free of any guiding structures besides mirrors for bringing the laser in and out of the beam path. The ITR experiment did introduce a single boundary to terminate the laser and provide net acceleration, however the laser still propagated as a free space Gaussian mode. Keeping the laser in free space has obvious advantages. The optical components are macroscopic objects, easily designed 
and installed. Alignment can be done with standard scientific cameras and imaging techniques. However, there is a significant drawback in that the electric field of a free space laser is primarily transverse and not useful for acceleration. The electrons must be coupled to this electric field. The IFEL does this by introducing a transverse motion to the electrons, the ICA and ITR schemes both bring the laser in at a small angle compared to the electrons. In any case, either the amount of electric field accelerating the electrons is small compared to the total peak field or the overall interaction length is short. This leads to a weakly coupled acceleration mechanism, or a small interaction impedance. The interaction impedance is defined as the accelerating field (or gradient) divided by the total power in the accelerating field (equation 8.1).

$$
Z_{\text {int }}[\Omega] \equiv\left|\lambda E_{0}\right|^{2} / P
$$

For a simple comparison consider a $1 \mathrm{~mJ} /$ pulse picosecond laser (=1 GW power) focused to a few hundred microns $\left(E_{\text {peak }} \approx 400 \mathrm{MV} / \mathrm{m}\right)$. For an IFEL interaction (see §2.1) $Z_{\text {int }}$ is typically $O\left(10^{-8}\right) \Omega$. For ICA and ITR the effective gradient is given by $E_{\text {peak }}$ over the angle between electron and laser which is optimally $1 / \gamma$ giving an interaction impedance of $Z_{\text {int }} \sim 10^{-7} \Omega$. By comparison optical scale structures have much higher interaction impedances, ranging from a few ohms to hundreds of ohms[65, 66, 67]. This means that the energy efficiency is far greater for optical scale structures. The challenge of course with optical scale structures is their size. Fabrication, system integration, and beam coupling, both electrons and optical, are all much more difficult than in previous experiments.

The challenge of coupling the electron beam into such small structures can be addressed by using a very strong focusing element close to the optical scale structure. Such a focus element has been designed and fabricated for the E163 program. The device uses three magnetic quadrupoles made with permanent magnets. It can be used in conjunction with the microbunching hardware to deliver optically spaced electrons to laser accelerators or on its own in laser modulation or beam wakefield experiments. Section 8.2 explains in further detail the focusing characteristics needed in order to couple to optical scale structures, deriving a few simple equations describing focusing. 
Section 8.3 shows the simulation and design work and subsequent measurement of the PMQ triplet. Field measurements found the PMQ triplet to have some surprising field errors, though particle tracking shows these errors to be manageable and allow the necessary focusing. The final section of this chapter discusses the first optical scale accelerator experiment. Using the PMQ triplet, the beam will be focused into an optical scale structure and the induced wakefields observed spectroscopically.

\subsection{Beam Transport and Focusing}

Conventional electron optics prove insufficient to obtain the small spot necessary for transmission through an optical structure. The chromatic aberrations for a long focal length triplet prevent reaching spot sizes much below 100 $\mu \mathrm{m}$ in the E163 beamline where as the structures under investigation for optical acceleration typically have apertures of only a few microns. Without tighter focusing, the transmitted electrons would be too few to reasonably detect with currently installed diagnostics.

In order to quantify the focusing characteristics necessary for this coupling problem we turn to the well used language of Twiss parameters to describe the electron beam $[68,69]$. Of interest here are $\beta$, describing the transverse envelope of a beam such that $\sigma=\sqrt{\beta \epsilon}$ where $\sigma$ is the spot size and $\epsilon$ is the emittance of the beam. The beam emittance $\epsilon$ will often vary depending on, for instance, the electron source. The Twiss parameter $\beta$ however is a property only of the transport lattice (i.e. magnets). When a beam is focused there occurs a non-zero minimum in $\beta$ at the focal location, termed $\beta * . \quad \beta *$ is analogous to the Raleigh range for a focused laser. For optimal transmission through a fiber $\beta *$ must equal half the length of the fiber. For structures being considered for the E163 experiment this means $\beta *$ in the range of 0.5 to $2.5 \mathrm{~mm}$. Equation 8.2 gives the relation for $\beta *$ as a function of the focal length of the triplet $f$, the energy spread $\sigma_{p} / p$ and the initial $\beta_{0}$ [70]. Figure 8.1 shows the focusing characteristics of two triplets.

$$
\frac{\beta *}{\beta_{0}}=\frac{1+\left(\beta_{0} / f\right)^{2}\left(\sigma_{p} / p\right)^{2}}{1+\left(\beta_{0} / f\right)^{2}\left[1+\left(\sigma_{p} / p\right)^{2}\right]}
$$




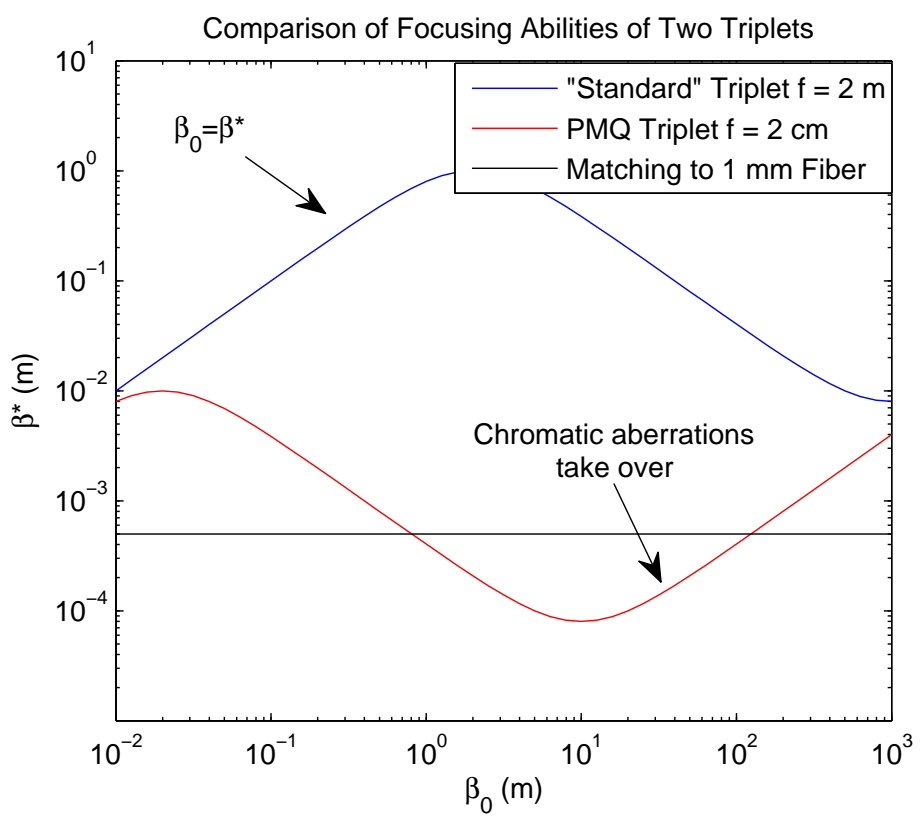

Figure 8.1: plot of equation 8.2 with two focal lengths, and $0.2 \%$ energy spread.

From figure 8.1 we see that for a standard triplet chromatic effects take over before a sufficiently tight focus is reached. A short focal length permanent magnet quadrupole however can obtain the necessary focusing. The total transmission can then be determined by comparing the transmitted emittance, or acceptance, of the structure to the initial emittance. The acceptance is determined by the allowable range of position and angles that can pass through the structure, as illustrated in figure 8.2. For a $1 \mathrm{~mm}$ long fiber with a $5 \mu \mathrm{m}$ aperture the transmitted emittance is $0.03 \mathrm{~mm}-\mathrm{mrad}$ (geometric). This compares quite favorably to the expected incident emittance of $0.03 \mathrm{~mm}-\mathrm{mrad}$ RMS at E163. Given clipping in both transverse dimensions this gives an acceptance of $\sim 50 \%$.

\subsubsection{Washout of a Tightly Focused Microbunched Beam}

Though initial experiments using the PMQ triplet will use an unbunched beam, in future experiments investigating acceleration from powered optical scale structures it may be of interest to use a microbunched beam produced by the microbunching 

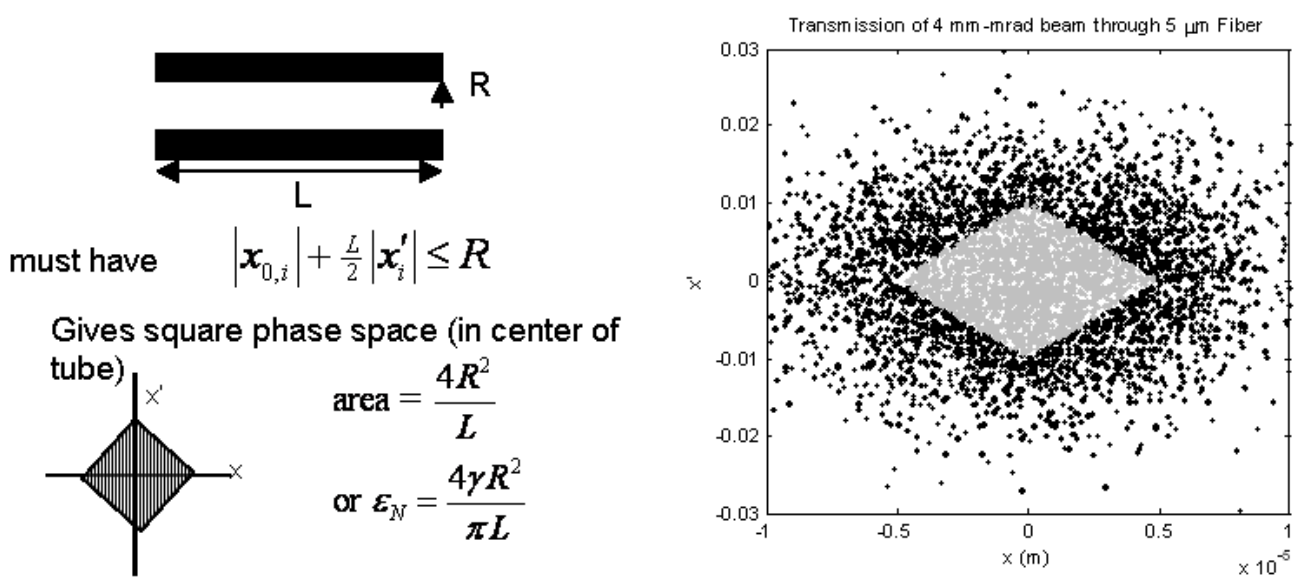

Figure 8.2: Transmission phase space of a one dimensional structure. The initial and final aperture of the structure limit the total phase space area of the beam. The plot on the right shows the phase space halfway through the structure.

hardware. However, first we must know whether the microbunch structure will survive the strong focusing imparted by the PMQ triplet. As described in section 2.4.1, path length differences due to particle divergence angles can washout the microbunch structure. In the case of the PMQ triplet, each particle attains a focusing angle given by $\theta \approx r / f$ where $r$ is particle distance from the beam center and $f$ is the focal length of the triplet. For a beam with $\sigma_{r}=100 \mu \mathrm{m}$ the rms angular spread of the beam is then $\sigma_{\theta} \approx 5 \times 10^{-3}$ radians. Using equation 2.38 we have $\delta_{\theta, 1}=0.57$ and $\delta_{\theta, 2}=0.47$. Thus, we see that the focusing causes significant degradation of the microbunches. Of course, this simple picture treats the PMQ as a thin lens, which is in reality far from the true case.

The degradation is worse when we take into account the path length differences of particle trajectories within the PMQ triplet itself. For this we turn to a numerical approach that integrates the trajectories through the triplet to find the slippage $\delta_{z}$ relative to the centroid trajectory as a function of transverse offset: $X_{0}, Y_{0}$. Figure 8.3 illustrates the effect of the particle transverse position on the final longitudinal position. Since the PMQ triplet is initially defocusing horizontally, the slippage is worse for particles with large horizontal offsets. 

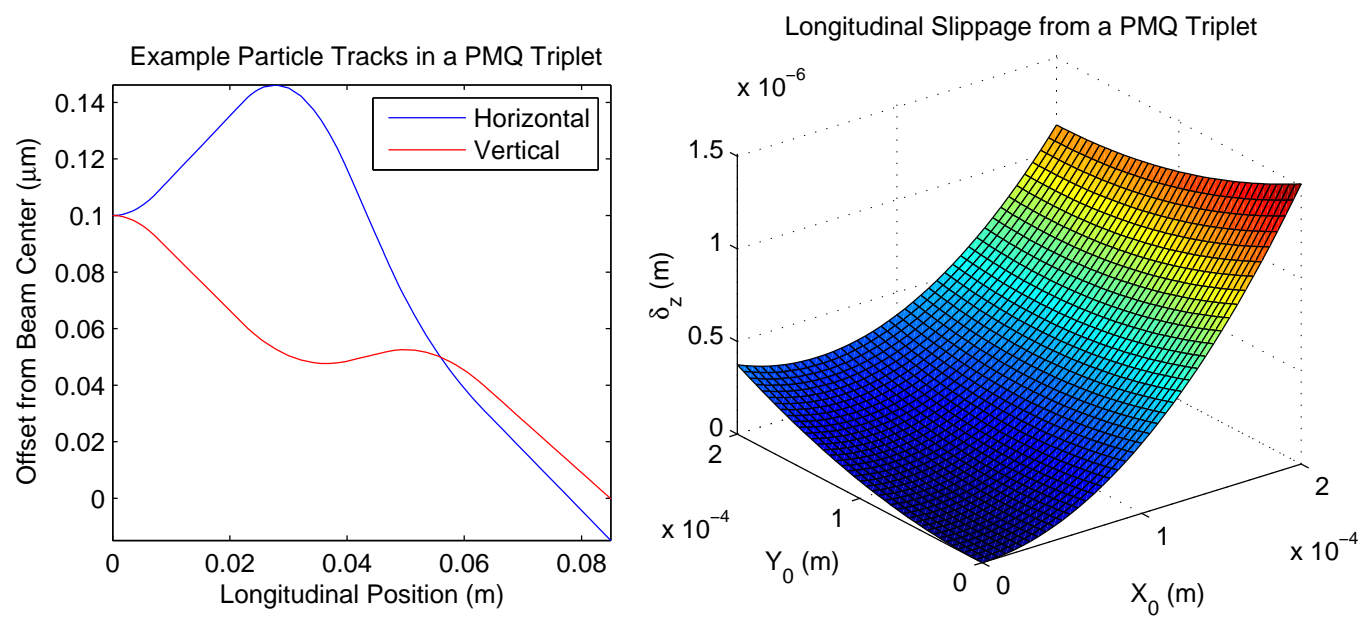

Figure 8.3: Slippage of particle trajectories relative to the initial particle position entering the triplet. Left are two particle trajectories for a horizontally (blue) and a vertically (red) offset particle. The triplet is initially defocusing horizontally. The figure on the right shows the slippage $\delta_{z}$ relative to the beam centroid as a function of transverse position.

For a microbunched beam, the portion of the beam with an initial transverse position $<X_{0}, Y_{0}>$ entering the triplet will form at each harmonic of the microbunching a phase shift of $\delta_{\theta, n}=\cos \left[n k_{l} \delta_{z}\left(X_{0}, Y_{0}\right)\right]$. We can average this over a beam with an rms spot size $\sigma_{x}=\sigma_{y}$ to obtain attenuation terms for each harmonic analogous to equation 2.38. We find for $\sigma_{x}=\sigma_{y}=100 \mu \mathrm{m}$, we have $\delta_{\theta, 1}=0.26$ and $\delta_{\theta, 2}=0.08$. As expected, this is even worse than the initial guess assuming a thin lens. Figure 8.4 shows the degradation of the microbunching from focusing in the PMQ triplet as a function of the initial transverse spot size.

\subsection{Designing the Permanent Magnet Quadrupole Triplet}

The triplet design began by using Elegant[43] simulations to find the strength of quadrupoles necessary to obtain sufficient focusing of the beam. Initial Radia designs began with a simple quadrupole consisting of four magnet blocks and four iron 


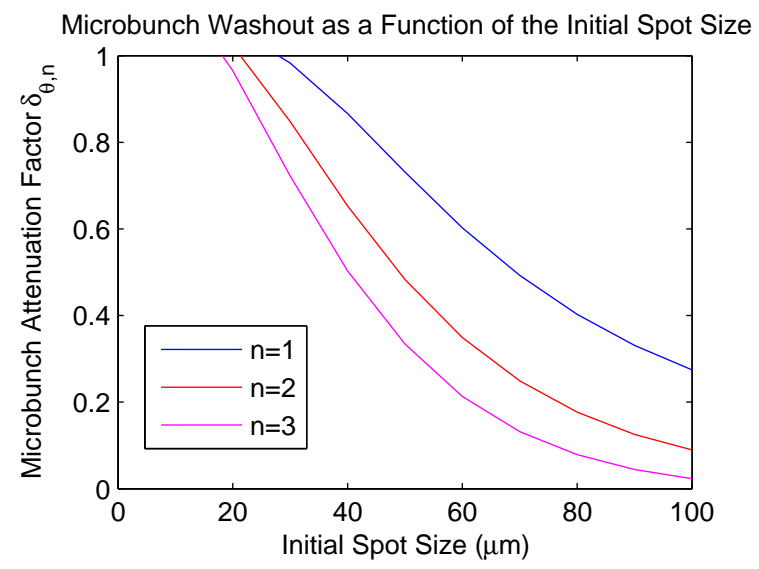

Figure 8.4: Microbunch attenuation factors due to focusing in the PMQ triplet.

pole pieces (see figure 8.5). This was compared to another recently designed PMQ triplet[71] that used the Halbach geometry with 16 magnet pieces of varying polarization and no iron[44]. It was found that the hybrid magnet/iron pole arrangement had several advantages. For the same aperture, the hybrid arrangement has a quadrupole strength $\sim 25 \%$ greater than the Halbach design. This is due to the greater saturation field sustainable in the iron compared to the residual field of the NdFeB material. In addition, the Halbach arrangement uses 16 magnet blocks with 5 different polarizations compared to just 4 blocks with only one polarization in the hybrid geometry. This helps simplify fabrication. The use of a saturated iron pole tip also helps reduce the influence of magnet block errors on the final field quality.

Much like the undulator, the peak field can be increased by shortening the pole tips relative to the magnet blocks. Other designs[72] have further increased the field by adding magnets radially above the iron pole tips, however this was found to be an unnecessary complexity for this project. Additional refinements could include shaping the poles to minimize higher order components ${ }^{1}$ However, since this triplet will act only as a single pass device, and the higher order components are very small even for a flat pole tip, the poles were kept flat to simplify fabrication. Table 8.1 lists the

\footnotetext{
${ }^{1}$ An interesting side note, during simulation it was observed that the next order field component in the quadrupole design, the dodecapole, can actually help cancel chromatic aberration and reduce emittance growth. However, as noted in the above text, this was not strongly pursued since tayloring the pole tips would add significant complexity to the fabrication.
} 

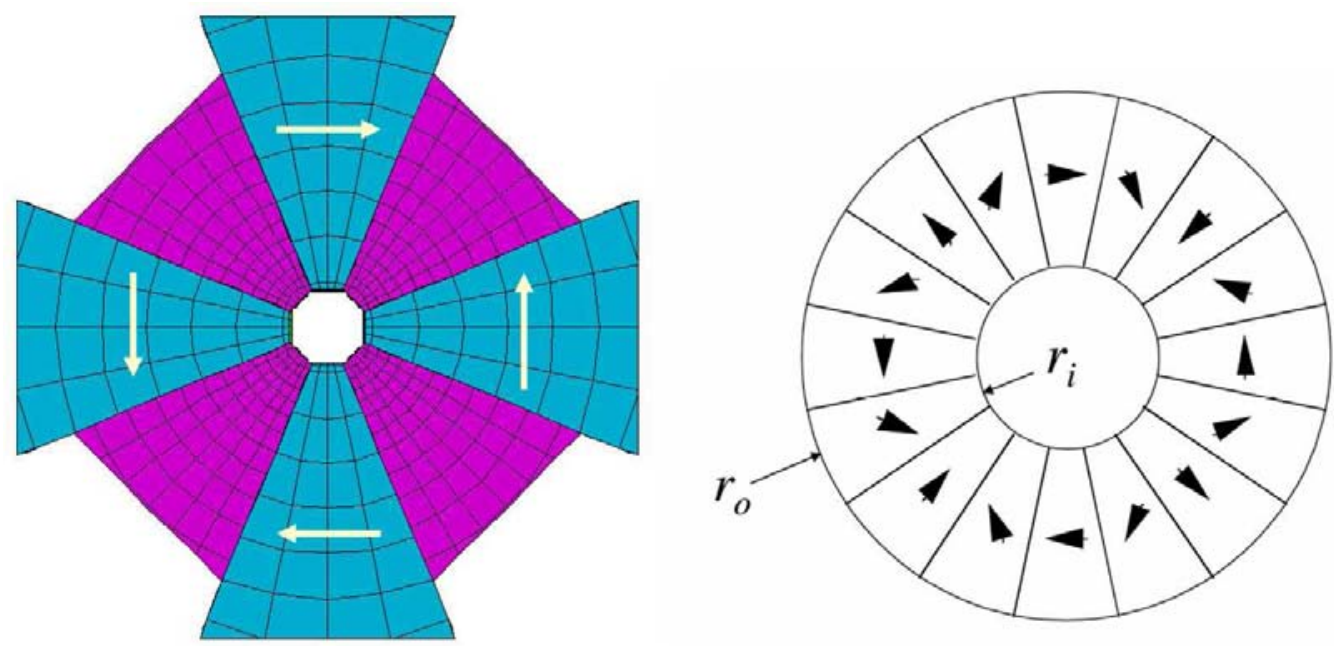

Figure 8.5: Left: Radia CAD image of Permanent Magnet Quadrupole (Blue=Magnet, Magenta=Iron). The arrows show the magnet polarization. Right: Halbach arrangement for a PMQ.

design specifications for the triplet. The quadrupole widths are chosen such that the integrated field strengths follow a 1-2-2 design ratio for triplets. The triplet can be adjusted by changing the spacing between each PMQ. For focusing a $60 \mathrm{MeV}$ beam the spacings are about $15 \mathrm{~mm}$ each.

\begin{tabular}{|l|c|}
\hline Parameter & Value \\
\hline Gap & $3 \mathrm{~mm}$ (center to corner) \\
Magnet Thickness & $20 \mathrm{~mm}$ \\
Magnet Material & $\mathrm{NdFeB}, B_{r}=1.25 \mathrm{~T}$ \\
Pole Thickness & $16 \mathrm{~mm}$ \\
Pole Material & $1010 \mathrm{Steel}$ \\
$1^{\text {st }}$ quad width & $7 \mathrm{~mm}$ \\
$2^{\text {nd }} \& 3^{r d}$ quad width & $13 \mathrm{~mm}$ \\
Quadrupole spacings & adjustable $4-20 \mathrm{~mm}$ \\
\hline
\end{tabular}

Table 8.1: List of PMQ design specifications.

The assembly for the triplet proved far more complicated than for the undulator or chicane (§3.5). Not only does the triplet require separate motion for all three quads, the alignment tolerances are also more stringent, especially the alignment of the quads relative to one another. Rotation, or skew, errors can lead to emittance growth while 
translational offsets cause strong kicks to the beam. To fix the alignment, each quad moves on a pair of steel guide rods. The $3^{\text {rd }}$ quad is fixed to a common optical stage that can move all three quads in union. The first two quads are also movable independently by stepper motors. $5 \mathrm{~mm}$ stoppers prevent the quads from coming completely together, at which point the magnetic forces would prevent separating them. Springs are also positioned around the guide rails to assist separating the quads when the gap is small. Figure 8.6 shows the fully assembled PMQ triplet.

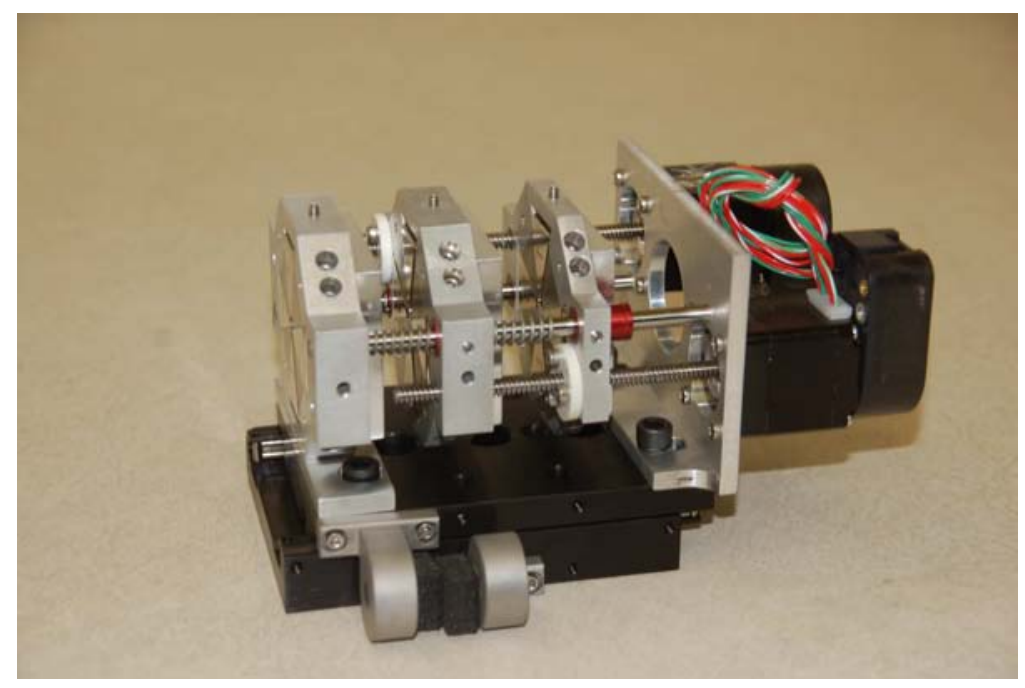

Figure 8.6: The Permanent Magnet Quadrupole Triplet. Not shown is a third motor needed to drive the optical stage.

\subsubsection{PMQ Measurement}

Unlike the undulator and chicane which could easily be measured using a standard Hall probe and linear motion stages(§3), the small aperture of the PMQ made measurement difficult. The Hall probe or its supporting arm often collided with the PMQ assembly. There was even less room to move the probe transversely, a necessary step in measuring the quadrupole gradient. It is also very difficult to obtain information on higher order field content from a Hall probe. Despite this, an initial attempt to measure the PMQ fields was done with a Hall probe. Two sets of scans were performed using an axial and a transverse probe. The axial probe scans near the pole tips show 
surprising non-zero axial field in the mid-plane of the quadrupoles (see figure 8.7). This implies that the transverse field under the pole is not longitudinally symmetric.
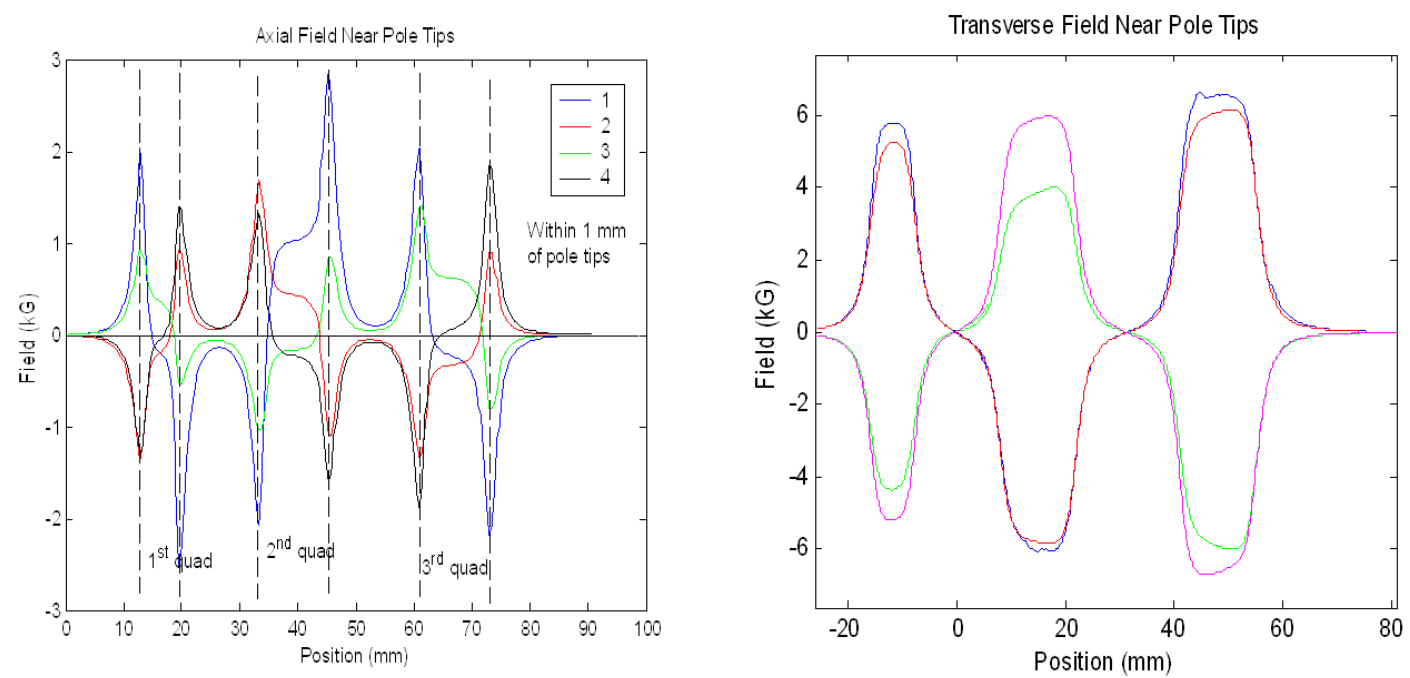

Figure 8.7: Hall probe data of triplet. Notice the non-zero axial field at the center of each quad. Each color represents different set of pole tips. Due to alignment difficulties, the separation of the probe off of the pole tips for each scan was not consistent. Thus, only the relative shapes are of interest in these figures and not the total scale.

A later check with a transverse probe did find this to be the case. However, because of the limitations found with the Hall probe, further measurements relied on different techniques.

\section{The Pulsed Wire Scan}

The pulsed wire scan[73] involves stringing a small (0.0015") diameter wire through the aperture of the PMQ triplet. A short, high voltage pulse is sent along the wire. The current interacts with the magnetic field and causes a force on the wire. This in short order causes a displacement which then travels down the length of the wire. The wire displacement is read out to determine the profile of the magnetic field. The wire displacement is actually proportional to the first integral of the field. Many pulsed wire measurements look only at the total integral, however by sending the signal to a scope one can get the first field integral as a function of position along the magnet. 
Furthermore, since the wire can be pulsed several times a second, it is easy to average over many shots to remove noise. The derivative can then be taken to get back the initial field. Since derivatives of scope traces are often noisy, an improvement to the setup was to add an $\mathrm{RC}$ differentiator prior to the input to the scope. The time constant of the $\mathrm{RC}$ circuit is chosen such that the maximum frequency of interest in the scan was much less than one over the time constant of the differentiator circuit. The pulsed wire technique has many advantages of the Hall probe most notably in speed since an entire longitudinal scan is done simultaneously. Additionally, by setting up two orthogonal detectors monitoring the wire the horizontal and vertical fields are detected at the same time.

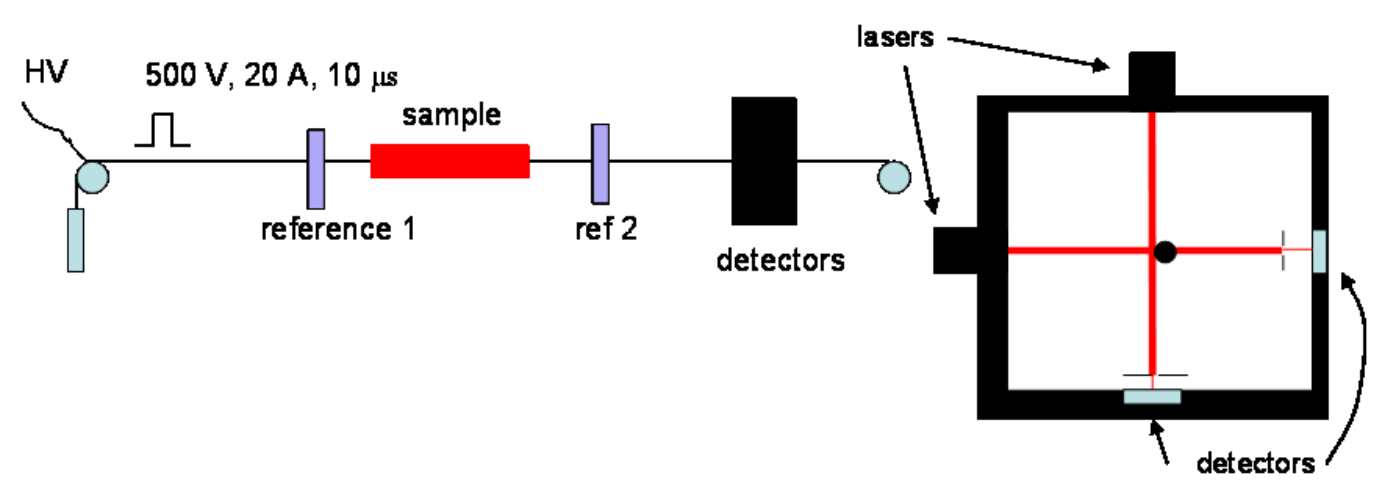

Figure 8.8: Schematic of pulse wire setup. Entire length is 1 meter. Wire diameter is $0.0015 \%$.

The almost instantaneous readout of both transverse field components for the entire length make alignment very quick and easy. By aligning the PMQ assembly to zero the signal from the first and last quads, we find a displacement error on the middle quad of $-43 \mu \mathrm{m}$ horizontally and $93 \mu \mathrm{m}$ vertically. Previous beam simulations showed emittance growth is negligible for these displacements. To first order, the only change to the beam is a steering kick, $-1.5 \mathrm{mrad}$ horizontally and $+3.6 \mathrm{mrad}$ vertically. This can be corrected for using steering magnets upstream and downstream of the experiment.

Another check easily performed with the wire scan is a rotation error of a quadrupole about the nominal axis of the PMQ triplet. Such an error produces a skew field that 
cross-couples the transverse phase spaces and leads to an increase in both projected emittances. The tolerance for the rotation error is very tight; an error of $10 \mathrm{mrad}$ will cause a $33 \%$ increase in emittance. To check for rotation error, we compare two scans of $B_{y}$ at transverse positions $(1,1) \mathrm{mm}$ and $(1,-1) \mathrm{mm}$. For no error, $B_{y}$ should be constant for changing y, any change in $B_{y}$ therefore denotes a skew term from a quad being rotated. Comparing two such traces we find there is no error to within $2 \mathrm{mrad}$ limited by the trace calibration.

There are however, several drawbacks that hinder the effectiveness of the pulsed wire scan. One is dispersion of waves on the wire. After the initial impulse from the current excitation, the acoustic wave on the wire propagates several centimeters to the detector. High frequency components travel slower with the result that ripples are seen on the trace at regions of quick field transition. The dispersion can be partially alleviated by choosing a smaller wire, though this only works to an extent and eventually smaller wires become impractical. To remove remaining dispersion, the traces can be corrected mathematically by appropriately phase shifting the transforms 2. In practice, the correct amount of shift is judged qualitatively by minimizing the ripple on a single trace and applying to the remaining data. Figure 8.9 shows the results of the dispersion compensation.

Another pitfall is the detector non-linearity. The detectors work by illuminating the wire with a laser and projecting a shadow onto a slit with a photodiode behind it. As the wire vibrates the shadow moves across the slit producing the signal which is then amplified and sent to the oscilloscope. The shadow, however, is a diffraction pattern produced by the wire, and thus the signal is a convolution of a square pulse

\footnotetext{
${ }^{2}$ The dispersion relation for a non-ideal string can be understood by first considering, a 'lumpy' string. Recall for modes on a beaded string we have: $\omega=2 \sqrt{\frac{T}{m a}} \sin \left(\frac{1}{2} a k\right)$ where $\mathrm{T}$ is the tension of the string, $m$ the mass of each bead, and $a$ is the separation between beads. So, to first order $\frac{\omega}{k}=v_{0}=\sqrt{\frac{T}{m} / l}$ - the well - known velocity of modes on a string. However, to next order: $\frac{\omega}{k}=$ $v(k)=v_{0}\left(1-\frac{a^{2} k^{2}}{6}\right)$. For a non-ideal string or wire, there exist non-uniformities much like the beads used in this derivation. We therefore expect an additional phase shift (skipping the math): $\phi(k)=c^{\prime} k^{3} L$ where $\mathrm{L}$ is the distance from magnet to the detector and $c^{\prime}$ some constant determined by minimizing ripple in the traces in a sample field profile. So to compensate we take an FFT and multiply by $\exp \left[i c^{\prime} k^{3} L\right]$ then transform back.
} 


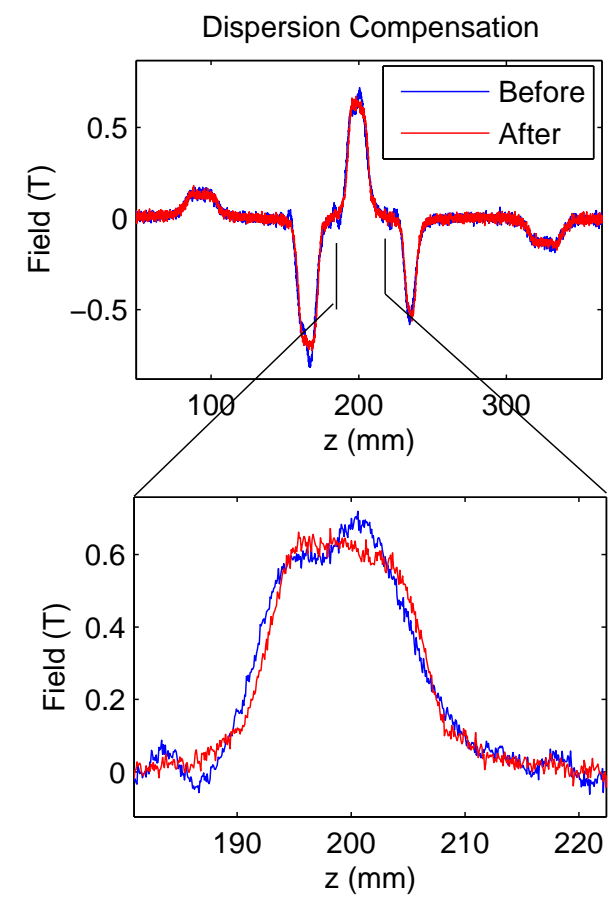

Figure 8.9: Compensation of acoustic dispersion in magnet wire scan. Above, full trace through PMQ triplet before (blue) and after (red) an fft compensation of acoustic dispersion. Below: a zoom in of the center quad. Note the ripples to the left of the peak in the blue trace.

from the slit and a sinc function rather than a trapezoid response as desired. The setup could be improved by imaging the wire onto the slit, thus eliminating the nonlinearity. This solution, however, was not pursued since by that point it became clear to best determine field quality a coil scan would work best.

\section{The Rotating Coil}

When it comes to determining the field quality of a quadrupole the best technique to use is a rotating coil. A coil is made to rotate such that one side is on the axis of rotation while the other sweeps through the magnetic field transversely. The field then induces current inside the coil related to the harmonic content of the field. A lock-in amplifier or an oscilloscope with an FFT function is then used to analyze the induced signal for the multipole content of the magnet (for a short description on the 
multipole expansion of a 2D magnet field see appendix A). Since the PMQs have such small apertures, the coil is very small, just $1 \mathrm{~mm}$ square with 10 turns. A motor turns the coil at $30 \mathrm{~Hz}$. One trouble found in the setup related to the contacts for the rotating coil. Originally, the rotating shaft had 3 pairs of contacts near the motor spaced $120^{\circ}$ apart. This turned out to introduce an intermittent sextupole term in the signal. Removing two of the contact pairs alleviated this problem.

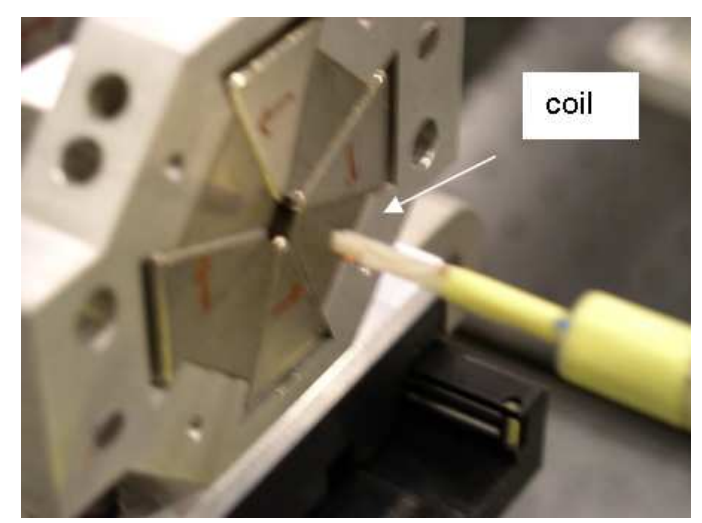

Figure 8.10: Coil tip in front of the PMQ triplet. The coil tip measures $1 \mathrm{~mm}$ by $1.5 \mathrm{~mm}$. The quadrupole aperture is $5 \mathrm{~mm}$.

An example spectrum of a PMQ is presented in figure 8.11. Misalignment of the rotating axis with respect to the quadrupole axis causes the appearance of a dipole term. This scan was taken prior to the realization of the cause and correction of the sextupole term. The most notable multipole is a decapole term that appears in all three quadrupoles. Radia simulations showed no decapole term. In fact the only significant multipole from Radia was a dodecapole term that was some 5 orders of magnitude smaller than the quadrupole term. By comparison the decapole term measured is about $5 \%$ of the quadrupole term (decapole strengths are given in table 8.2). This then presents a significant field error in the design. The source of the error remains uncertain, attempts to duplicate the error in magnetostatic simulations required unrealistic dimensional or magnetic errors. At the same time though, particle tracking simulations with the measured errors show that for spot sizes going through the triplet the focusing properties will not be too adversely affected (see figure 8.12).

The coil was also made short so that scans could be made to check for changing 


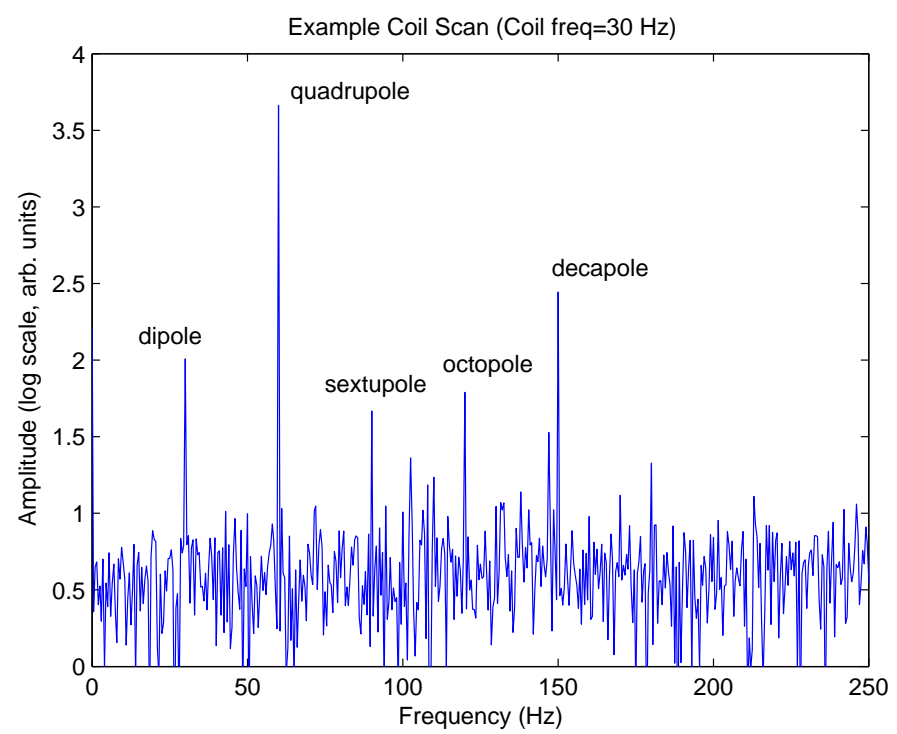

Figure 8.11: Signal from rotating coil with multipoles labeled.

\begin{tabular}{|c|c|c|}
\hline & $a_{4} / a_{1}\left(\mathrm{~mm}^{-3}\right)$ & $b_{4} / a_{1}\left(\mathrm{~mm}^{-3}\right)($ skew $)$ \\
\hline PMQ 1 & -0.022 & 0.038 \\
PMQ 2 & 0.060 & -0.0033 \\
PMQ 3 & 0.022 & 0.022 \\
\hline
\end{tabular}

Table 8.2: Decapole coefficients for each PMQ. For a description of multipole coefficients see appendix A.

multipole content longitudinally. In actual fact though, the multipole content is constant relative to the quadrupole amplitude to within the noise of the measurement.

An attempt was also made to track the phase of the multipoles, though this proved a noisy measurement, with an error of $\sim 15^{\circ}$. This could probably be improved by using a lock-in amplifier rather than an oscilloscope to measure the harmonics. 


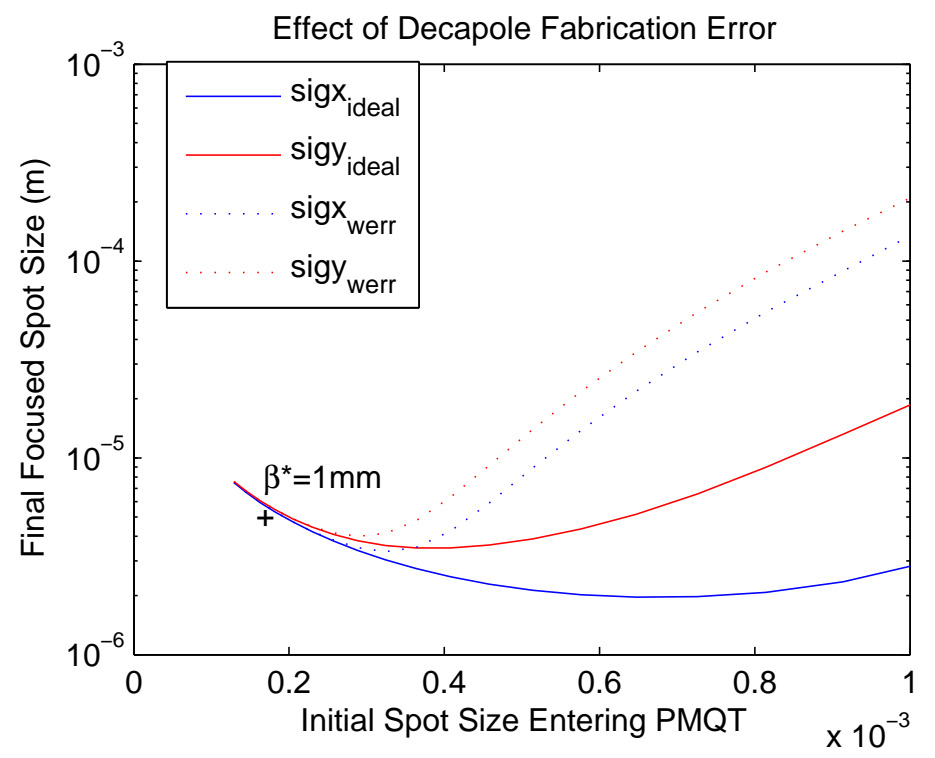

Figure 8.12: Elegant[43] simulations of the effect of the decapole term on focusing characteristics assuming a normalized emittance of $2 \mathrm{~mm}-\mathrm{mrad}$ and an energy spread of $0.2 \%$. The plot shows the focused transverse spot sizes as a function of the initial spot size before the triplet for the ideal focusing with only chromatic aberration (equation 8.2, solid lines) and for the focusing produced by the real PMQ triplet fields (dashed lines). For the region of operation around $\beta *=1 \mathrm{~mm}$ the degradation is negligible.

\subsection{Optical Wakefields From a Photonic Bandgap Fiber}

Closed metallic accelerating structures confine electromagnetic waves by definition. The design criteria for metallic structures used at RF involve obtaining speed-oflight synchronicity, and a mode with uniform longitudinal electric field at a frequency matching that provided by powering klystrons. Confining the field within the structure is never an issue. Unfortunately, at optical frequencies, absorption in metals becomes significant, far greater than dielectric materials. In addition, metals damage at much lower peak electric fields. This makes the use of dielectrics for acceleration at optical frequencies much more appealing.

In order to confine electromagnetic waves in dielectrics, the structure must rely 
on interference of waves scattered off of boundaries in the structure. The simpliest example is a dielectric mirror, a series of alternating layers of two different dielectrics. Wavelengths of light that match the Bragg condition set by the layer thicknesses are reflected away from the mirror. Such a structure is known as a photonic crystal. The range of wavelengths that are forbidden by the structure is known as the photonic bandgap. A thorough overview of photonic crystals is given in reference [74].

Using these photonic crystals, accelerating waveguides can be designed that trap light in the bandgap of the crystal inside the guide. The light then becomes a propagating mode down the waveguide. Furthermore, as with metallic accelerating structures, by further tailoring the dielectric waveguide, a speed-of-light mode with a longitudinal electric field can be created that will accelerate electrons[75]. Figure 8.13 shows an example accelerating mode in a photonic crystal with a uniform longitudinal structure; called a photonic bandgap fiber $(\mathrm{PBF})[66,67]$. This plot was produced with the finite-difference time-domain (FDTD) method [76], using a freely available software package with subpixel smoothing for increased accuracy[77].

The potential use of hollow-core dielectric fibers as particle accelerators bring up many unique challenges that differ from typical telecommunications applications. Accelerating modes differ significantly from fundamental core modes in their field profiles, having the greatest field strengths just inside the inner most surface rather than in the center of the core. As a consequence, the accelerating modes are more easily perturbed by fabrication errors in the fiber than the core modes. Also, whereas telecomm applications are primarily concerned only in the transmission loss of a fiber, the application as an accelerator has stricter demands on the mode. In particular the mode must have a phase velocity within $\sim 0.01 \%$ of speed-of-light. This restriction is due to the large distance traveled by the beam during acceleration compared to the wavelength of the accelerating field. Typically, the accelerating modes in these structures have a group velocity of $0.3-0.5 \mathrm{c}$. Thus, for a $1 \mathrm{ps}$ long laser pulse, the electron beam will outrun the laser over a distance of $\sim 1 \mathrm{~mm}$. Over this distance, to maintain acceleration the phase velocity must precisely match the electron velocity such that the electrons slip relative to the laser field by only a small fraction of a wavelength, say $10 \%$ of $\lambda$. So the phase velocity must be controlled to within 


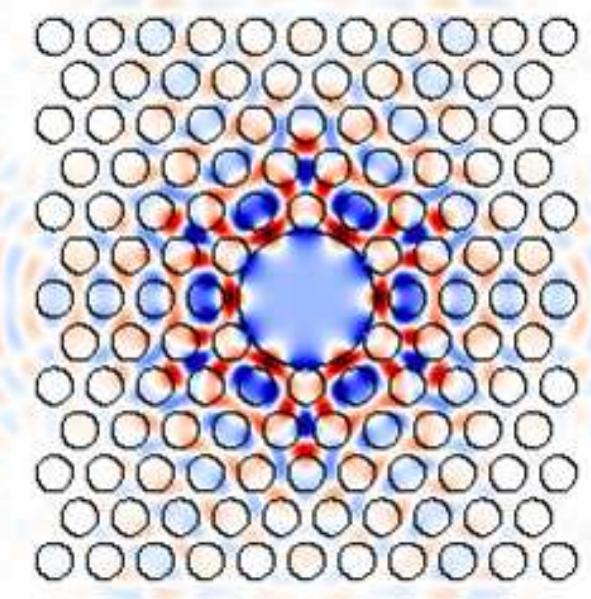

Figure 8.13: A cross-section showing an accelerating mode in a photonic band gap fiber. The mode propogates into the page; the color scale denotes the longitudinal electric field. The small holes have a radius of $0.35 \mu \mathrm{m}$, the large central hole $1.3 \mu \mathrm{m}$, and the accelerating freespace wavelength equal to $0.77 \mu \mathrm{m}$.

\section{$0.1 \times \lambda / 1 m m$ or $0.01 \%$.}

Unlike RF waveguides which can be diagnosed directly by bead pulls for example, direct examination of fiber fields is much more difficult. It therefore becomes interesting to consider probing candidate structures using the electron beam itself. The electron beam will excite modes of the structure that have a speed-oflight phase velocity and reasonable overlap with the beam's Cherenkov wake in the structure[78, 79, 80, 81]. Spectroscopy on the resulting excited modes will inform more precisely of the resonant frequencies of potential accelerating modes. Once potential SOL accelerating modes are identified, the fiber can later be excited from light generated by an optical parametric amplifier.

Before attempting such an experiment, we simulate the structure using the particlein-cell code Magic[82]. A short section of fiber is excited by a short electron pulse whose duration is less than the wavelengths of interest. In the simulations to follow, a pulse is emitted off of a conducting boundary and propogates down the fiber. 
Perfectly matched layer (PML) boundaries at the perimeter of the simulation absorb outgoing radiation. Various monitors within the simulation monitor $E_{z}(t)$ and the flux generated by the beam. Although the emission from the beam in the simulation is coherent, we can also use the results to calculate the amount of incoherently generated wake from a longer electron pulse in the actual experiment.

\subsubsection{Resonant Cherenkov Wakefield from a Bragg Fiber}

The first fiber examined is an azimuthally symmetric Bragg structure. The fiber consists of a multilayer dielectric with varying index of refraction. The layer thicknesses are chosen to determine the confined wavelength. The innermost layer has a unique thickness to give the confined mode a SOL phase velocity. A full prescription for choosing the layer thicknesses can be found in reference [65]. For the simulation the fiber was designed to have a $1.5 \mu \mathrm{m}$ accelerating mode and an inner hole radius of also $1.5 \mu \mathrm{m}$. It is worth noting that while fibers with this specific design do not yet exist, Bragg fibers have been fabricated for other purposes, both with a silica-air layering[83] and two types of dielectric[84].

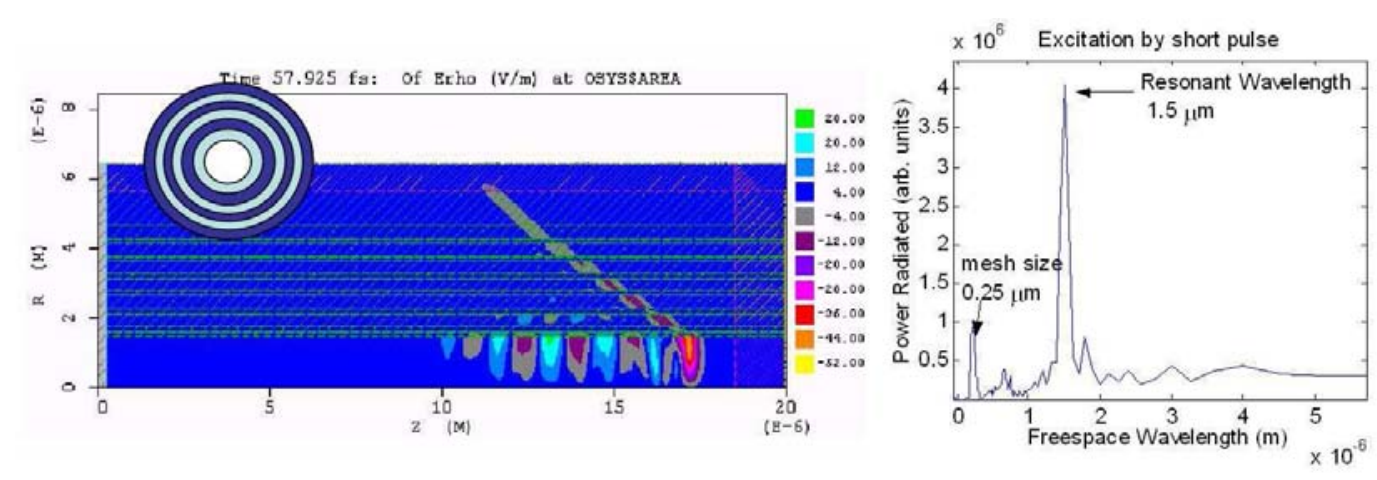

Figure 8.14: 2D simulation of the wake generated in a Bragg fiber. Left: Snapshot of $E_{r}$ in the fiber. Note the Cherenkov angle within the bulk dielectric and the wake generated. The inset shows the cross-section of the fiber. Right: Fourier transform of the on-axis $E_{z}$ field showing the resonance at the expected wavelength of $1.5 \mu \mathrm{m}$.

A simulation (figure 8.14) of a fiber Bragg structure shows the formation of a wake. The inset shows a cross-section of the fiber. On the right of figure 8.14 is an FFT of 
the on-axis $E_{z}$ field near the end of the fiber to give a sense of the resulting spectrum. The resonance is clear in both the simulation snap shot and resulting spectrum. The FFT also shows a simulation artifact resulting from an excitation at the grid spacing of $0.25 \mu \mathrm{m}$. Visible in the simulation snap-shot is both the formation of the resonant mode behind the bunch as well as a trailing field flux that radiates out of the sides of the simulation. This trailing edge is the Cherenkov wake that follows the electron beam and provides an additional loss mechanism to the beam energy besides the resonant mode excitation. Flux calculations in the simulation indicate a $50 \mathrm{pC}$ bunch traveling through a $1 \mathrm{~mm}$ fiber will produce $\sim 2 \mathrm{~nJ}$ of incoherent Cherenkov radiation.

We can calculate the energy radiated into the accelerating mode using the interaction impedance $Z_{\text {int }}$ (eqn. 8.1) of the structure. An electron passing through a structure will excite the accelerating mode, in the process losing energy. The energy loss per unit length, $d W / d z$, is given by[85]:

$$
\frac{d W}{d z}=\frac{q^{2}}{4} \frac{c \beta_{g}}{1-\beta_{g}} \frac{Z_{i n t}}{\lambda^{2}}
$$

where $\beta_{g}$ is the group velocity of the mode and $\lambda$ is the wavelength of the mode. The interaction impedance $Z_{\text {int }}$ and group velocity must be calculated for a particular structure. In this case for the cylindrical Bragg waveguide, it can be found analytically $[80,81]$. For the parameters used in the simulation, we have $Z_{\text {int }}=19 \Omega$ and $\beta_{g}=0.45$.

Although the simulation uses a short electron pulse to excite a coherent wake, an initial experiment will rely on an incoherent excitation of the accelerating mode (a.k.a. Schottky noise) from a long electron pulse. Coherent effects between particles average to zero. The total loss is then simply $W$ times the number of particles in the bunch. So, for a $50 \mathrm{pC}$ bunch and a $1 \mathrm{~mm}$ long fiber we expect an energy loss of $4.3 \mathrm{pJ}$. Although a small amount, this is still much greater than the coherent optical transition radiation yield in the microbunching experiment (§6) which produced $<100$ fJ at the fundamental. 


\subsubsection{The 3-D case: Crystal Fibre HC-1550-02 Fiber[1]}

While a custom fiber designed specifically for laser acceleration is being pursued, the appeal of using a commercially available fiber is obvious. The design and development as well as many of the diagnostic measurements are already done. Crystal Fibre currently has available a range of air core photonic bandgap fibers that may potentially be suitable as accelerator structures. For instance, the HC-1550-02 fiber designed to support an $H E_{11}$ fundamental mode at $1.55 \mu \mathrm{m}$ has also been found to support a SOL accelerating mode at $1.89 \mu \mathrm{m}[86]$.

The HC-1550 has an inner core of $10.9 \mu \mathrm{m}$, much larger than the wavelengths of the modes themselves. As a result, the waveguide is both highly overmoded and also less strongly coupled to the electron beam. The induced wakefield is less obvious in snap shots of the simulation (figure 8.15). The simulation approximates the HC-155002 as a set of perfect cylinders. As with the Bragg fiber, an examination of the $E_{z}$ field on axis shows excitation of modes in the near IR. For example we see excitation at $1.89 \mu \mathrm{m}$ where previous simulation with the code CUDOS found a SOL mode[86]. The simulation of the HC-1550 fiber remains an on-going effort in preparation for plans to test a real fiber with an electron beam.

As with the cylindrical Bragg fiber, we can calculate an expected yield in a Schottky noise wakefield experiment from the interaction impedance $Z_{\text {int }}$. Unlike the Bragg fiber, however, $Z_{\text {int }}$ cannot be calculated analytically, but can be estimated from field maps produced by eigenmode solvers for the fibers[86]. From these simulations, we obtain interaction impedance of $Z_{\text {int }}=0.2 \Omega$ and $\beta_{g}=0.7$ for the mode with wavelength $\lambda=1.89 \mu \mathrm{m}$. For the same $50 \mathrm{pC}$ beam in a $1 \mathrm{~mm}$ fiber we therefore expect a yield of $0.078 \mathrm{pJ}$. The much smaller yield is a direct result of the fiber having a much larger core diameter which in turn drops the interaction impedance.

\subsubsection{Potential Experimental Arrangement}

Although an experiment studying optical wakefields in a photonic bandgap fiber was unfortunately not possible within the time frame of the thesis, plans for the experiment have begun to take shape and can be tentatively described here. The key 

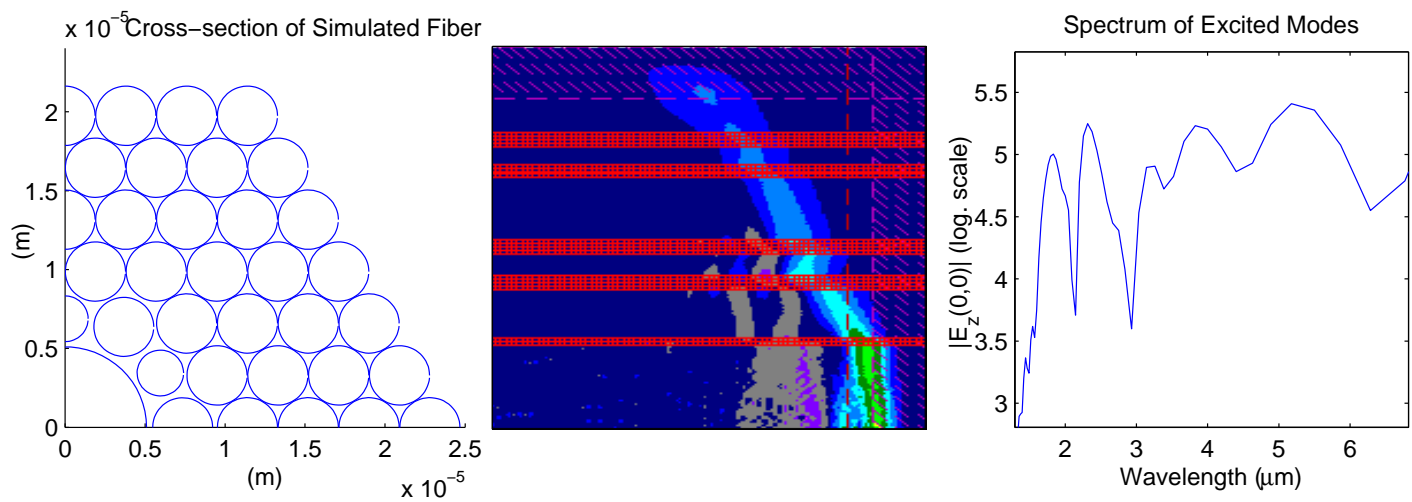

Figure 8.15: Left: Approximated cross-section of HC-1550 used in simulation. Center: Snap-shot of $E_{z}$ field produced by beam traversing fiber. Right: Resulting spectrum excited by the electron beam.

challenge in an experiment will be aligning the focused electron beam from the PMQ triplet into the fiber. The beam and fiber must be aligned to within fractions of a micron transversely, and the beam longitudinal focus located with a few hundred microns (recall from $\S 8.2$ that the electron focal depth is $\beta *=0.5 \mathrm{~mm}$ ). In addition, the beam to fiber angle must be controlled to keep the electron beam within the narrow fiber core over an appreciable distance of $1 \mathrm{~mm}$. The aperture of $2.5 \mu \mathrm{m}^{3}$. requires angular precision of $2.5 \mu \mathrm{m} / 1 \mathrm{~mm}=2.5 \mathrm{mrad}$ in each transverse dimension.

Figure 8.16 shows the layout of the fiber wakefield experiment. The PMQ triplet produces a focus $\sim 2 \mathrm{~cm}$ after the final quadropole face. An assembly including the fiber is positioned at the focus. The assembly will feature horizontal and vertical position control, and potentially tip/tilt control also. Along with the fiber, the assembly will include several diagnostics. A mirror will be used to generate incoherent transition radiation from the electron beam for observing the beam profile. A Ce:YAG crystal will also be present to use in the event that OTR yield is insufficient to observe the beam. The Ce:YAG spot will likely be dominated by blooming effects in the crystal, but could still be useful in extrapolating the centroid position of the electron beam or the aspect ratio. Finally, mounted on the assembly will be a $\sim 200 \mu \mathrm{m}$ thick tungsten rectangle with one corner exposed unsupported which will be used for knife

\footnotetext{
${ }^{3}$ The experiment will likely use a fiber with smaller dimensions. The reason for this is described further on in this section
} 


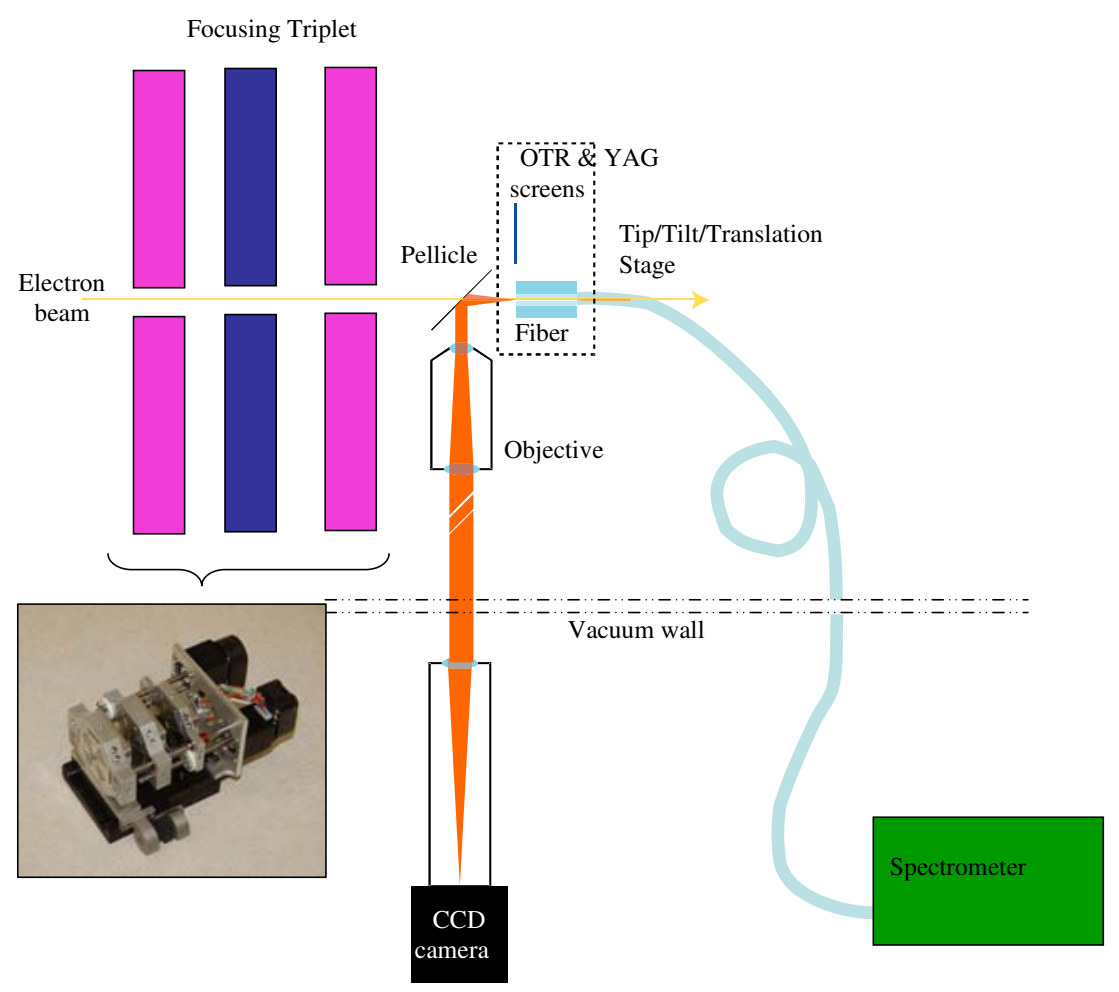

Figure 8.16: Schematic of the fiber wakefield experiment layout.

edge measurements of the beam transverse envelope.

The fiber is held straight for $1 \mathrm{~mm}$, the focal depth of the electron beam. For a first experiment the fiber is then bent quickly away from the beam with a bend radius of $4 \mathrm{~mm}$. This will mean that electrons will scatter through the glass of the fiber as the beam diverges from the focus. However, this will also greatly simplify directing the light generated in the first millimeter to diagnostics. The fiber will exit the vacuum through a fiber vacuum feedthrough and couple directly to a spectrograph. The spectrograph and its detectors are optimized for the visible, which makes studying the HC-1550-02 fiber difficult since simulations indicate the accelerating mode is out at $1.89 \mu \mathrm{m}$. The experiment will therefore use a smaller version of the PBG fiber made 
by Crystal Fibre called the HC-633-01 which simulations indicate has an accelerating mode in the near-IR, within range of the spectrograph. This brings with it the drawback of a smaller core diameter of only $5.1 \mu \mathrm{m}$. 


\section{Chapter 9}

\section{Conclusion}

This dissertation has presented several of the first steps in a research program looking to accelerate electrons directly with light. Magnetic hardware has been designed, fabricated, and used to produce an optically microbunched electron beam. This hardware, including a three period Hybrid-Halbach undulator and an electromagnetic chicane, was designed, fabricated, and measured in-house at SLAC. The designs included tunability and vacuum compatibility as the hardware was placed entirely in vacuum.

A series of experiments were performed using the microbunching hardware. The first experiments were aimed primarily at establishing an interaction between electron and laser with visible light. This first experiment (ch. 4), performed at the HEPLSCA facility, explored harmonics of the IFEL interaction. The experiment used only the undulator, and scanned the undulator gap to satisfy the FEL resonance condition for multiple harmonics. This experiment, together with the ITR experiment[25], was the first successful demonstration of a laser-electron interaction at $800 \mathrm{~nm}$ where the electrons traveled in vacuum.

The later experiments, performed at the newly commissioned E163 facility at SLAC (ch 5), produced and accelerated an optically microbunched beam. The first of these experiments characterized the microbunches using coherent optical transition radiation (COTR). The data showed good agreement with the analytic theory for bunch formation. In the second experiment the microbunch hardware was combined 
with the ITR accelerator serving as a second stage in a net acceleration experiment. The experiment succeeded in demonstrating net acceleration of electrons directly with visible light. Although the total acceleration was very modest, the insight gained will prove invaluable to future efforts which should demonstrate significant acceleration. Most notably, successful demonstration of net acceleration required precise measurement and control of the optical phase between the microbunches and the accelerating field.

In addition to the microbunching hardware, efforts were begun to tackle a second issue with optical acceleration: coupling the electron beam to small structures. Although the ITR and IFEL processes use large electron and laser spots, future acceleration schemes will pass the electron and laser through structures with optical scale dimensions. These small structures will have far superior acceleration gradients and efficiencies compared to the IFEL and ITR process, but present a unique challenge in passing the electron beam through the structure. The conventional focusing elements available in the experiment fall far short in producing the necessary focused electron spots to efficiently pass electron through the structures. Instead, a permanent magnet quadrupole (PMQ) triplet has been designed and fabricated. This device can produce much smaller electron spots than the conventional magnets. Although time for testing this device was not available within the time frame of the thesis, the PMQ triplet will soon be used in upcoming experiments.

\subsection{Future of the E163 Program}

The first experiment planned after the net acceleration experiment was outlined in section 8.4. This experiment will be the first use of the PMQ triplet and will both serve to characterize the performance of the triplet as well as be the first pass at observing the wakefield of an electron beam passing through an optical structure. Efforts are currently underway to design the experiment layout, select hardware, and simulate the expected response in the wakes.

During the first year after completing this thesis work, several experiments are 
planned as the first of the optical scale structures. If the wakefield experiment is successful in identifying an accelerating mode in an off-the-shelf fiber, an experiment will be designed to couple in laser power and show acceleration in the photonic bandgap fiber.

Other structures proposed include two planar structures. The first is a grating structure that illuminates the structure from the side with a free-space propagating laser mode. Instead of coupling power into an optical waveguide, the structure is designed to excite an evanescent field above the grating such that the electrons are accelerated. Remaining laser power continues to propagate transversely through the structure re-emerging as a free space mode on the other side.

The second planar structure is a Bragg waveguide[89] that confines the light in a speed-of-light accelerating mode that travels with the electrons. The structure includes a coupling mechanism for the laser light. As in the prior planar structure, light is incident transverse to the waveguide. In the first part of the waveguide a grating is placed on the inside of the waveguide such that power is sent into the guide[89]. Efforts to fabricate this structure are currently on-going.

A final structure currently under investigation is the woodpile structure. The structure consists (Fig. 9.1,c) of layers of rods with rectangular cross-sections, with each layer rotated through $90^{\circ}$ with respect to the prior layer. In addition, alternate layers are offset with respect to one another. This structure has been investigated as an accelerating structure by forming a waveguide region within the woodpile[88]. Work is now underway to fabricate the structure.

All of these structures have potential advantages and disadvantages. The photonic crystal fibers like the HC-1550-02 already exist and are in-hand. However, efficiently coupling light into an accelerating type mode has not yet been demonstrated. Even worse, despite extensive simulation efforts, it is still not clear whether any of these fibers actually support accelerating modes. The woodpile structure has the potential advantage in that due to its lithographic fabrication process, it might most readily couple to other lithographically produced structures including power sources, distribution, and phase control. However, fabricating an entire woodpile accelerator including the waveguide has not been completed and could potentially take several 


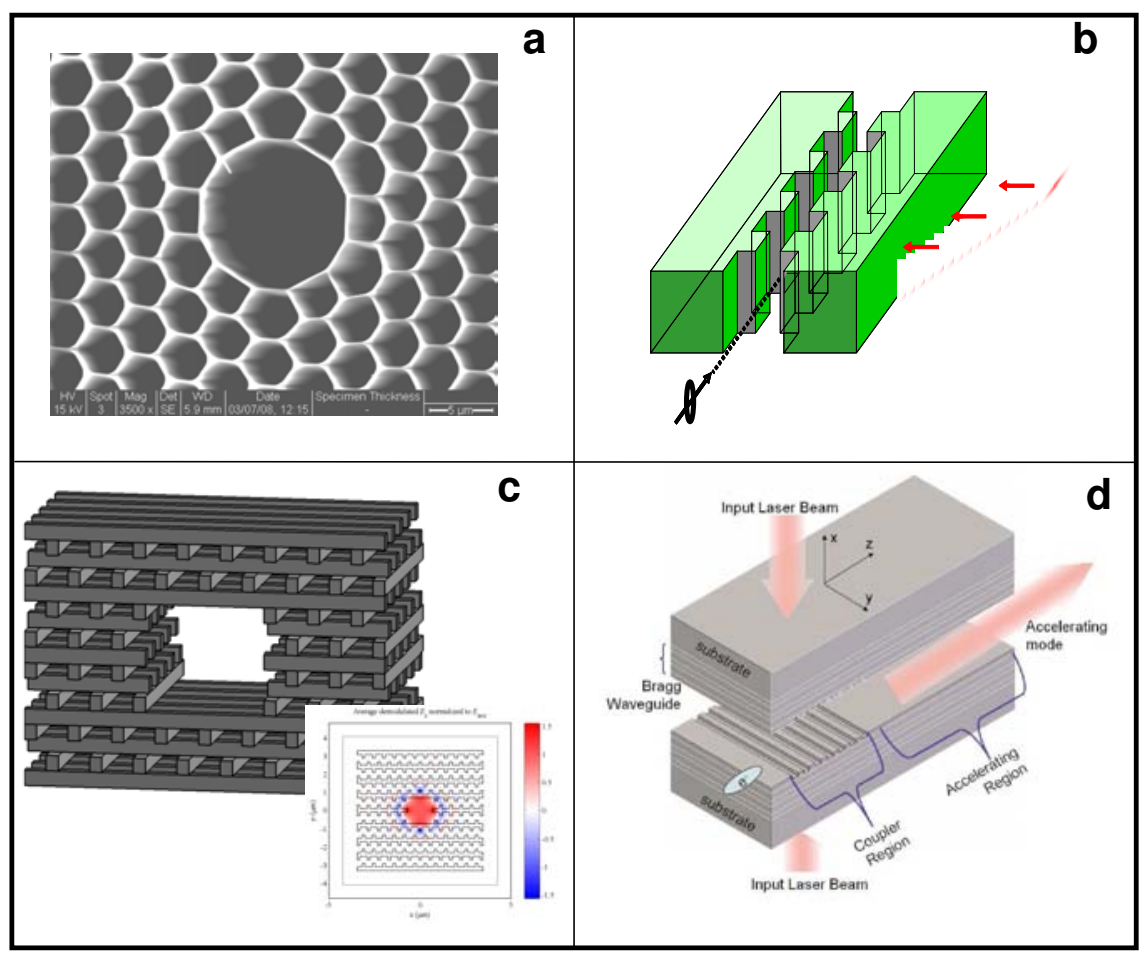

Figure 9.1: Optical scale accelerating structures currently being investigated. a) An SEM image of the Crystal Fibre HC-1550-02 fiber[1]. b) The grating evanescent field accelerator[87]. c) the 'woodpile' 3D photonic bandgap structure with waveguide defect. The inset shows the accelerating field in the waveguide[88]. d) The planar Bragg waveguide accelerator with a grating coupler region (figure reproduced from [89]).

years.

The two planar structures offer the best near-term chance of seeing electron beam tests. Both structures feature coupling to a free-space propagating laser beam. For the evanescent structure the key challenge will be alignment of the two halves. This step can be done ahead of electron experiments by passing a laser transversely through the structure and observing the interference pattern due to the gratings. 


\subsection{Potential Impact on the Broader Research Com- munity}

Beyond the immediate scope of direct laser acceleration and the E163 program, the research presented in this dissertation could find uses in many other applications in accelerator physics. Optical microbunching has been discussed and experimentally demonstrated for radiation production via High-Gain Harmonic Generation (HGHG) Free-Electron-Lasers[90]. In these devices, an IFEL is used to bunch an electron beam. This microbunched beam is then passed through another undulator whose period is chosen such that the resonance condition is satisfied at a higher harmonic of the original bunching wavelength. Some have proposed staging a series of such undulators in a cascade to reach a very high harmonic of the original bunching wavelength[91]. An HGHG FEL has the significant advantage of SASE FELs in that the final output inherits the coherence of the seed laser.

Several of the experimental techniques demonstrated in the IFEL microbunching may find use in SASE FELs in the form of atto-slicing of an electron beam. In this arrangement, an IFEL is used in conjunction with a few optical cycle laser pulse to form one or a few microbunches within an longer electron pulse. The microbunch has a higher peak current than the surrounding charge in the beam, and therefore reaches saturation in a second FEL sooner allowing for a shorter undulator. This has been termed, enhanced SASE, or ESASE[92]. With the parameters chosen correctly a single X-ray pulse can be generated with a pulse length $<1$ fs.

Besides light sources, microbunched electron beams could be used in more exotic accelerator schemes. For instance, a recent experiment used a microbunched beam to drive a wakefield in a plasma[93]. In this experiment, the microbunched beam was formed by passing an energy-chirped, energy-dispersed beam through a grating. This formed a pair of bunches that were both energy and time separated. The spacing was $\sim 0.5$ ps. An IFEL based microbuncher could produce microbunches with much closer spacing. 
The research presented in this dissertation marks the beginning of a very ambitious research program hoping to design novel accelerator structures operating in a regime unlike anything done before. There are many challenges to be overcome in designing optical scale accelerators, and many more likely to be uncovered. Several of the proposed structures lack a clear way to couple light into the structure. Wakefields and non-accelerating modes of the structure may perturb the beam and undermine the acceleration. Fabrication errors of the structure may scatter laser power away from the accelerating mode, shift the phase velocity of the mode away from synchronicity with the electron beam, or cause increased peak fields in the structure that will limit the maximum accelerating gradient before damage.

Even if an optical accelerating structure is demonstrated, the reality of an all optical accelerator would still be a far off promise. Steering and focusing magnets, beam monitors, power sources and phase control would all need to be reinvented on the optical scale in order to build an accelerator-on-a-chip. Although these challenges are outside the scope of a single research group, there are nevertheless issues to keep in mind in this product oriented research field.

Despite the challenges faced, the potential benefits of optical scale laser accelerators make for an intriguing research pursuit. The low per pulse energy needed to power an optical accelerator immediately lends itself to high repetition rate oscillators. Although wakefields limit the per pulse charge to just a few fC[94], the average current could be made up with the high repetition power source. The electron beam from an optical accelerator would have, by necessity of traveling down a very small micron scale tunnel, a very small emittance, orders of magnitude smaller than the current state-of-the art. This could allow for operation of novel light sources despite the low charge[95].

The next few years of the E163 program will hopefully see great success in the pursuit of all-optical accelerators. It is a promising research project with many diverse challenges to pursue. The small, initial steps taking in this dissertation should lay a useful groundwork upon which this future work may progress. 


\section{Appendix A}

\section{Multipole Expansion of Magnetostatic Fields}

For the field inside a magnet far from the edges such that we can ignore any small longitudinal field, we can write the two transverse field components as a single complex quantity with the real component being the $\mathrm{x}$-component of the magnetic field and the imaginary component being the y-component of the field. The complex field $B_{x}+i B_{y}$ can then be written as an expansion over the complex position $x+i y$ with complex coefficients.

$$
B_{x}+i B_{y} \equiv \sum_{n=1}^{\infty} \tilde{D}_{n}(x+i y)^{n-1}
$$

To determine the values of the complex coefficients $\tilde{D}_{n}$ we consider $N$ samples on a circle of radius $R$. We can the write:

$$
\begin{aligned}
x+i y & =\xi_{j}=R e^{\frac{2 \pi(j-1) i}{N}}=R e^{i \theta_{j}} \\
B_{x}\left(\xi_{j}\right)+i B_{y}\left(\xi_{j}\right) & =\sum_{n=1}^{\infty} \tilde{D}_{n} R^{n-1} e^{\frac{-2 \pi i}{N}(n-1)}
\end{aligned}
$$


130 APPENDIX A. MULTIPOLE EXPANSION OF MAGNETOSTATIC FIELDS

We can now note that:

$$
\sum_{j=1}^{N}\left[B_{x}\left(\xi_{j}\right)+i B_{y}\left(\xi_{j}\right)\right] e^{-i \theta_{j}\left(n^{\prime}-1\right)}=\sum_{j=1}^{N} \sum_{n=1}^{\infty} \tilde{D}_{n} R^{n-1} e^{\frac{2 \pi i}{N}(j-1)\left(n-n^{\prime}\right)}
$$

The right-hand-side equals zero unless $n=n^{\prime}$; therefore:

$$
\tilde{D}_{n}=\sum_{j=1}^{N} \frac{\left[B_{x}\left(\xi_{j}\right)+i B_{y}\left(\xi_{j}\right)\right]}{R^{n-1} N} e^{-i \theta_{j}(n-1)}
$$

Finally, we separate the real and imaginary parts of $\tilde{D}_{n} ; \tilde{D}_{n}=b_{n}+i a_{n}$ where $b_{n}$ is termed the "normal" component of the field and $a_{n}$ is called the "skew" term. 


\section{Appendix B}

\section{Bootstrap Error Analysis}

This appendix contains an extended description of the Bootstrap error analysis technique used in this dissertation. A full description of the Bootstrap technique can be found in an number of mathematical analysis textbooks, for instance: Davison, A.C. and Hinkley, D., "Bootstrap methods and their Application" [62].

The boot-strap technique is used for obtaining error estimates on a data analysis when the per point variance is unknown. In the case in chapter 6 , the analysis was a curve fit of a Gaussian to the IFEL cross-correlation data. The bootstrap technique takes several random subsets of the data set and performs the same analysis on each subset of the data. Error estimates are drawn from the spread in values of the results from each analysis.

For the analysis contained here-in, the simplest form of the Bootstrap technique is used. Each subset is a random selection of roughly $50 \%$ of the original dataset. There is no resampling or fake data generation using added noise. Thus, the subsets are smaller than the initial set, likely leading to an overestimate of the error.

For the data presented in figure 6.6, each data set was sub-sampled 100 times. The initial guess for each subset was also varied by $10 \%$ on each guess parameter. The error estimate was then set equal to \pm 1 standard deviation of the parameter distribution. This is equivalent to a confidence interval of $68 \%$.

Although the technique used herein may fall short of the methods described in more rigorous Bootstrap method texts, the error estimates generated do seem to 
give a reliable estimate when compared to the raw data; if anything overestimating. Perhaps more importantly, as mentioned in the text of chapter 6, the run-to-run variation turned out to be dominated more by systematic drifts than the individual run variations quantized by the Bootstrap error analysis. 


\section{Bibliography}

[1] http://www.crystal-fibre.com/datasheets/HC-1550-02.pdf.

[2] R.J. Van de Graaf. A 1,500,000 volt electrostatic generator. Physical Review, (38):1919, 1931.

[3] R. Wideröe. Archiv Elektrotechnik, 21:387, 1928.

[4] R. Brinkmann, K. Flöttmann, J. Robach, P. Schmüser, N. Walker, and H. Weise. Technical design report, part ii: The accelerator.

[5] D. Strickland and G. Mourou. Optical Communications, (56):219, 1985.

[6] David Pritzkau. RF Pulsed Heating. PhD thesis, Stanford University. Dept. of Applied Physics., 2001.

[7] http://www.ireap.umd.edu/High-Gradient

[8] J.D. Lawson. Lasers and accelerators. IEEE Transactions on Nuclear Science, NS-26:4217-4219, 1979.

[9] W. D. Kimura, A. van Steenbergen, M. Babzien, I. Ben-Zvi, L. P. Campbell, D. B. Cline, C. E. Dilley, J. C. Gallardo, S. C. Gottschalk, P. He, K. P. Kusche, Y. Liu, R. H. Pantell, I. V. Pogorelsky, D. C. Quimby, J. Skaritka, L. C. Steinhauer, and V. Yakimenko. First staging of two laser accelerators. Phys. Rev. Lett., 86(18):4041-4043, Apr 2001. 
[10] W.D. Kimura, L.P. Campbell, C.E. Dilley, S.C. Gottschalk, D.C. Quimby, A. van Steenbergen, M. Babzien, I. Ben-Zvi, J.C Gallardo, and K.P. Kusche et al. Detailed experimental results for laser acceleration staging. Physical Review Special Topics-Accelerators and Beams, 4(10):101301, 2001.

[11] P. Musumeci et al. High energy gain of trapped electrons in a tapered, diffractiondominated inverse-free-electron laser. Physical Review Letters, 94(15):154801/14, 2005.

[12] I.E. Piestrup et al. Momentum modulation of a free electron beam with a laser. Journal of Applied Physics, 46(1):132-7, 1974.

[13] W. Kimura et al. Laser acceleration of relativistic electrons with the inverse cherenkov effect. Physical Review Letters, 74(4):546-549, 1995.

[14] G. V. Stupakov and M. S. Zolotorev. Ponderomotive laser acceleration and focusing in vacuum for generation of attosecond electron bunches. Phys. Rev. Lett., 86(23):5274-5277, Jun 2001.

[15] D. Zheng and R.L. Byer. Proposed waveguide structure for laser driven electron acceleration. Proceedings of Advanced Accelerator Concepts 6th Workshop, Fontana WI., pages 156-162, 1994.

[16] M. Lenzner, J. Krüger, S. Sartania, Z. Cheng, Ch. Spielmann, G. Mourou, W. Kautek, and F. Krausz. Femtosecond optical breakdown in dielectrics. Phys. Rev. Lett., 80(18):4076-4079, May 1998.

[17] Jorg Kruger, Daniela Dufft, Robert Koter, and Andreas Hertwig. Femtosecond laser-induced damage of gold films. Applied Surface Science, 253(19):7815-19, 2007.

[18] A. P. Joglekar, H. Liu, G. J. Spooner, E. Meyhofer, G. Mourou, and A. J. Hunt. A study of the deterministic character of optical damage by femtosecond laser pulses and applications to nanomachining. Applied Physics B, 77:25, 2003. 
[19] P. Klopp, V. Petrov, U. Griebner, K. Petermann, V. Peters, and G. Erbert. Highly efficient mode-locked yb:sc2o3 laser. Optics Letters, 29(4):391-3, 2004.

[20] Reto Häring, Rüdiger Paschotta, Alex Aschwanden, Emilio Gini, Francois Morier-Genoud, and Ursula Keller. High-power passively mode-locked semiconductor lasers. IEEE JOURNAL OF QUANTUM ELECTRONICS, 38(9), 2002.

[21] K. Shimoda. Applied Optics, 1(33), 1962.

[22] T. Tajima and J. M. Dawson. Laser electron accelerator. Phys. Rev. Lett., 43(4):267-270, Jul 1979.

[23] Cameron G. Geddes. Plasma Channel Guided Laser Wakefield Accelerator. PhD thesis, University of California, Berkeley, 2002.

[24] K. Matsukado, T. Esirkepov, K. Kinoshita, H. Daido, T. Utsumi, Z. Li, A. Fukumi, Y. Hayashi, S. Orimo, M. Nishiuchi, S. V. Bulanov, T. Tajima, A. Noda, Y. Iwashita, T. Shirai, T. Takeuchi, S. Nakamura, A. Yamazaki, M. Ikegami, T. Mihara, A. Morita, M. Uesaka, K. Yoshii, T. Watanabe, T. Hosokai, A. Zhidkov, and A. Ogata. Energetic protons from a few-micron metallic foil evaporated by an intense laser pulse. Phys. Rev. Lett., 91(21):215001, Nov 2003.

[25] Tomas Plettner. Proof-of-principle experiment for crossed laser beam electron acceleration in a dielectric loaded vacuum structure. PhD thesis, Stanford University. Dept. of Applied Physics., 2002.

[26] T. Plettner, R. L. Byer, E. Colby, B. Cowan, C. M. S. Sears, J. E. Spencer, and R. H. Siemann. Visible-laser acceleration of relativistic electrons in a semi-infinite vacuum. Physical Review Letters, 95(13):134801, 2005.

[27] Christopher M. S. Sears, Eric R. Colby, Benjamin M. Cowan, Robert H. Siemann, James E. Spencer, Robert L. Byer, and Tomas Plettner. High-harmonic inversefree-electron-laser interaction at 800 nm. Physical Review Letters, 95(19):194801, 2005. 
[28] T. Plettner, R. L. Byer, E. Colby, B. Cowan, C. M. S. Sears, J. E. Spencer, and R. H. Siemann. Proof-of-principle experiment for laser-driven acceleration of relativistic electrons in a semi-infinite vacuum. Phys. Rev. ST Accel. Beams, 8(12):121301, Dec 2005.

[29] D. A. G. Deacon, L. R. Elias, J. M. J. Madey, G. J. Ramian, H. A. Schwettman, and T. I. Smith. First operation of a free-electron laser. Phys. Rev. Lett., 38(16):892-894, Apr 1977.

[30] A.S. Artamonov et al. The first experiments with an optical klystron installed on the vepp-3 storage ring. Nuclear Instruments and Methods in Physics Research, (177.1):247-52, 1980.

[31] Russell H. Varian and Sigurd F. Varian. A high frequency oscillator and amplifier. J. Appl. Phys., 10(5):321-327, 1939.

[32] R.M. Phillips. IRE Trans. Elec. Dev., (7):231, 1960.

[33] John M. J. Madey. J. Appl. Phys., (42):1906, 1971.

[34] Charles Brau. Free Electron Lasers. Academic Press, Boston, USA, 1990.

[35] W.B. Colson, G. Dattoli, and F. Ciocci. Phys. Rev. A, 31(2):828, 1985.

[36] I.S. Gradshteyn and I.M. Ryzhik. Tables of Integrals, Series, and Products, 6th ed. Academic Press, San Diego, CA, 6th edition, 2000.

[37] P. Musumeci et al. Phys. Rev. E, 72(1):16501, 2005.

[38] P.L. Webster. J. Appl. Phys., 10:501, 1939.

[39] S. Baccaro, F. De Martini, and A. Ghigo. Charge density harmonics generation in free-electron relativistic parametric devices. Opt. Lett., 7(4):174, 1982.

[40] A. Luccio, G. Matone, L. Miceli, and G. Giordano. Laser and Particle Beams, 8(3):383-398, 1990. 
[41] L.C. Steinbauer and W.D. Kimura. Longitudinal space charge debunching and compensation in high-frequency accelerators. Physical Review Special TopicsAccelerators and Beams, 2(8), 1999.

[42] S. Reiche. Genesis 1.3: a fully 3d time-dependent fel simulation code. Nucl. Instrum. Methods Phys. Res. A, 429:243248, 1999.

[43] Michael Borland. Computer code: Elegant. http://www.aps.anl.gov/asd/oag/oaghome.shtml.

[44] B. Brown, K. Halbach, J. Harris, and H. Winick. Nuclear Instruments and Methods in Physics Research, 208(1-3):65-77, 15 April 1983. Conference: X-Ray and VUV Synchrotron Radiat Instrum, Proc of the Int Conf; Aug 9-13 1982; Hamburg, W Ger.

[45] R. Klatt et al. Mafia - A 3D Electromagnetic CAD System for Magnets, RFStructures and Transient Wakefield Calculations, 1986.

[46] O. Chubar, P. Elleaume, and J. Chavanne. Journal of Synchrotron Radiation, 5:481-4, 1998.

[47] http://www.ugs.com/products/velocity/solidedge/solidEdge.shtml.

[48] C. McGuinness et al. Timing stability and control at the e163 laser acceleration experiment. Proceedings of PAC 200\%, Albequerque, New Mexico, USA, 2007.

[49] J. Hebling. Optical and Quantum Electronics, 28:1759-1763, 1996.

[50] J. Hebling. Derivation of the pulse front tilt caused by angular dispersion. Optical and Quantum Electronics, 28(12):1759-1763, 1996.

[51] Dennis Thomas Palmer. The Next Generation Photoinjector. PhD thesis, Stanford University, 1998.

[52] Eric Colby. PhD thesis, UCLA, 1997. FERMILAB-THESIS-1997-03. 
[53] D.T. Palmer et al. Proceedings of Particle Accelerator Conference, page 2251, 2001.

[54] L. Serafini and J.B. Rosenzweig. Physical Review E, 55:7565, 1997.

[55] P. B. Wilson, Z. D. Farkas, and R. D. Ruth. Sled ii: A new method of rf pulse compression. Linear Accelerator Conference, Albuquerque, NM, 1990. SLACPUB-5330.

[56] V. Yakimenko, M. Babzien, I. Ben-Zvi, R. Malone, and X.-J. Wang. Electron beam phase-space measurement using a high-precision tomography technique. Phys. Rev. ST Accel. Beams, 6(12):122801, Dec 2003.

[57] http://www.piacton.com/.

[58] L.A Vardanyan, G.M. Garibian, and C. Yang. Izv. Acad. Nauk Arm. SSR Fiz, 10:350, 1975 .

[59] V.L. Ginsburg and I.M. Frank. JETP (USSR), 16(15), 1946.

[60] J. Rosenzweig, G. Travish, and A. Tremaine. Coherent transition radiation diagnosis of electron beam microbunching. Nuclear Instruments and Methods in Physics Research A, 365:255-259, 1995.

[61] Max B. Reid. J. Appl. Phys., 70(11), 1991.

[62] A.C. Davison and D. Hinkley. Bootstrap methods and their Application. Cambridge University Press, 1997.

[63] C. M. S. Sears, R. L. Byer, E. Colby, B. M. Cowan, R. Ischebeck, C. McGuinness, R. J. Noble, T. Plettner, R. H. Siemann, J. Spencer, and D. R. Walz. Optical wakefield from a photonic bandgap fiber accelerator. In Proceedings of PAC 2007, Albuquerque, New Mexico, USA, page 3106, 2007.

[64] T. J. Kane and R. L. Byer. Monolithic, unidirectional single-mode ring laser. Opt. Lett., 10(2):65, 1985. 
[65] A. Mizrahi and L. Schachter. Optical bragg accelerators. Physical Review E, 70(1):016505, 2004.

[66] B. Cowan, M. Javanmard, and R.H. Siemann. Photonic crystal laser accelerator structures. Particle Accelerator Conference, 12-16 May 2003, Portland, OR, USA, 3:1855-7, 2003.

[67] B. Cowan. Two-dimensional photonic crystal accelerator structures. Physical Review Special Topics: Accelerators and Beams, 6(101301), 2003.

[68] Stanley Humphries Jr. Principles of Charged Particle Acceleration. John Wiley and Sons, 1986.

[69] Stanley Humphries Jr. Charged Particle Beams. John Wiley and Sons, 1990.

[70] J. B. Rosenzweig and P. Chen. Phys. Rev. D, 39(2039), 1989.

[71] J. Lim et al. Physical Review Special Topics: Accelerators and Beams, 8(072401), 2005.

[72] T. Mihara, Y. Iwashita, M. Kumada, E. Antokhin, and C.M. Spencer. A super strong adjustable permanent magnet for the final focus quadrupole in a linear collider. Proceedings of EPAC 2004, Lucerne, Switzerland, pages 1708-1710, 2004.

[73] R.W. Warren. NIM A, A272:257-263, 1988.

[74] John D. Joannopoulos, Robert D. Meade, and Joshua N. Winn. Photonic Crystals: Molding the Flow of Light. Princeton University Press, 41 William St., Princeton, New Jersey, 08540, 1995.

[75] Xintian Eddie Lin. Photonic band gap fiber accelerator. Phys. Rev. ST Accel. Beams, 4(5):051301, May 2001.

[76] Allen Taflove and Susan C. Hagness. Computational Electrodynamics: The Finite-Difference Time-Domain Method. Artech, Norwood, MA, 2000. 
[77] Ardavan Farjadpour, David Roundy, Alejandro Rodriguez, Mihai Ibanescu, Peter Bermel, J. D. Joannopoulos, Steven G. Johnson, and Geoffrey Burr. Improving accuracy by subpixel smoothing in fdtd. Optics Letters, 31(20):2972-2974, 2006.

[78] Chiyan Luo, Mihai Ibanescu, Steven G. Johnson, and J. D. Joannopoulos. Cerenkov radiation in photonic crystals. Science, 299(5606):368-371, 2003.

[79] N. Zabala, F.J.Garcia de Abajo, A. Rivacoba, A.G. Pattantyus-Abraham, M.O. Wolf, and L.A. Blanco P.M. Echenique. Stopping power and cherenkov radiation in photonic crystals. Nuclear Instruments and Methods in Physics Research, Section B, 230(1-4):24-30, 2005.

[80] L. Schächter. Wake field in dielectric acceleration structures. Physical Review E, 68(036502), 2003.

[81] L. Schächter. Erratum: Wake field in dielectric acceleration structures [ phys. rev. e 68, 036502 (2003) ]. Phys. Rev. E, 69(4):049901, Apr 2004.

[82] User-configurable magic for electromagnetic pic calculations. Computer Physics Communications, 78:54-86, 1995. Magic is a commercially produced code maintained by the Mission Research Corporation, http://www.mrcwdc.com.

[83] C. Jakobsen H.J. Deyerl T.P. Hansen B.H. Larsen J.B. Jensen T. Sorensen M. Terrel Y. Huang et. al. G. Vienne, Y. Xu. OSA Trends in Optics and Photonics Series, 95:715-717, 2004.

[84] S. Fan C. Chen J.D. Joannopoulos Y. Fink, D.J. Ripin and E.L. Thomas. J. Lightwave Technol., 17:2039-2041, 1999.

[85] K. L. F. Bane and G. Stupakov. Impedance of a rectangular beam tube with small corrugations. Phys. Rev. ST Accel. Beams, 6(2):024401, Feb 2003.

[86] R. Noble, E.R. Colby, B. Cowan, C.M.S. Sears, R.H. Siemann, and J.E. Spencer. Designing photonic bandgap fibers for particle acceleration. Proceedings of the IEEE Particle Accelerator Conference; 2007, pages 3103-3105, 2007. 
[87] T. Plettner, P. P. Lu, and R. L. Byer. Proposed few-optical cycle laser-driven particle accelerator structure. Phys. Rev. ST Accel. Beams, 9(11):111301, Nov 2006.

[88] Benjamin M. Cowan. Three-dimensional dielectric photonic crystal structures for laser-driven acceleration. Phys. Rev. ST Accel. Beams, 11(1):011301, Jan 2008.

[89] Zhiyu Zhang, Sami G. Tantawi, and Ronald D. Ruth. Distributed gratingassisted coupler for optical all-dielectric electron accelerator. Phys. Rev. ST Accel. Beams, 8(7):071302, Jul 2005.

[90] L. H. Yu, L. DiMauro, A. Doyuran, W. S. Graves, E. D. Johnson, R. Heese, S. Krinsky, H. Loos, J. B. Murphy, G. Rakowsky, J. Rose, T. Shaftan, B. Sheehy, J. Skaritka, X. J. Wang, and Z. Wu. First ultraviolet high-gain harmonicgeneration free-electron laser. Phys. Rev. Lett., 91(7):074801, Aug 2003.

[91] L. H. Yu et al. Nuclear Instruments and Methods A, 318:726, 1992.

[92] Alexander A. Zholents. Method of an enhanced self-amplified spontaneous emission for x-ray free electron lasers. Phys. Rev. ST Accel. Beams, 8(4):040701, Apr 2005.

[93] Efthymios Kallos, Tom Katsouleas, Wayne D. Kimura, Karl Kusche, Patric Muggli, Igor Pavlishin, Igor Pogorelsky, Daniil Stolyarov, and Vitaly Yakimenko. High-gradient plasma-wakefield acceleration with two subpicosecond electron bunches. Physical Review Letters, 100(7):074802, 2008.

[94] Y. C. Neil Na, R. H. Siemann, and R. L. Byer. Energy efficiency of an intracavity coupled, laser-driven linear accelerator pumped by an external laser. Phys. Rev. ST Accel. Beams, 8(3):031301, Mar 2005.

[95] T. Plettner and R. L. Byer. Proposed dielectric-based microstructure laser-driven undulator. Phys. Rev. ST Accel. Beams, 11(3):030704, Mar 2008. 